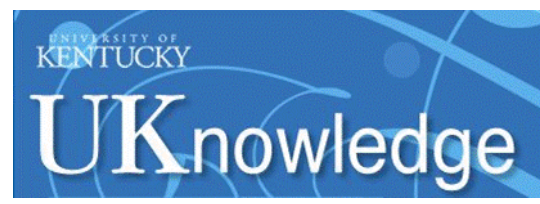

University of Kentucky

UKnowledge

\title{
FURTHERING PHARMACOLOGICAL AND PHYSIOLOGICAL ASSESSMENT OF THE GLUTAMATERGIC RECEPTORS AT THE DROSOPHILA NEUROMUSCULAR JUNCTION
}

Junyoung Lee

University of Kentucky, c14586@gmail.com

Right click to open a feedback form in a new tab to let us know how this document benefits you.

\author{
Recommended Citation \\ Lee, Junyoung, "FURTHERING PHARMACOLOGICAL AND PHYSIOLOGICAL ASSESSMENT OF THE \\ GLUTAMATERGIC RECEPTORS AT THE DROSOPHILA NEUROMUSCULAR JUNCTION" (2009). University \\ of Kentucky Master's Theses. 619. \\ https://uknowledge.uky.edu/gradschool_theses/619
}

This Thesis is brought to you for free and open access by the Graduate School at UKnowledge. It has been accepted for inclusion in University of Kentucky Master's Theses by an authorized administrator of UKnowledge. For more information, please contact UKnowledge@lsv.uky.edu. 
ABSTRACT OF THESIS

\section{FURTHERING PHARMACOLOGICAL AND PHYSIOLOGICAL ASSESSMENT OF THE GLUTAMATERGIC RECEPTORS AT THE DROSOPHILA NEUROMUSCULAR JUNCTION}

Drosophila larval neuromuscular junctions (NMJs) serve as a model for synaptic physiology. The molecular sequence of the postsynaptic glutamate receptors has been described; however, the pharmacological profile has not been fully elucidated. Despite the postsynaptic molecular sequence used to classify the receptors as a kainate subtype, they do not respond pharmacologically as such. Kainate does not depolarize the muscle, but dampens evoked EPSP amplitudes. Quantal responses show a decreased amplitude and area under the voltage curve indicative of reduced postsynaptic receptor sensitivity to glutamate transmission. ATPA, a kainate receptor agonist, did not mimic kainate's action. The metabotropic glutamate receptor agonist t-ACPD had no effect. Domoic acid, a quisqualate receptor antagonist, blocks the postsynaptic receptors without depolarizing the muscle, which supports the presence of quisqualate subtype receptors. The results suggest a direct postsynaptic action of kainate due to partial antagonist action on the quisqualate receptors. There does not appear to be presynaptic auto-regulation via a kainate receptor subtype or a metabotropic 
auto-receptor. A complete pharmacological profiling of the known receptor subtypes at this NMJ has not yet occurred; however, this study aids in furthering the ongoing investigations to provide a clearer picture of pharmokinetic profile and specificity of the receptor subtypes.

KEY WORDS: Synaptic facilitation, NCX, calcium, Drosophila larval heart

Junyoung Lee

August 2, 2009 
FURTHERING PHARMACOLOGICAL AND PHYSIOLOGICAL ASSESSMENT OF THE GLUTAMATERGIC RECEPTORS AT THE DROSOPHILA NEUROMUSCULAR JUNCTION

By

Junyoung Lee

Dr. Robin L. Cooper

Director or Thesis

Dr. Brian C. Rymond

Director of Graduate Studies

August 2, 2009 


\section{RULES FOR THE USE OF THESES}

Unpublished theses submitted for the Master's degree and deposited in the University of Kentucky Library are as a rule open for inspection, but are to be used only with due regard to the rights of the authors.

Bibliographical references may be noted, but quotations or summaries of parts may be published only with the permission of the author, and with the usual scholarly acknowledgments.

Extensive copying or publication of the thesis in whole or in part also requires the consent of the Dean of the Graduate School of the University of Kentucky.

A library that borrows this thesis for use by its patrons is expected to secure the signature of each user. 
Thesis

Junyoung Lee

The Graduate School

University of Kentucky

2009 


\title{
FURTHERING PHARMACOLOGICAL AND PHYSIOLOGICAL ASSESSMENT OF THE GLUTAMATERGIC RECEPTORS AT THE DROSOPHILA NEUROMUSCULAR JUNCTION
}

\author{
THESIS \\ A thesis submitted in partial fulfillment of the \\ requirements for the degree of Master in the \\ College of Arts and Sciences at the \\ University of Kentucky \\ By \\ Junyoung Lee \\ Lexington, Kentucky \\ Director: Dr. Robin Lewis Cooper, Associate Professor \\ 2009
}

Copyright @ Junyoung Lee 2009 


\section{ACKNOWLEDGMENTS}

I came to the University of Kentucky from South Korea two years ago. I am in active duty as a captain in the Korean Army. I was sent here by the Korean Army to learn Biology and experimental methods to address how the nervous system functions. When I began pursuing this masters program, I had little knowledge of the biological field. When I was a student at the Korean Military Academy I focused on Chemistry and Military Science. The time I spent in the biology department was stressful and compounded with frustration, as the language was new to me and the courses moved along very rapidly. So I feel extremely happy and lucky to have made it through the program. It was not a miracle that I survived in this foreign country, as I had very helpful people around me. It is a great pleasure to have a chance to be aquatinted with them.

The first person I appreciate is Dr. Robin L Cooper who is my mentor. I emphatically state that he is one of the best professors I have experienced. He has helped me a lot to feel comfortable both inside and outside the lab. His patience with me was nothing short of miraculous and he even never lost smile on his face. 설레임과 두려움을 안고 미국으로 온 저에게 항상 웃는 얼굴로 학업 및 생활하는데 불편함이 없도록 직접 보살펴 주시고, 많이 바쁜 와중에도 저의 엉뚱한 질문도 그냥 넘기는 것 없이 자상하게 대해주시는 모습을 보면서 남몰래 눈물을 흘린 적도 있었습니다. In fact, it is impossible for me to relay of all gratitude and respect in this letter. 이 곳 렉싱턴에서의 교수님과 함께했던 시간은 소중한 추억으로 간직되어 평생 잊지 못할 것입니다. 쿠퍼 교수님 그리고 사모님 감사합니다.

I appreciate my committee members, Dr. Doug Harrison and Dr. Bruce O'Hara for their expertise and intellectual conversations over the years.

I also acknowledge all professors at the chemistry department in Korea Military Academy. They always led cadets and their juniors by taking the initiative. What I had seen and learned from them became the cornerstones of my life and the motives of studying here. Among them, I should especially thank Dr. IL-Woo Yang, Dr. Nam-Taek Lee and Dr. Woo-Young Chung for willingly writing letters of recommendation and for encouragement to me. Also, I could not have been happier when I learned that Dr. Woo-Young Chung and his family were spending a sabbatical year in Lexington, KY. 지금도 공부를 하다가 ‘교수님이 안 계셨으면 어떻게 되었을까?’하고 생각을 하곤 합니다. 표현이 서툴러 교수님께 감사하다는 말씀도 못드렸습니다. 제가 느끼고 배운 것들을 소중히 간직하여 베풀고 실천하면서 살도록 항상 노력하겠습니다. 감사합니다. 
I had a pleasurable time with my lab colleagues, Sonya Bierbower and Mohati Desai. I can surely say you guys are my family. You guys helped me a lot to get through the hard times and to become familiar to the American society. Time being with you guys was always awesome. I should not forget a lot of new words I learned from Sonya. Also, I appreciate many other graduate and undergraduate students that worked in the lab over the past 2 years.

Appreciation is expressed to Korean Army Headquarters that supported my tuition and fees. I did not have to worry about financial matters while studying. Their support was a great advantage to me to focus on research and curriculum. 마지막으로, 제 인생에서 가장 중요하고 많은 부분을 차지하는 부모님께 감사드립니다. 자식들 뒷바라지 하느라 많은 것을 희생하신 우리 부모님. 아버지, 어머니의 존재 자체만으로도 저에게는 그 무엇보다 큰 힘이 되었습니다. 이제는 제가 아버지, 어머니의 든든한 기둥이 되어 얼굴에 항상 미소가 끊이지 않도록 노력하겠습니다. 사랑해요 ๑.

p.s. 본문에 구체적으로 적지는 않았지만, 우리 사랑하는 누님, 이윤환 중대장님, 군 선배님 및 후배, 백두산 부대와 승리부대에서의 동생들, 그리고 켄터키 대학에서 물심양면으로 많은 도움을 주신 형님, 누님들께도 감사드립니다. 


\section{TABLE OF CONTENTS}

Acknowledgements......................................................ii

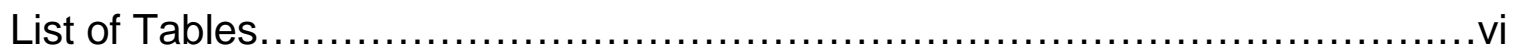

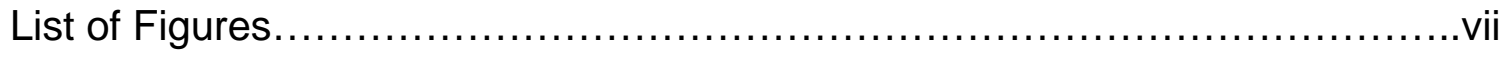

Chapter One: Background of the Drosophila neuromuscular junction and

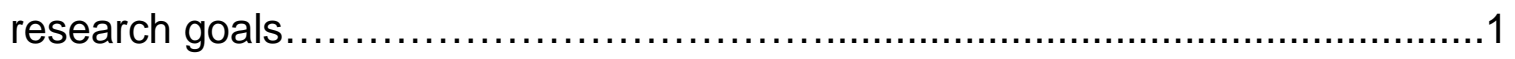

Chapter Two: Furthering pharmacological and physiological assessment of the glutamatergic receptors at the Drosophila neuromuscular junction.............50

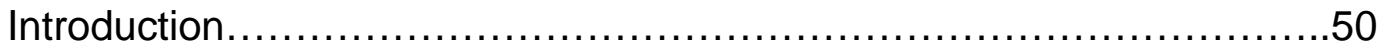

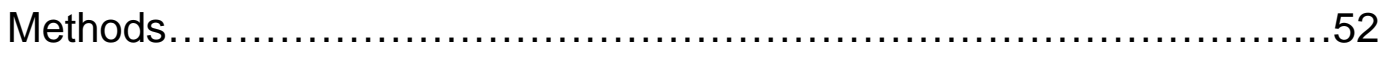

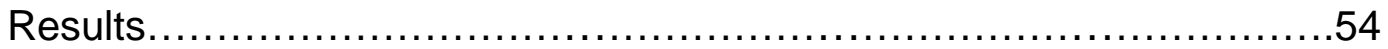

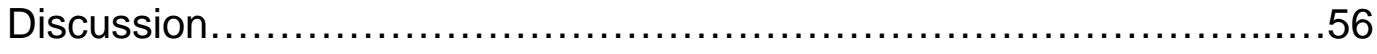

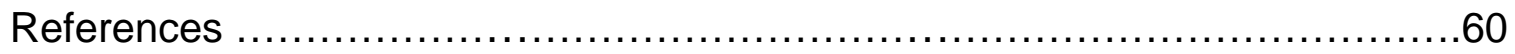

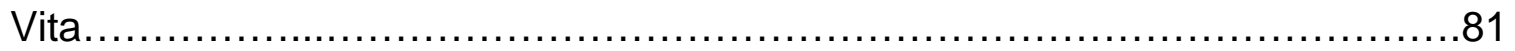




\section{LIST OF TABLES}

Table 1-1. Glutamate receptor genes in Drosophila genome...................49 


\section{LIST OF FIGURES}

Figure 1-1. Schematic model of predicted kainate action at the Drosophila NMJ.5

Figure 1-2. Schematic model of facilitation effect on presynaptic nerve

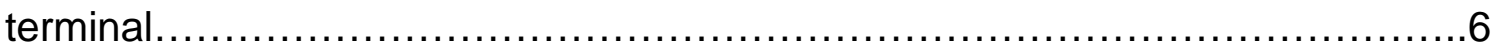

Figure 1-3. Schematic model of predicted ATPA action at Drosophila NMJ.........7

Figure 1-4. Schematic model of predicted t-ACPD action at Drosophila NMJ.......8

Figure 1-5a. Sequence alignment with predicted amino acid sequences...........9

Figure 1-5b. Sequence alignment with predicted amino acid sequences.........24

Figure 2-1. The dissected $3^{\text {rd }}$ instar Drosophila larva preparation......................70

Figure 2-2. The effect of kainate on the evoked EPSP amplitudes................71

Figure 2-3. Effects of domoic acid at the NMJ.................................72

Figure 2-4. Effects of kainate ( 1 and $0.5 \mathrm{mM}$ ) on mEPSPs properties............73

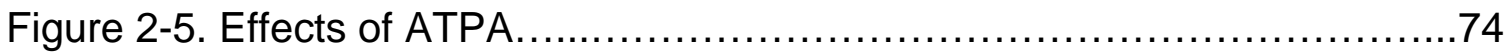

Figure 2-6. Effects of kainate on short-term depression (i.e., negative facilitation)

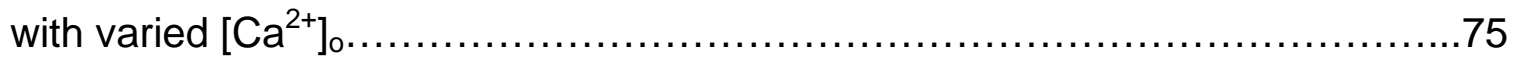

Figure 2-7. Effects of kainate (KA) and calcium on FI.........................76

Figure 2-8. The standard deviation (SD) for each preparation before and after

exposure to kainate $(1 \mathrm{mM})$ with $1 \mathrm{mM}\left[\mathrm{Ca}^{2+}\right]_{\mathrm{o}}$

Figure 2-9. Action of t-ACPD on evoked EPSPs and resting membrane

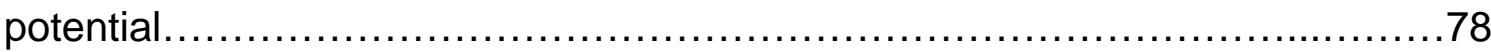

Figure 2-10. Sequence comparison of five postsynaptic receptor subunits.....79

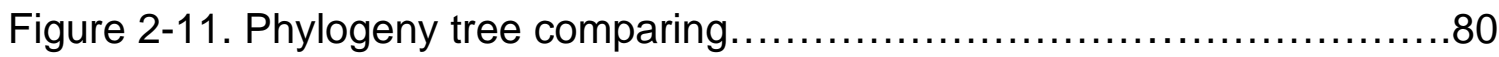




\section{Chapter One}

\section{Background of the Drosophila neuromuscular junction and research goals}

The nervous system in animals is used to convey information of the surrounding external and internal environment, as well as to react to it. In the majority of cases, the electrical signals neurons use are relayed to target cells by chemical communication. When this occurs for close targets, the site of communication is referred to as a synapse. The chemical released from the presynaptic neuron is referred to as transmitter. This transmitter enters into the synaptic cleft and then binds to receptors on a target cell. The target cell can be a neuron, a muscle or an endocrine cell or even a combination of cell types. The post-synaptic receptors determine if the target cell will be excited or inhibited. These determine actions by the receptor itself, being a ligand-gated ion channel or by initiation of a cellular cascade to influence ion channels (Berridge, 1998; Miyazaki, 1995). Receptors that mediate secondary cascades are referred to as metabotropic receptors, whereas receptors that act as a receptor mediated ion channel are termed ionotropic receptors.

The neurotransmitter molecules are stored in vesicles within the presynaptic nerve terminal. These vesicles fuse to the presynaptic membrane with evoked stimulation because of the rise in $\left[\mathrm{Ca}^{2+}\right]_{i}$ (Sudhof, 2004). The $\left[\mathrm{Ca}^{2+}\right]_{i}$ is generally very low compared to that outside the neuron $\left(\left[\mathrm{Ca}^{2+}\right]_{0}\right.$ is $\left.10 \mathrm{mM}\right)$. The entry of $\mathrm{Ca}^{2+}$ into the nerve terminal causes the mobilization of both the reserve and the readily releasable pools of vesicles (Rosenmund and Stevens, 1996; Kuromi and Kidokoro, 1998).

The whole process of vesicle fusion must be finely regulated as not to over or under excite the target cells or else the information will not be correctly conveyed. Thus, there are various levels of excitation and inhibition to regulate nerve terminals, such as ion pumps, ion exchanges and auto-receptors for negative feedback. Because of the difficulty in accessibility of postsynaptic dendrites and the wide complexity of the central nervous system in general, most of our understanding in synaptic properties at a vesicle level (i.e., quantal level) has been gained by studying accessible and relatively simple preparations, such as neuromuscular junctions (NMJ) in invertebrates and amphibians (Bennett and Pettigrew, 1975; Nudell and Grinnell, 1983; Wilkinson and Lunin, 1994; Wilkinson et al., 1992). The frog, locust and crayfish neuromuscular junctions were initially used because of the relatively ease for accessibility and viability in relatively mimimal physiological salines (Fatt and Katz, 1953a, b; Dudel, 1965, 1981; Dudel et al., 1983). 
More recently the Drosophila melanogaster has become a model for synaptic transmission at the larval NMJ. This is because a known genome that can be manipulated for altered protein expression to examine mechanistic cellular functions of synapses (Atwood et al., 1993; Betz et al. 1993; Jan and Jan 1976; Kurdyak et al., 1994; Li et al., 2002; Li and Cooper, 2001; Pawlu et al., 2004; Ruffner et al., 1999; Sigrist et al., 2002, 2003; Stewart et al., 1994, 1996). A recent review in $\mathrm{Ca}^{2+}$ regulation which influences synaptic transmission in the preparation has appeared (Desai-Shah and Cooper, 2009).

Since the larval Drosophila NMJ is becoming a synaptic model of choice recently for many reasons, it is important to understand the pharmacological and physiological profiles of this synapse. The Drosophila NMJ consists of excitatory motor neurons and muscle. Recent studies described which postsynaptic receptor subtypes are expressed in muscle. There are five different subunits, which fall into kainate/AMPA type glutamate receptors based on molecular sequence homology (Betz et al., 1993; Völkner et al., 2000; Marrus et al., 2004; Qin et al., 2005; Guerrero et al., 2005). However, in an experiment investigating a pharmacological sensitivity on the postsynaptic receptors for intact $3^{\text {rd }}$ instar Drosophila NMJ, kainate did not depolarize the muscle but reduced the EPSP amplitude. Also, AMPA did not alter the EPSP amplitude nor alter the resting membrane potential (Bhatt and Cooper, 2005). Thus, the postsynaptic receptors at the Drosophila NMJ should not be classified based on a pharmacological profiling nomenclature as a kainate/AMPA subtype of glutamate receptors. Based on my investigations and the previous study (Bhatt and Cooper, 2005), kainate appears to be blocking the glutamate activities at the larval Drosophila NMJ.

To date, the pharmacological assessment of this NMJ has not been tackled carefully. No one has even addressed if the motor nerve terminals may possess glutamate auto-receptors as noted to occur in other arthropod species (Schramm and Dudel, 1997). Thus, I choose to address these issues in my studies.

In this research project, I first support the idea that the postsynaptic receptors at the Drosophila NMJ are not of a kainate subtype of glutamate receptors and that application of kainate reduces the evoked EPSP amplitude. I approached these studies by use of pharmacological and physiological investigations. Specifically I addressed the spontaneous vesicular postsynaptic responses at the $3^{\text {rd }}$ instar NMJ using domoic acid and glutamate. Domoic acid is a potent agonist of the kainate subtype of glutamate receptors in vertebrates. I also addressed how exogenous application of kainate could be causing the reduction of the EPSP amplitudes.

The hypotheses I am testing is based on preliminary findings at this $\mathrm{NMJ}$ and combined reports on other glutamatergic synapses in various animal 
models. The hypotheses addresses why kainate produces a reduction in the EPSP amplitude. My hypotheses are as following;

1) Kainate has a direct postsynaptic action as an antagonist.

2) Kainate has a direct presynaptic action reducing glutamate release, possibly through activating glutamatergic autoreceptors on the nerve terminals.

Addressing these aims is very important in understanding processes in synaptic regulation at the neuromuscular junction of this model preparation. Several disorders are known for altered synaptic function in the vertebrate brain. In order to understand these disorders better, one can use invertebrate models such as Drosophila, which can serve as good electrophysiological and genetic models to study synaptic regulation on a fundamental level.

In order to elucidate my hypotheses about kainate's action at the Drosophila NMJ, I conducted several experiments as described in detail within Chapter Two. In short, I examined the action of kainate on spontaneous fusion of vesicles with exposure to kainate. This is diagrammatically shown in Figure 1-1 with kainate either having an action on the presynaptic terminal or having an action on the postsynaptic receptors as to alter receptivity to glutamate. Since $\left[\mathrm{Ca}^{2+}\right]_{\mathrm{i}}$ is tightly regulated within the nerve terminal for rapid transmission, if kainate had a presynaptic action via alteration in the $\left[\mathrm{Ca}^{2+}\right]_{\mathrm{i}}$, I would predict that short-term facilitation or depression, known to occur at this synaptic preparation, would be greatly affected. Thus, I used a four-pulse stimulation train and measured the facilitation index before and during application of kainate at various concentrations of kainate as well as $\left[\mathrm{Ca}^{2+}\right]_{0}$. Since transmission is so sensitive to $\left[\mathrm{Ca}^{2+}\right]_{\mathrm{o}}$ altering the concentration with various concentration of kainate exposure might unmask any subtle effects of kainate. This general model to address this experimental paradigm is shown in Figure 1-2. In addition to the action of kainate other pharmacological agents were examined to help in describing the pharmacological profiles of the putative presynaptic and known postsynaptic glutamatergic receptor subtypes. The working models of action for ATPA (Figure 1-3) and t-ACPD (Figure 1-4) are shown for action on evoked transmission.

In addition, the reports which characterized the molecular sequence of the glutamate receptors at the Drosophila NMJ to the human's kainate receptor subunits, did not provide the statistical similarities between them. The paper published in 2005 by Qin et al., designated some amino acid sequences as similar even though they appear to be far from being similar. Besides, in this earlier study only five amino acid sequences of the postsynaptic receptor subunits at the Drosophila NMJ were examined although there are around 30 different glutamate receptor genes in Drosophila genome (Littleton and Ganetzky, 
2000). Thus, I show statistical similarities of the aligned sequences (Figure 1$\mathbf{5 a , b )}$ and provide a phylogeny tree with the known 36 predicted amino acid sequences of the glutamate receptors in Drosophila genome (Table 1-1). 


\section{Model of a Neuromuscular Juncction}

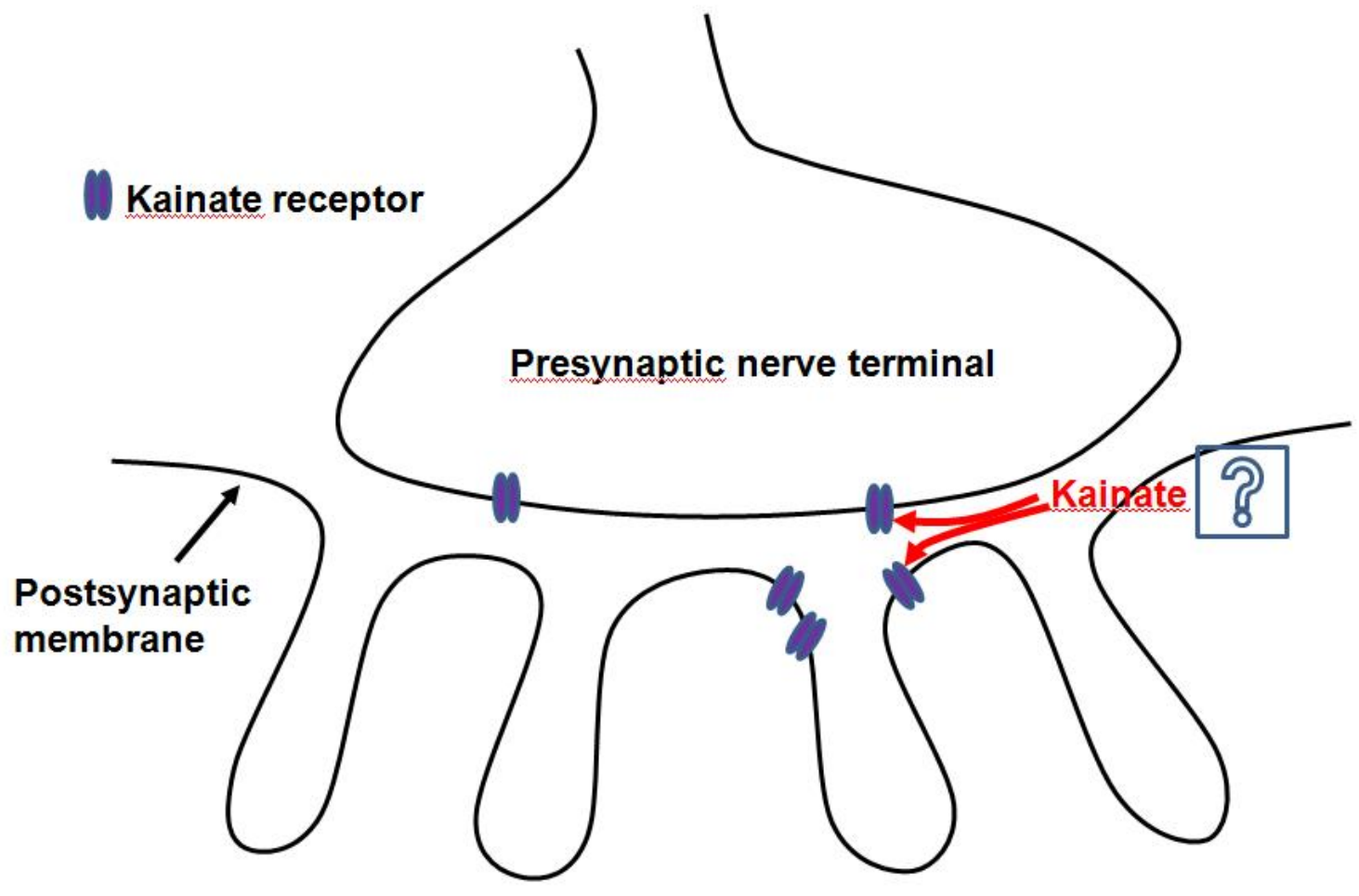

Figure 1-1. Schematic model of predicted kainate action at the Drosophila neuromuscular junction, which is the site of communication from the axon terminal of motorneuron to the muscle. Kainate is added into the synaptic cleft without neural stimulation to measure any possible direct effect on either the presynaptic nerve terminal or on postsynaptic sensitivity. Spontaneous responses are involved in one vesicle fusion events. If kainate has an effect presynaptically then one would expect an alteration in the frequency of spontaneous response. If there was an affect on the postsynaptic receptors then one would expect a change in the amplitudes or shape of the spontaneous events. The frequency and amplitude of spontaneous responses before and during exposure to kainate are compared. 


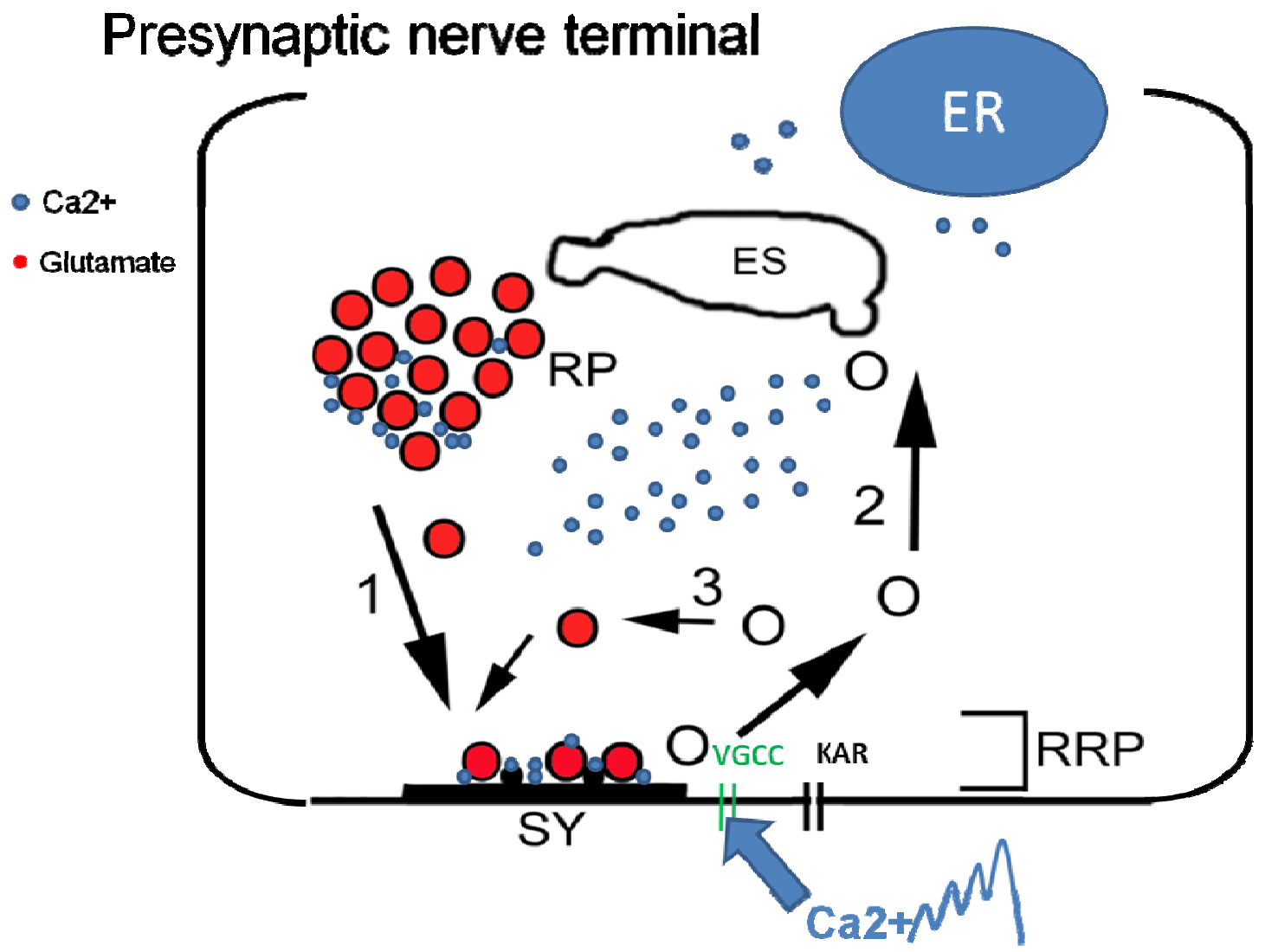

Figure 1-2. Schematic model of facilitation effect on presynaptic nerve terminal. $\mathrm{Ca}^{2+}$ plays a critical role in vesicle fusion in the presynaptic nerve terminal. $\left[\mathrm{Ca}^{2+}\right]$ in the presynaptic nerve terminal can be changed at each pulse of induced facilitation. Four-pulses with $20 \mathrm{~Hz}$ is induced in the presynaptic nerve terminal, which may alter the dynamics affecting several factors involved in vesicle fusion inside the cell. This presynaptic activity at each pulse will have a slightly different condition. If there is a kainate effect on $\mathrm{Ca}^{2+}$ dynamics it would likely alter the vesicle fusion events during facilitation. Facilitation Index $(\mathrm{FI})\left(4^{\text {th }}\right.$ pulse amplitude / $1^{\text {st }}$ pulse amplitude -1 ) is measured before and during kainate exposure to determine if there is a presynaptic action of kainate. (RP: reserve pool, RRP: readily releasable pool, ER: intracellular $\mathrm{Ca}^{2+}$ storage, ES: endosome, VGCC: voltage-gated $\mathrm{Ca}^{2+}$ channel, KAR: kainate receptor) 


\section{Model of a Neuromuscular Junction}

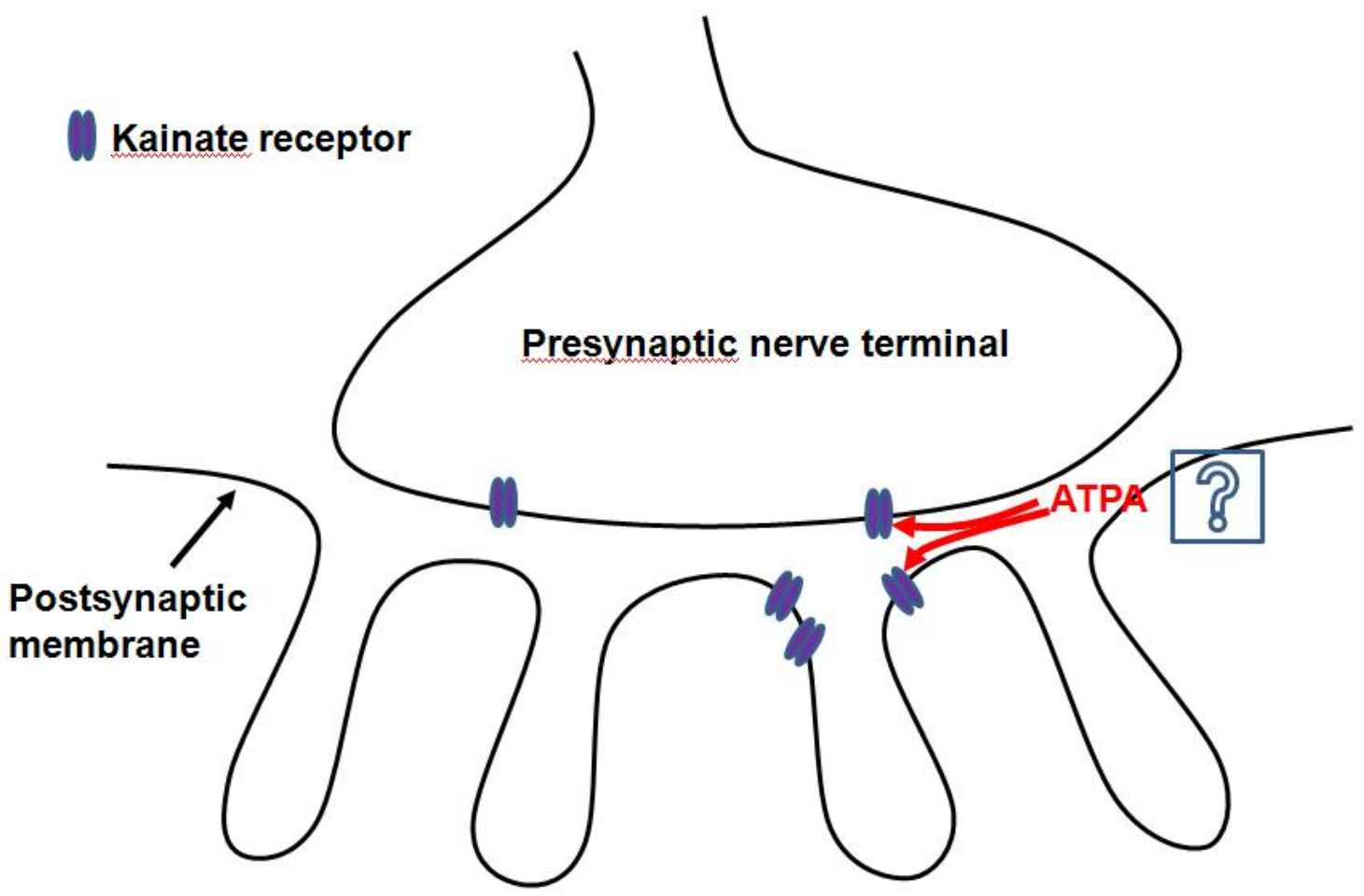

Figure 1- 3. Schematic model of predicted ATPA action at Drosophila NMJ. (RS)-2-Amino-3-(3-hydroxy-5-tert-butylisoxazol-4-yl)propanoic acid (ATPA) is a kainate receptor agonist. If ATPA has an action either presynaptically or postsynaptically it would alter the frequency of amplitude of spontaneous events and the size of evoked responses. Excitatory postsynaptic potential (EPSP) amplitudes and membrane potential in the muscle are measured with the bath application of ATPA to compare if they are affected. 


\section{Model of a \\ Neuromuscular Junction}

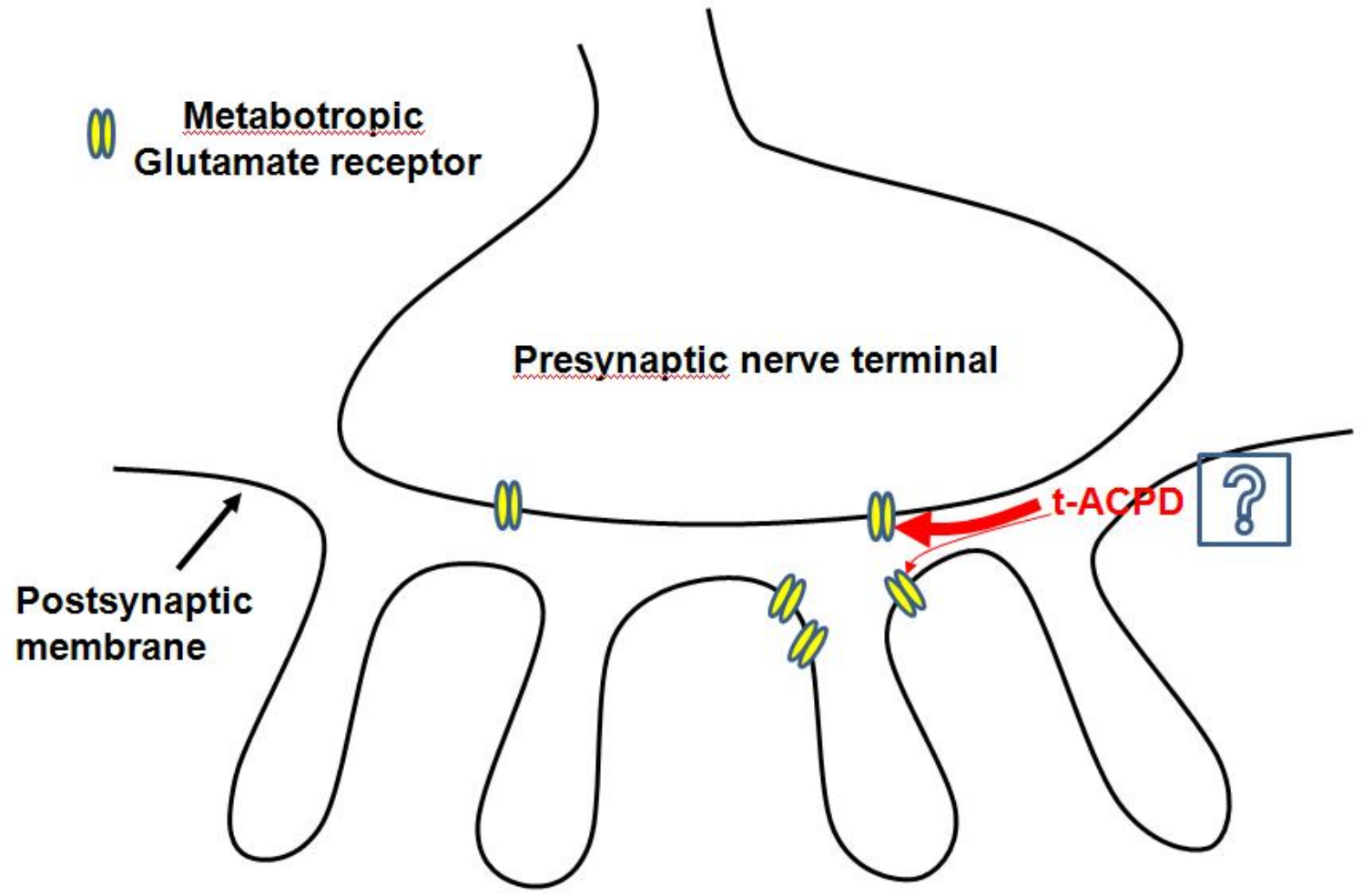

Figure 1- 4. Schematic model of predicted t-ACPD action at Drosophila NMJ. trans-( \pm )-1-Amino-1,3-cyclopentanedicarboxylic acid monohydrate (t-ACPD) is a well-known metabotropic glutamate receptor agonist. If $\mathrm{t}$-ACPD has an action either presynaptically or postsynaptically it would alter the amplitude of evoked response. Excitatory postsynaptic potential (EPSP) amplitudes and membrane potential in the evoked response are measured with the bath application of tACPD to see if there is a t-ACPD action through the metabotropic glutamate receptors. In the figure, narrow arrow is described to show the action of t-ACPD on the postsynaptic receptor because of a small possibility of a postsynaptic action on receptors at the Drosophila NMJ. The receptors are known as ionotropic glutamate receptors. 
- - MRLCPVIYAFIIIIGFLEGIIALGGDDRNEITVG - - -AIFYENEKEIELSFDQAFREVN - NMKFSELRFVTIKRYMPTNDSFLLQQ

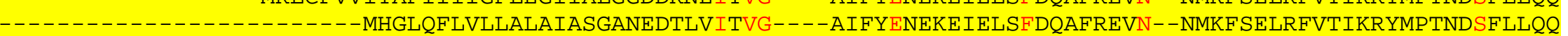
MKKELSGNSSARWPQMFGGNSREAGMWQRILLLGCMWSAFFMCRSRGQQINIG - - - - AFFYDDELELEKEFMTVVNAINGPESEQTMRFYPLIKRLKPEDGSVTMQE - - - - - MHFCWIS - - LIILSLSRVQAQFYGGNAYEASSGQSIRLG - - - LITDDATDRIRQTFEHAISVVN - - - NELGVPLVGETEQVAYGNSVQAFA - - - - - - - - MFFNHFVILWSLFSIHISVNWAQYENFGG - YDNYQSLESVPIG - - - LLTDQNTEQMNIVFDHAIDVAN - - - QEVGTSLTSLKEEVNYGDAYQSYG - . - . - . - MEHGTLLAQPGLWTRDTSWALLYFLCYILPQTAP - QVLRIGGIFETVENEPVNVEELAFKFAVTSINRNRTLMPNTTLTYDIQRINLFDSFEASR

ITCELISNGVAAIFGPSSKAASDIVAQIANATGIPHIEYDLKLEATRQEQLNHQMSINVAPSLSVLSRAYFEIIKSN - YEWRTFTLIYETPEG - - LARLQDLMNIQA ITCELISNGVAAIFGPSSKAASDIVAQIANATGIPHIEYDLKLQQSNRERMNHQLTVNVAPMELFLSAAFSDILASKTFDWKSFTIAYERSSH - - LIRLQHILAWKQ HACDLIDNGVAAIFGPSSKAASDIVALVCNSTGIPHIEFDISDEGIQAEKPNHQMTLNLYPAQAI LSKAYADIVQNF - - GWRKFTIVYDADDARAAARLQDLLQLRE QLCRLMOSGVGAVFGPAARHTASHLLNACDSKDIPFIYPHLSWGSNPDG - - - - - - FNLHPSPEDIANALYDIVNQF - - EWSRFIFCYESAEY - - LKILDHLMTRYG KLCRMLETGIAGVFGPSSRHTAVHLMSICDAMDIPHIYSYMS - - ENAEG - - - - - - - FNLHPHPADLAKALYSLITEF - - NWTRFIFLYESAEY - - LNILNELTTMLG RACDQLALGVAALFGPSHSSSVSAVQSICNALEVPHIQTRWKHPSVDNKDL - - FYINLYPDYAAISRAILDLVLYY - - NWKTVTVVYEDSTG - - LIRLQELIKAPS

LNSDYVKLRNLADYADDYRILWKETDETFHEQRIILDCEPKTLKELLKVSIDFKLQGPFRNWFLTHLDTHNSGLRDIYN - EDFKANITSVRLKVVDANPFERKKTR LHKAGIKMQEFE - RGDDYRILWKRINN - AREKFVLLDCPSDILVDVINASIGYNMTGSFNHLFLTNLDTHLSGIDGFYS - RDFTVAVAAVRIRTYVPPPVHDEIDV VHNDVVRVRKFH - KDDDFRVMWKSIRG - - ERRVVLDCEPNMLVELLNSSTEFGLTGQYNHIFLTNLETYTDHLEELAADNETFAVNITAARLLVNPDPPPYSLPYG IKGPVIKVMRYDLNLNGNYKSVLRRIRKSEDSRIVVVGSTTGVAELLRQAQQVGIMNEDYTYIIGNLNLHTFDLEEYKYS - - - EANITGIRMFSPDQEEVRDLMEK KSGTVITVLRYDMQLNGNYKQVLRRVRKSVDNRIVVVGSSETMPEFLNQAQQVGI INEDYKYIIGNLDFHSFDLEEYKYS - - - EANITGLRLFSPEKMAVKELLMK RYNIKIKIRQLPSGNK - DAKPLLKEMKKGKEFYVIFDCSHETAAEILKQILFMGMMTEYYHYFFTTLDLFALDLELYRYS - - - GVNMTGFRLLNIDNPHVSSIIEK

LT-KVDQILGN - - - - - - - QTMLPILIYDAVVLFASSARNVIAAMQP - FHPP - - - NRHCGSSSPWMLGAFIVNEMKTISEDDVEPHFKTENMKLDEYGQRIHFNL FDNSVDTRFS - - - - - - - - SLGSQLVYDSIVLFYN - ALLEISQRPG - FYIP - - - NFSCG - RGFWQPGPRLVEQMKQITPKMVKPPFKTQRLQINADGQREDFNL YVTQRDNIVYES - - - SDPPRTLIHDLIHDALQLFAQSWRNASFFYPDRMVVPRITCDFAASGGRTWAMGRYLARLMKGTSG - VNNTNFRTSILQFDEDGQRITFNI LHQELGESEP - - - VNSGSTFITMEMALTYDAVRVIAETTKHLPYQPQM - - - - - - LNCSERHDNVQPDGSTFRNYMR - - SLEIKEKTITGRIYFEGNVRKGFTFDV

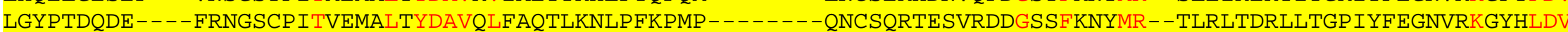
WSMERLQAPPRPETGLLDGMMTTEAALMYDAVYMVAIASHRASQLTVS - - - - - -SLQCHRHKPWR - LGPRFMNLIK - - EARWDGLTGHITFNKTNGLRKDFDLDI Figure 1-5a. Sequence alignment with predicted amino acid sequences of the postsynaptic receptor subunits at Drosophila NMJ and human's kainate receptor subunits. Compared subunits are shown on the left of the first row in aligned sequences. Identical and similar amino acids matched are colored by red and putative extracellular domains are shaded by yellow, respectively. ClustalW2 is used for sequence analysis. 
(Figure 1-5a cont.)

EIYKPTVNEPMMVWTPDN - - -GIKKRLLN - - - LELESAGTTQDFSEQRKVYTVVTHYEEPYFMMKEDHE - - NFRGREKYEGYAVDLISKLSELMEFDYEFMIVNGN EVYNPIIDRVTHIWNKEFQLVDFEKLRENSTQALKQKRLQNKEDFSQKPIRYTVATRVGKPYFSWREEPEGVHYEGNERFEGYAVDLIYMLAQECKFDFNFEPVRDN EVYDPLDGIGIAIWDPRG - - - - - - - - - QITQLNVDVKAQKKMIYRVATRIGPPYFSYNETARELNLTGNALYQGYAVDLIDAIARHVGFEYVFVPVADQ IELQTSGLVKVGTWEEGK - - - - - DFEFQR - PPQAVNFNDIDDGSLVNKTFIVLISVATKPYASLVESID - - TLIGNNQFQGYGVDLIKELADKLGFNFTFRDGGND IELQPSGIVKVGTWDEDR - - - - - QYRPQRLAPTTAQFDSVDN - SLANKTFIILLSVPNKPYAQLVETYK - - QLEGNSQYEGYGVDLIKELADKLGFNFTFVNGGND ISLKEEGTEKIGIWNSNS - - - - - - GLNMTD - - -SNKDKSSNITDSLANRTLIVTT - ILEEPYVMYRKSDK - - PLYGNDRFEGYCLDLLKELSNILGFIYDVKLVPDG

TM1

- - GKYNPETKOWDGIIRKLIDHHAQIGVCDLTITOMRRSVVDFTVPFMQLGISILHYKSPPEPKNOFAFLEPFAVEVWIYMIFAQLIMTLAFVFIARLSYREWLPPN KYGSYDANTDEWDGI IRQLIDNNAQIGICDLTITQARRSVVDFTVPFMQLGISILSYKEPPPKADIYAFLNPYNAEVWLFVMIAMMITAFALIFTGRIDQYEWDQPV QYGKLDKETKQWNGIIGEI INNDAHMGICDLTITQARKTAVDFTVPFMQLGVSILAYKSPHVEKTLDAYLAPFGGEVWIWILISVFVMTFLKTIVARISKMDWENPH -YGSFNKTTNSTSGMLKEIVEGRADLAITDLTITSEREEVIDFSIPFMNLGIAILYVKPQKAPPALFSFMDPFSSEVWLYLGIAYLGVSLCFFIIGRLSPIEWDNPY - YGSYNKSTNESTGMLREIMTGRADLAITDLTITSEREQALDFTIPFMNLGIAILYLKPQKATPELFTFMDPFSEEVWWFLGFSFLGVSLSFFILGRLSPSEWDNPY -KYGAQNDKGEWNGMVKELIDHRADLAVAPLTITYVREKVIDFSKPFMTLGISILYRKPNGTNPGVFSFLNPLSPDIWMYVLLACLGVSCVLFVIARFTPYEWYNPH

TM2

TM3

PAIQDPDELENIWNVNNSTWLMVGSIMQQGCDILPRGPHMRILTGMWWFFALMMLSTYTANLAAFLTSNKWQSSIKSLQDLIEQD -KVHFGSMRGGSTSLFFSESND ENVNREMERQNIWHLSNALWLVLGSMLNQGCDLLPRGLPMRLLTAFWWIFALLISQTYIAKLAAFITSSKIAGDIGSLHDLVDQN - KVQFGTIRGGATSVYFSESND PCNRDPEVLENQWRIHNTGWLTVASIMTAGCDILPRSPQVRMFEATWWIFAIIIANSYTANLAAFLTSSKMEGSIANLKDLSAQK -KVKFGTIYGGSTYNLLADSNE PCIEEPEELENQFTINNSLWFTTGALLQQGSEIAPKALSTRTISAIWWFFTLIMVSSYTANLAAFLTIENPTSPINSVKDLADNKDDVQYGAKRTGSTRNFFSTSEE PCIEEPEELENQFTLGNSIWFTTGALLQQGSEIGPKALSTRTVASFWWFFTLIVVSSYTANLAAFLTIEKPQSLINSVDDLADNKDGVVYGAKKTGSTRNFFMTSAE PCNPDSDVVENNFTLLNSFWFGVGALMQQGSELMPKALSTRIVGGIWWFFTLIIISSYTANLAAFLTVERMESPIDSADDLAKQT -KIEYGAVRDGSTMTFFKKSKI

TDYQRAWNQMKDFNPSAFTSTNKEGVARVRKEKGGYAFLMETTSLTYNIERNCDLTQIGEQIGEKHYGLAVPLGSDYRTNLSVSILQLSERGELQKMKNKWWKNHNV TDNRMAWNKMLSFKPDAFTKNNEEGVDRVKLSKGTYAFLMETTNLOYYVORNCELTOIGESFGEKHYGIAVPLNADFRSNLSVGILRLSERGELFKLRNKWFNSNES TVYRLAFNLMNNDDPSAYTKDNLEGVDRVRKNRGDYMFLMETTTLEYHREQNCDLRSVGEKFGEKHYAIAVPFGAEYRSNLSVAILKLSERGELYDLKQKWWKNPNA PIYIKMNEYLNA-HPEMLMENNQQGVDKVKSG - TKYAFLMESTSIEFNTVRECNLTKVGDPLDEKGYGIAMVKNWPYRDKFNKALLELQEQGVLARLKNKWWNEVGA ERYKKMNKFMSE - NPQYLTEDNMEGVNRVKTN-THYAFLMESTSIEYNTKRECNLKKIGDALDEKGYGIAMRKDWPHRGKFNNALLELQEQGVLEKMKNKWWNEVGT STYEKMWAFMSSRQQTALVRNSDEGIQRVLT - - TDYALLMESTSIEYVTQRNCNLTQIGGLIDSKGYGVGTPIGSPYRDKITIAILQLQEEGKLHMMKEKWWRGNG - 
(Figure 1-5a cont.)

\section{TM4}

- TCDS - YHEVDGDELSIIELGGVFLVLAGGVLIGVILGIFEFLWNVQNVAVEERVTPWQAFKAELIFALKFWVRKKPMRISS - - SSDKSSSRRSS - - - - - GSRRSSK - TCDSNVPTIDDGQFDMDSVGGLFVVLIVGVVVGLVIGVAEFLWHVQRISVKEKIPPMLALKAEFYFVIRFWLTRKPLHTYR - - QSRDSTSTGYSSLEQITSASSAK - SCFEEPDPDATPDMTFEELRGIFYTLYAGILIAFLIGITEFLVYVQQVALEERLTFKDAFKKEIRFVLCVWNNRKPIVAGTPISSVRTTPRRSLDKSLDRTPKSSR GVCSAKSDDDGPSELGVDNLSGIYVVLVIGSIISIIISILCWCYFVYKKAKNYEVPFCDALAEEFRIVIRFSENERPLKSAQSIYSRSRNSSQSIESLKTDSEENMP GICATKEDAPDATPLDMNNLEGVFFVLLVGSCCALLYGIISWVLFVMKKAHHYRVPLRDALKEEFQFVIDFNNYVRVLKNSASIYSRSRQSSMSVASVAQESQ - - - - - - CPEEDNKEASALGVENIGGIFIVLAAGLVLSVFVAIGEFIYKSRKNNDIEQCLSFNAIMEELGISLKNQKKIK - KKSRTKGKSSFTSILTCHQRRTQRKETVA

EKSRSKTVS - - - - - - - - - - - - - - - - -

KKKKTRRIEK- - - - - - - - - - - - - - - - -

RVVIGRSSEEMREMAQGSGSSSGSNNAGRGEKEARV

VED-- - - - - - - - - - - - - - - - - - - -

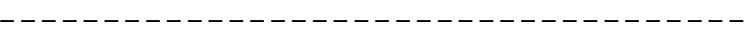

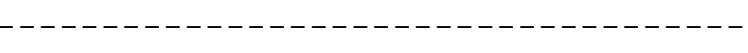


(Figure 1-5a cont.)

GluR-IIA - - - - - - ARLCPVVIYAFIIIIIGFLEGIIALGGDDRNEITVG - - - -

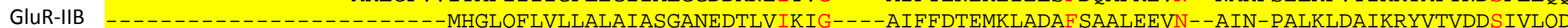
GluR-IIC MKKELSGNSSARWPQMFGGNSREAGMWQRILLLGCMWSAFFMCRSRGQQINIG - - - AFFYDDELELEKEFMTVVNAINGPESEQTMRFYPLIKRLKPEDGSVTMQE GluR-IID - - - - - - MHFCWIS - - LIILSLSRVQAQFYGGNAYEASSGQSIRLG - - - LITDDATDRIRQTFEHAISVVN - - - NELGVPLVGETEQVAYGNSVQAFA GluR-IIE - - - - - - - - - LFFNHFVILWSLFSIHISVNWAQYENFGG - YDNYQSLESVPIG - L - - - - QEVGTSLTSLKEEVNYGDAYQSYG GluR-6 - - . - . - MKIIFPILSNPVFRRTVKLLLCLLWIG - YSQGTTHVLRFGGIFEYVESGPMGAEELAFRFAVNTINRNRTLLPNTTLTYDTQKINLYDSFEASK

ITCELISNGVAAIFGPSSKAASDIVAQIANATGIPHIEYDLKLEATRQEQLNHQMSINVAPSLSVLSRAYFEIIKSN - YEWRTFTLIYETPEG - - LARLQDLMNIQA ISCDLIGSGVAAIFGPSSKTNSDIVEVLCNMTGIPHLQFDWHPQQSNRERMNHQLTVNVAPMELFLSAAFSDILASKTFDWKSFTIAYERSSH - - LIRLQHILAWKQ HACDLIDNGVAAIFGPSSKAASDIVALVCNSTGIPHIEFDISDEGIQAEKPNHQMTLNLYPAQAILSKAYADIVQNF - - GWRKFTIVYDADDARAAARLQDLLQLRE QLCRLMOSGVGAVFGPAARHTASHLLNACDSKDIPFIYP - - - - - - HLSWGSNPDGFNLHPSPEDIANALYDIVNOF - - EWSRFIFCYESAEY - - LKILDHLMTRYG KLCRMLETGIAGVFGPSSRHTAVHLMSICDAMDIPHIYS - - - - - - YMS - - ENAEGFNLHPHPADLAKALYSLITEF - - NWTRFIFLYESAEY - - LNILNELTTMLG KACDQLSLGVAAIFGPSHSSSANAVQSICNALGVPHIQTRWK - - HQVSDNKDSFYVSLYPDFSSLSRAILDLVQFF - - KWKTVTVVYDDSTG - - LIRLQELIKAPS

LNSDYVKLRNLADYADDYRILWKETDETFHEQRI ILDCEPKTLKELLKVSIDFKLOGPFRNWFLTHLDTHNSGLRDIYN - EDFKANITSVRLKVVDANPFERKKTR LHKAGIKMQEFE - RGDDYRILWKRINN - AREKFVLLDCPSDILVDVINASIGYNMTGSFNHLFLTNLDTHLSGIDGFYS - RDFTVAVAAVRIRTYVPPPVHDEIDV VHNDVVRVRKFH - KDDDFRVMWKSIRG - - ERRVVLDCEPNMLVELLNSSTEFGLTGQYNHIFLTNLETYTDHLEELAADNETFAVNITAARLLVNPDPPPYSLPYG IKGPVIKVMRYDLNLNGNYKSVLRRIRKSEDSRIVVVGSTTGVAELLRQAQQVGIMNEDYTYIIGNLNLHTFDLEEYKYS - - - EANITGIRMFSPDQEEVRDLMEK KSGTVITVLRYDMQLNGNYKQVLRRVRKSVDNRIVVVGSSETMPEFLNQAQQVGI INEDYKYIIGNLDFHSFDLEEYKYS - - - EANITGLRLFSPEKMAVKELLMK RYNLRLKIRQLPADTK-DAKPLLKEMKRGKEFHVIFDCSHEMAAGILKQALAMGMMTEYYHYIFTTLDLFALDVEPYRYS - - - GVNMTGFRILNTENTQVSSI IEK

LT-KVDQILGN - - - - QTMLPILIYDAVVLFASSARNVIAAMQP - FHPP - - - NRHCGSSSPWMLGAFIVNEMKTISEDDVEPHFKTENMKLDEYGQRIHFNLEIYK FDNSVDTRFS - - - - - - SLGSQLVYDSIVLFYN - ALLEISQRPG - FYIP - - - NFSCG - RGFWQPGPRLVEQMKQITPKMVKPPFKTQRLQINADGQREDFNLEVYN YVTQRDNIVYESSDPPRTLIHDLIHDALQLFAQSWRNASFFYPDRMVVPRITCDFAASGGRTWAMGRYLARLMKGTSG - VNNTNFRTSILQFDEDGQRITFNIEVYD LHQELGESEP - - - - - - VNSGSTFITMEMALTYDAVRVIAETTKHLPYQP - - - - QMLNCSERHDNVQPDGSTFRNYMRSLEIKEKTITGRIYFEGNVRKGFTFDVIE LGYPTDQDE - - - - - - - FRNGSCPITVEMALTYDAVQLFAOTLKNLPFKP - - - - - MPONCSORTESVRDDGSSFKNYMRTLRLTDRLLTGPIYFEGNVRKGYHLDVIE WSMERLQAPPK - - -PDSGLLDGFMTTDAALMYDAVHVVSVAVQQFPQMT - - - - VSSLQCNRHKPWR - FGTRFMSLIKEAHWEGLTGRITFNKTNGLRTDFDLDVIS

PTVNEPMMVWTPDN - - GIKKRLLN - - - LELESAGTTQDFSEQRKVYTVVTHYEEPYFMMKEDHE - - NFRGREKYEGYAVDLISKLSELMEFDYEFMIVNGN - - GK PIIDRVTHIWNKEFQLVDFEKLRENSTQALKQKRLQNKEDFSOKPIRYTVATRVGKPYFSWREEPEGVHYEGNERFEGYAVDLIYMLAQECKFDFNFEPVRDNKYGS PLDGIGIAIWDPRG - - - - - - - - - - - QITQLNVDVKAQKKMIYRVATRIGPPYFSYNETARELNLTGNALYQGYAVDLIDAIARHVGFEYVFVPVADQQYGK LQTSGLVKVGTWEE - - - - GKDFEFQR - PPQAVNFNDIDDGSLVNKTFIVLISVATKPYASLVESID - - TLIGNNQFQGYGVDLIKELADKLGFNFTFRDGGN - DYGS LQPSGIVKVGTWDE - - - - DRQYRPQRLAPTTAQFDSVDN - SLANKTFIILLSVPNKPYAQLVETYK - - QLEGNSQYEGYGVDLIKELADKLGFNFTFVNGGN - DYGS LKEEGLEKIGTWDP - - - ASGLNMTE - - - SQKGKPANITDSLSNRSLIVTTILEEPYVLFKKSDK - - PLYGNDRFEGYCIDLLRELSTILGFTYEIRLVEDGKYGA 
(Figure 1-5a cont.)

TM1

YNPETKQWDGIIRKLIDHHAQIGVCDLTITQMRRSVVDFTVPFMQLGISILHYKSPPEPKNQFAFLEPFAVEVWIYMIFAQLIMTLAFVFIARLSYREWLPPNPAIQ YDANTDEWDGIIRQLIDNNAQIGICDLTITQARRSVVDFTVPFMQLGISILSYKEPPPKADIYAFLNPYNAEVWLFVMIAMMITAFALIFTGRIDQYEWDQPVENVN LDKETKQWNGIIGEI INNDAHMGICDLTITQARKTAVDFTVPFMQLGVSILAYKSPHVEKTLDAYLAPFGGEVWIWILISVFVMTFLKTIVARISKMDWENPHPCNR FNKTTNSTSGMLKEIVEGRADLAITDLTITSEREEVIDFSIPFMNLGIAILYVKPQKAPPALFSFMDPFSSEVWLYLGIAYLGVSLCFFIIGRLSPIEWDNPYPCIE YNKSTNESTGMLREIMTGRADLAITDLTITSEREQALDFTIPFMNLGIAILYLKPQKATPELFTFMDPFSEEVWWFLGFSFLGVSLSFFILGRLSPSEWDNPYPCIE QDDANGQWNGMVRELIDHKADLAVAPLAITYVREKVIDFSKPFMTLGISILYRKPNGTNPGVFSFLNPLSPDIWMYILLAYLGVSCVLFVIARFSPYEWYNPHPCNP

TM2

TM3

DPDELENIWNVNNSTWLMVGSIMQQGCDILPRGPHMRI TTGMWWFFALMMLSTYTANLAAFLTSNKWQSSIKSLQDLIEQD - KVHFGSMRGGSTSLFFSESNDTDYQ REMERQNIWHLSNALWLVLGSMLNQGCDLLPRGLPMRLLTAFWWIFALLISQTYIAKLAAFITSSKIAGDIGSLHDLVDQN - KVQFGTIRGGATSVYFSESNDTDNR DPEVLENQWRIHNTGWLTVASIMTAGCDILPRSPQVRMFEATWWIFAIIIANSYTANLAAFLTSSKMEGSIANLKDLSAQK-KVKFGTIYGGSTYNLLADSNETVYR EPEELENQFTINNSLWFTTGALLQQGSEIAPKALSTRTISAIWWFFTLIMVSSYTANLAAFLTIENPTSPINSVKDLADNKDDVQYGAKRTGSTRNFFSTSEEPIYI EPEELENQFTLGNSIWFTTGALLQQGSEIGPKALSTRTVASFWWFFTLIVVSSYTANLAAFLTIEKPQSLINSVDDLADNKDGVVYGAKKTGSTRNFFMTSAEERYK DSDVVENNFTLLNSFWFGVGALMQQGSELMPKALSTRIVGGIWWFFTLIIISSYTANLAAFLTVERMESPIDSADDLAKQT - KIEYGAVEDGATMTFFKKSKISTYD

RAWNQMKDFNPSAFTSTNKEGVARVRKEKGGYAFLMETTSLTYNIERNCDLTQIGEQIGEKHYGLAVPLGSDYRTNLSVSILQLSERGELQKMKNKWWKNHNV-TCD MAWNKMLSFKPDAFTKNNEEGVDRVKLSKGTYAFLMETTNLQYYVQRNCELTQIGESFGEKHYGIAVPLNADFRSNLSVGILRLSERGELFKLRNKWFNSNES - TCD LAFNLMNNDDPSAYTKDNLEGVDRVRKNRGDYMFLMETTTLEYHREQNCDLRSVGEKFGEKHYAIAVPFGAEYRSNLSVAILKLSERGELYDLKOKWWKNPNA-SCF KMNEYLNAHPE - MLMENNQQGVDKVKSG - TKYAFLMESTSIEFNTVRECNLTKVGDPLDEKGYGIAMVKNWPYRDKFNKALLELQEQGVLARLKNKWWNEVGAGVCS KMNKFMSENPQ - YLTEDNMEGVNRVKTN - THYAFLMESTSIEYNTKRECNLKKIGDALDEKGYGIAMRKDWPHRGKFNNALLELQEQGVLEKMKNKWWNEVGTGICA KMWAFMSSRRQSVLVKSNEEGIQRVLT - -SDYAFLMESTTIEFVTQRNCNLTQIGGLIDSKGYGVGTPMGSPYRDKITIAILQLQEEGKLHMMKEKWWRGNG - - - C

TM4

S - YHEVDGDELSIIELGGVFLVLAGGVLIGVILGIFEFLWNVQNVAVEERVTPWQAFKAELIFALKFWVRKKPMRISS - - SSDKSSSRRSS - - - - GSRRSSKEKSR SNVPTIDDGQFDMDSVGGLFVVLIVGVVVGLVIGVAEFLWHVQRISVKEKIPPMLALKAEFYFVIRFWLTRKPLHTYR - -QSRDSTSTGYSSLEQITSASSAKKKKK EEPDPDATPDMTFEELRGIFYTLYAGILIAFLIGITEFLVYVQQVALEERLTFKDAFKKEIRFVLCVWNNRKPIVAGTPISSVRTTPRRSLDKSLDRTPKSSRRVVI AKSDDDGPSELGVDNLSGIYVVLVIGSIISI IISILCWCYFVYKKAKNYEVPFCDALAEEFRIVIRFSENERPLKSAQSIYSRSRNSSQSIESLKTDSEENMPVED TKEDAPDATPLDMNNLEGVFFVLLVGSCCALLYGIISWVLFVMKKAHHYRVPLRDALKEEFQFVIDFNNYVRVLKNSASIYSRSRQSSMSVASVAQESQ - - - - - - -

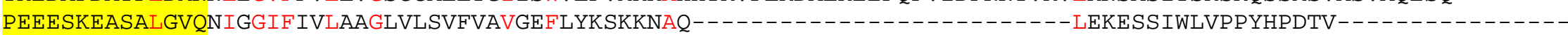


(Figure 1-5a cont.)

SKTVS- - - - - - - - - - - - - - - - - - -

TRRIEK - .

GRSSEEMREMAQGSGSSSGSNNAGRGEKEARV

-

-

- 
(Figure 1-5a cont.)

GluR-IIA - - - - - - ARLCPVVIYAFIIIIIGFLEGIIALGGDDRNEITVG - - - -

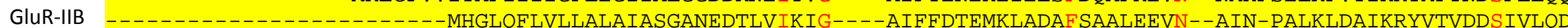
GluR-IIC MKKELSGNSSARWPQMFGGNSREAGMWQRILLLGCMWSAFFMCRSRGQQINIG - - - AFFYDDELELEKEFMTVVNAINGPESEQTMRFYPLIKRLKPEDGSVTMQE GluR-IID - - - - - - MHFCWIS - - LIILSLSRVQAQFYGGNAYEASSGQSIRLG - - - LITDDATDRIRQTFEHAISVVN - - - NELGVPLVGETEQVAYGNSVQAFA GluR-IIE - - - - - - - - - LFFNHFVILWSLFSIHISVNWAQYENFGG - YDNYQSLESVPIG - L - - - - QEVGTSLTSLKEEVNYGDAYQSYG GluR-6 - - . - . - MKIIFPILSNPVFRRTVKLLLCLLWIG - YSQGTTHVLRFGGIFEYVESGPMGAEELAFRFAVNTINRNRTLLPNTTLTYDTQKINLYDSFEASK

ITCELISNGVAAIFGPSSKAASDIVAQIANATGIPHIEYDLKLEATRQEQLNHQMSINVAPSLSVLSRAYFEIIKSN - YEWRTFTLIYETPEG - - LARLQDLMNIQA ISCDLIGSGVAAIFGPSSKTNSDIVEVLCNMTGIPHLQFDWHPQQSNRERMNHQLTVNVAPMELFLSAAFSDILASKTFDWKSFTIAYERSSH - - LIRLQHILAWKQ HACDLIDNGVAAIFGPSSKAASDIVALVCNSTGIPHIEFDISDEGIQAEKPNHQMTLNLYPAQAILSKAYADIVQNF - - GWRKFTIVYDADDARAAARLQDLLQLRE QLCRLMOSGVGAVFGPAARHTASHLLNACDSKDIPFIYP - - - - - - HLSWGSNPDGFNLHPSPEDIANALYDIVNOF - - EWSRFIFCYESAEY - - LKILDHLMTRYG KLCRMLETGIAGVFGPSSRHTAVHLMSICDAMDIPHIYS - - - - - - YMS - - ENAEGFNLHPHPADLAKALYSLITEF - - NWTRFIFLYESAEY - - LNILNELTTMLG KACDQLSLGVAAIFGPSHSSSANAVQSICNALGVPHIQTRWK - - HQVSDNKDSFYVSLYPDFSSLSRAILDLVQFF - - KWKTVTVVYDDSTG - - LIRLQELIKAPS

LNSDYVKLRNLADYADDYRILWKETDETFHEQRI ILDCEPKTLKELLKVSIDFKLOGPFRNWFLTHLDTHNSGLRDIYN - EDFKANITSVRLKVVDANPFERKKTR LHKAGIKMQEFE - RGDDYRILWKRINN - AREKFVLLDCPSDILVDVINASIGYNMTGSFNHLFLTNLDTHLSGIDGFYS - RDFTVAVAAVRIRTYVPPPVHDEIDV VHNDVVRVRKFH - KDDDFRVMWKSIRG - - ERRVVLDCEPNMLVELLNSSTEFGLTGQYNHIFLTNLETYTDHLEELAADNETFAVNITAARLLVNPDPPPYSLPYG IKGPVIKVMRYDLNLNGNYKSVLRRIRKSEDSRIVVVGSTTGVAELLRQAQQVGIMNEDYTYI IGNLNLHTFDLEEYKYS - - - EANITGIRMFSPDQEEVRDLMEK KSGTVITVLRYDMQLNGNYKQVLRRVRKSVDNRIVVVGSSETMPEFLNQAQQVGI INEDYKYIIGNLDFHSFDLEEYKYS - - - EANITGLRLFSPEKMAVKELLMK RYNLRLKIRQLPADTK-DAKPLLKEMKRGKEFHVIFDCSHEMAAGILKQALAMGMMTEYYHYIFTTLDLFALDVEPYRYS - - - GVNMTGFRILNTENTQVSSI IEK

LT-KVDQILGN - - - - QTMLPILIYDAVVLFASSARNVIAAMQP - FHPP - - - NRHCGSSSPWMLGAFIVNEMKTISEDDVEPHFKTENMKLDEYGQRIHFNLEIYK FDNSVDTRFS - - - - - - SLGSQLVYDSIVLFYN - ALLEISQRPG - FYIP - - - NFSCG - RGFWQPGPRLVEQMKQITPKMVKPPFKTQRLQINADGQREDFNLEVYN YVTQRDNIVYESSDPPRTLIHDLIHDALQLFAQSWRNASFFYPDRMVVPRITCDFAASGGRTWAMGRYLARLMKGTSG - VNNTNFRTSILQFDEDGQRITFNIEVYD LHQELGESEP - - - - - - VNSGSTFITMEMALTYDAVRVIAETTKHLPYQP - - - - QMLNCSERHDNVQPDGSTFRNYMRSLEIKEKTITGRIYFEGNVRKGFTFDVIE LGYPTDQDE - - - - - - - FRNGSCPITVEMALTYDAVQLFAOTLKNLPFKP - - - - - MPONCSORTESVRDDGSSFKNYMRTLRLTDRLLTGPIYFEGNVRKGYHLDVIE WSMERLQAPPK - - -PDSGLLDGFMTTDAALMYDAVHVVSVAVQQFPQMT - - - - VSSLQCNRHKPWR - FGTRFMSLIKEAHWEGLTGRITFNKTNGLRTDFDLDVIS

PTVNEPMMVWTPDN - - GIKKRLLN - - - LELESAGTTQDFSEQRKVYTVVTHYEEPYFMMKEDHE - - NFRGREKYEGYAVDLISKLSELMEFDYEFMIVNGN - - GK PIIDRVTHIWNKEFQLVDFEKLRENSTQALKQKRLQNKEDFSOKPIRYTVATRVGKPYFSWREEPEGVHYEGNERFEGYAVDLIYMLAQECKFDFNFEPVRDNKYGS PLDGIGIAIWDPRG - - - - - - - - - - - QITQLNVDVKAQKKMIYRVATRIGPPYFSYNETARELNLTGNALYQGYAVDLIDAIARHVGFEYVFVPVADQQYGK LQTSGLVKVGTWEE - - - - GKDFEFQR - PPQAVNFNDIDDGSLVNKTFIVLISVATKPYASLVESID - - TLIGNNQFQGYGVDLIKELADKLGFNFTFRDGGN - DYGS LQPSGIVKVGTWDE - - - - DRQYRPQRLAPTTAQFDSVDN - SLANKTFIILLSVPNKPYAQLVETYK - - QLEGNSQYEGYGVDLIKELADKLGFNFTFVNGGN - DYGS LKEEGLEKIGTWDP - - - ASGLNMTE - - - SQKGKPANITDSLSNRSLIVTTILEEPYVLFKKSDK - - PLYGNDRFEGYCIDLLRELSTILGFTYEIRLVEDGKYGA 
YNPETKQWDGIIRKLIDHHAQIGVCDLTITQMRRSVVDFTVPFMQLGISILHYKSPPEPKNQFAFLEPFAVEVWIYMIFAQLIMTLAFVFIARLSYREWLPPNPAIQ YDANTDEWDGIIRQLIDNNAQIGICDLTITQARRSVVDFTVPFMQLGISILSYKEPPPKADIYAFLNPYNAEVWLFVMIAMMITAFALIFTGRIDQYEWDQPVENVN LDKETKQWNGIIGEI INNDAHMGICDLTITQARKTAVDFTVPFMQLGVSILAYKSPHVEKTLDAYLAPFGGEVWIWILISVFVMTFLKTIVARISKMDWENPHPCNR FNKTTNSTSGMLKEIVEGRADLAITDLTITSEREEVIDFSIPFMNLGIAILYVKPQKAPPALFSFMDPFSSEVWLYLGIAYLGVSLCFFIIGRLSPIEWDNPYPCIE YNKSTNESTGMLREIMTGRADLAITDLTITSEREQALDFTIPFMNLGIAILYLKPQKATPELFTFMDPFSEEVWWFLGFSFLGVSLSFFILGRLSPSEWDNPYPCIE QDDANGQWNGMVRELIDHKADLAVAPLAITYVREKVIDFSKPFMTLGISILYRKPNGTNPGVFSFLNPLSPDIWMYILLAYLGVSCVLFVIARFSPYEWYNPHPCNP

TM2

TM3

DPDELENIWNVNNSTWLMVGSIMQQGCDILPRGPHMRILTGMWWFFALMMLSTYTANLAAFLTSNKWQSSIKSLQDLIEQD-KVHFGSMRGGSTSLFFSESNDTDYQ REMERQNIWHLSNALWLVLGSMLNQGCDLLPRGLPMRLLTAFWWIFALLISOTYIAKLAAFITSSKIAGDIGSLHDLVDON - KVQFGTIRGGATSVYFSESNDTDNR DPEVLENQWRIHNTGWLTVASIMTAGCDILPRSPQVRMFEATWWIFAIIIANSYTANLAAFLTSSKMEGSIANLKDLSAQK-KVKFGTIYGGSTYNLLADSNETVYR EPEELENQFTINNSLWFTTGALLQQGSEIAPKALSTRTISAIWWFFTLIMVSSYTANLAAFLTIENPTSPINSVKDLADNKDDVQYGAKRTGSTRNFFSTSEEPIYI EPEELENOFTLGNSIWFTTGALLOQGSEIGPKALSTRTVASFWWFFTLIVVSSYTANLAAFLTIEKPOSLINSVDDLADNKDGVVYGAKKTGSTRNFFMTSAEERYK DSDVVENNFTLLNSFWFGVGALMQQGSELMPKALSTRIVGGIWWFFTLIIISSYTANLAAFLTVERMESPIDSADDLAKQT -KIEYGAVEDGATMTFFKKSKISTYD

RAWNQMKDFNPSAFTSTNKEGVARVRKEKGGYAFLMETTSLTYNIERNCDLTQIGEQIGEKHYGLAVPLGSDYRTNLSVSILQLSERGELQKMKNKWWKNHNV-TCD MAWNKMLSFKPDAFTKNNEEGVDRVKLSKGTYAFLMETTNLQYYVQRNCELTQIGESFGEKHYGIAVPLNADFRSNLSVGILRLSERGELFKLRNKWFNSNES - TCD LAFNLMNNDDPSAYTKDNLEGVDRVRKNRGDYMFLMETTTLEYHREQNCDLRSVGEKFGEKHYAIAVPFGAEYRSNLSVAILKLSERGELYDLKQKWWKNPNA-SCF KMNEYLNAHPE - MLMENNQQGVDKVKSG - TKYAFLMESTSIEFNTVRECNLTKVGDPLDEKGYGIAMVKNWPYRDKFNKALLELQEQGVLARLKNKWWNEVGAGVCS KMNKFMSENPQ - YLTEDNMEGVNRVKTN - THYAFLMESTSIEYNTKRECNLKKIGDALDEKGYGIAMRKDWPHRGKFNNALLELQEQGVLEKMKNKWWNEVGTGICA KMWAFMSSRRQSVLVKSNEEGIQRVLT - -SDYAFLMESTTIEFVTQRNCNLTQIGGLIDSKGYGVGTPMGSPYRDKITIAILQLQEEGKLHMMKEKWWRGNG - - - C

\section{TM4}

S-YHEVDGDELSIIELGGVFLVLAGGVLIGVILGIFEFLWNVQNVAVEERVTPWQAFKAELIFALKFWVRKKPMRISS - - SSDKSSSRRSS - - - - GSRRSSKEKSR SNVPTIDDGQFDMDSVGGLFVVLIVGVVVGLVIGVAEFLWHVQRISVKEKIPPMLALKAEFYFVIRFWLTRKPLHTYR - -QSRDSTSTGYSSLEQITSASSAKKKKK EEPDPDATPDMTFEELRGIFYTLYAGILIAFLIGITEFLVYVQQVALEERLTFKDAFKKEIRFVLCVWNNRKPIVAGTPISSVRTTPRRSLDKSLDRTPKSSRRVVI AKSDDDGPSELGVDNLSGIYVVLVIGSIISIIISILCWCYFVYKKAKNYEVPFCDALAEEFRIVIRFSENERPLKSAQSIYSRSRNSSQSIESLKTDSEENMPVED TKEDAPDATPLDMNNLEGVFFVLLVGSCCALLYGIISWVLFVMKKAHHYRVPLRDALKEEFQFVIDFNNYVRVLKNSASIYSRSRQSSMSVASVAQESQ - - . - . - - -

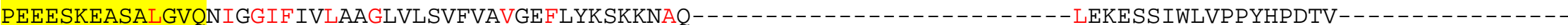


(Figure 1-5a cont.)

SKTVS- - - - - - - - - - - - - - - - - - -

TRRIEK

- -

- - - - - - - - - - - - - - -

- - - - 
(Figure 1-5a cont.)

GluR-IIA

GluR-IIB

GluR-IIC

GluR-IID

GluR-IIE

KA1

- - - - - - - - - - MRLCPVVIYAFIIIIGFLEGIIALGGDDRNEITVGAIFYE - - NEKEIELSFDQAFREVN - - NMKFSELRFVTIKRYMPTNDSFLLQQI

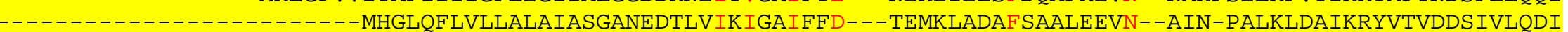
MKKELSGNSSARWPQMFGGNSREAGMWQRILLLGCMWSAFFMCRSRGQQINIGAFFYD - - - DELELEKEFMTVVNAINGPESEQTMRFYPLIKRLKPEDGSVTMQEH - - - - - - - MHFCWIS - - LIILSLSRVQAQFYGGNAYEASSGQSIRLGLITDD - - - ATDRIRQTFEHAISVVN - - - NELGVPLVGETEQVAYGNSVQAFAQ - - - - - - MFFNHFVILWSLFSIHISVNWAQYENFGG - YDNYQSLESVPIGLLTDQ - - - NTEQMNIVFDHAIDVAN - - - QEVGTSLTSLKEEVNYGDAYQSYGK - - - -

TCELISNGVAAIFGPSSK-AASDIVAQIANATGIPHIEYDLKLEATRQEQLNHQMSINVAPSLSVLSRAYFEIIKSN - YEWRTFTLIYETPEG - - LARLQDLMNIQA SCDLIGSGVAAIFGPSSK-TNSDIVEVLCNMTGIPHLQFDWHPQQSNRERMNHQLTVNVAPMELFLSAAFSDILASKTFDWKSFTIAYERSSH - - LIRLQHILAWKQ ACDLIDNGVAAIFGPSSK-AASDIVALVCNSTGIPHIEFDISDEGIQAEKPNHQMTLNLYPAQAILSKAYADIVQNF - - GWRKFTIVYDADDARAAARLQDLLQLRE LCRLMQSGVGAVFGPAAR - HTASHLLNACDSKDIPFIYPHLSWGSNPDG - - - - - FNLHPSPEDIANALYDIVNQF - - EWSRFIFCYESAEY - - LKILDHLMTRYG LCRMLETGIAGVFGPSSR - HTAVHLMSICDAMDIPHIYSYMS - - ENAEG - - - - - - FNLHPHPADLAKALYSLITEF - - NWTRFIFLYESAEY - - LNILNELTTMLG MCQILPKGVVAVLGPSSSPASSSI ISNICGEKEVPHFKVAPEEFVKFQFQR - - FTTLNLHPSNTDISVAVAGILNFF - - NCTTACLICAKAEC - - LLNLEKLLRQFL

LNSDYVKLRNLAD - YADDYRILWKETDETFHEQRIILDCEPKTLKELLKVSIDFKLQGPFRNWFLTHLDTHNSGLRDIYN - - EDFKANITSVRLKVVDANPFERKKT LHKAGIKMQEFE - - RGDDYRILWKRINN - AREKFVLLDCPSDILVDVINASIGYNMTGSFNHLFLTNLDTHLSGIDGFYS - - RDFTVAVAAVRIRTYVPPPVHDEID VHNDVVRVRKFH - -KDDDFRVMWKSIRG - - ERRVVLDCEPNMLVELLNSSTEFGLTGQYNHIFLTNLETYTDHLEELAADNETFAVNITAARLLVNPDPPPYSLPY IKGPVIKVMRYDLNLNGNYKSVLRRIRK-SEDSRIVVVGSTTGVAELLRQAQQVGIMNEDYTYI IGNLNLHTFDLEEYKYS - - - EANITGIRMFSPDQEEVRDLME KSGTVITVLRYDMQLNGNYKQVLRRVRK-SVDNRIVVVGSSETMPEFLNQAQQVGI INEDYKYI IGNLDFHSFDLEEYKYS - - - EANITGLRLFSPEKMAVKELLM ISKDTLSVRMLDD - - TRDPTPLLKEIRD - DKTAT I I IHANASMSHT ILLKAAELGMVSAYYTYIFTNLEFSLQRMDSLVDD - - - - RVNILGFSIFNQSHAFFQEFAQ

RLT - KVDQILGN - - - - - QTMLPILIYDAVVLFASSARNVIAAMQP - FHPP - - - NRHCGSSSPWMLGAFIVNEMKTISEDDVEPHFKTENMKLDEYGQRIHFNLEI VFDNSVDTRFS - - - - - - SLGSQLVYDSIVLFYN - ALLEISQRPG - FYIP - - - NFSCG - RGFWQPGPRLVEQMKQITPKMVKPPFKTQRLQINADGQREDFNLEV GYVTORDNIVYESSD - PPRTL IHDL IHDALQLFAOSWRNASFFYPDRMVVPRITCDFAASGGRTWAMGRYLARLMKGTSG - VNNTNFRTSILQFDEDGORITFNIEV KLHQELGESEPVNSGSTFITMEMALTYDAVRVIAETTKHLPYQPQM - - - - - - - LNCSERHDNVQPDGSTFRNYMR - - SLEIKEKTITGRIYFEGNVRKGFTFDVIE KLGYPTDQDE - FRNGSCPITVEMALTYDAVQLFAQTLKNLPFKPMP - - - - - - - QNCSQRTESVRDDGSSFKNYMR - - TLRLTDRLLTGPIYFEGNVRKGYHLDVIE SLNQSWQENCDHVPFTG - PALSSALLFDAVYAVVTAVQELNRSQEIG - - - - - VKPLSCGSAQIWQHGTSLMNYLR - - - MVELEGLTGHIEFNSKGQRSNYALKILQ

YKPTVNEPMMVWTPDN - - - GIKKRLLN - - - LELESAGTTQDFSEQRKVYTVVTHYE - EPYFMMKEDHE - - NFRGREKYEGYAVDLISKLSELMEFDYEFMIVNGN YNPIIDRVTHIWNKEFQLVDFEKLRENSTQALKQKRLQNKEDFSQKPIRYTVATRVG - KPYFSWREEPEGVHYEGNERFEGYAVDLIYMLAQECKFDFNFEPVRDNK YDPLDGIGIAIWDPRG - - - - - - - - - - - QITQLNVDVKAQKKMIYRVATRIG - PPYFSYNETARELNLTGNALYQGYAVDLIDAIARHVGFEYVFVPVADQQ LQTSGLVKVGTWEEGK- - - - - DFEFQR-PPQAVNFNDIDDGSLVNKTFIVLISVATKPYASLVESID - - TLIGNNQFQGYGVDLIKELADKLGFNFTFR-DGGND LQPSGIVKVGTWDEDR - - - - - QYRPQRLAPTTAQFDSVDN - SLANKTFI ILLSVPNKPYAQLVETYK - -QLEGNSQYEGYGVDLIKELADKLGFNFTFV-NGGND FTRNGFRQIGQWHVAEG - - - - - - - - - - LSMDSHLYASNISDTLFNTTLVVTTILENPYLMLKGNHQ - - EMEGNDRYEGFCVDMLKELAEILRFNYKIRLVGDGV 
(Figure 1-5a cont.)

TM1

- GKYNPETKQWDGIIRKLIDHHAQIGVCDLTITQMRRSVVDFTVPFMQLGISILHYKSPPEPKNQFAFLEPFAVEVWIYMIFAQLIMTLAFVFIARLSYREWLPPNP YGSYDANTDEWDGIIRQLIDNNAQIGICDLTITQARRSVVDFTVPFMQLGISILSYKEPPPKADIYAFLNPYNAEVWLFVMIAMMITAFALIFTGRIDQYEWDQPVE YGKLDKETKQWNGI IGEIINNDAHMGICDLTITQARKTAVDFTVPFMQLGVSILAYKSPHVEKTLDAYLAPFGGEVWIWILISVFVMTFLKTIVARISKMDWENPHP YGSFNKTTNSTSGMLKEIVEGRADLAITDLTITSEREEVIDFSIPFMNLGIAILYVKPQKAPPALFSFMDPFSSEVWLYLGIAYLGVSLCFFIIGRLSPIEWDNPYP YGSYNKSTNESTGMLREIMTGRADLAITDLTITSEREQALDFTIPFMNLGIAILYLKPQKATPELFTFMDPFSEEVWWFLGFSFLGVSLSFFILGRLSPSEWDNPYP YGVPEANG - TWTGMVGELIARKADLAVAGLTITAEREKVIDFSKPFMTLGISILYRVHMGRKPGYFSFLDPFSPGVWLFMLLAYLAVSCVLFLVARLTPYEWYSPHP

TM2

TM3

AIQ - DPDELENI $\overline{W N V N N S T W L M V G S I M Q Q G C D I L P R G P H M R I T T G M W W F F A L M M L S T Y T A N L A A F L T S N K W Q S S I K S L Q D L I E ~-~ Q D K V H F G S M R G G S T S L F F S E S N D ~}$ NVN - REMERQNIWHLSNALWLVLGSMLNQGCDLLPRGLPMRLLTAFWWIFALLISQTYIAKLAAFITSSKIAGDIGSLHDLVD - QNKVQFGTIRGGATSVYFSESND CNR - DPEVLENQWRIHNTGWLTVASIMTAGCDILPRSPQVRMFEATWWIFAIIIANSYTANLAAFLTSSKMEGSIANLKDLSA-QKKVKFGTIYGGSTYNLLADSNE CIE-EPEELENQFTINNSLWFTTGALLQQGSEIAPKALSTRTISAIWWFFTLIMVSSYTANLAAFLTIENPTSPINSVKDLADNKDDVQYGAKRTGSTRNFFSTSEE CIE - EPEELENQFTLGNSIWFTTGALLQQGSEIGPKALSTRTVASFWWFFTLIVVSSYTANLAAFLTIEKPQSLINSVDDLADNKDGVVYGAKKTGSTRNFFMTSAE CAQGRCNLLVNQYSLGNSLWFPVGGFMQQGSTIAPRALSTRCVSGVWWAFTLIIISSYTANLAAFLTVQRMDVPIESVDDLAD - QTAIEYGTIHGGSSMTFFQNSRY

TDYQRAWNQMKDFNPSAFTSTNKEGVARVRKEKGGYAFLMETTSLTYNIERNCDLTQIGEQIGEKHYGLAVPLGSDYRTNLSVSILQLSERGELQKMKNKWWKNHNV TDNRMAWNKMLSFKPDAFTKNNEEGVDRVKLSKGTYAFLMETTNLQYYVQRNCELTQIGESFGEKHYGIAVPLNADFRSNLSVGILRLSERGELFKLRNKWFNSNES TVYRLAFNLMNNDDPSAYTKDNLEGVDRVRKNRGDYMFLMETTTLEYHREQNCDLRSVGEKFGEKHYAIAVPFGAEYRSNLSVAILKLSERGELYDLKQKWWKNPNA PIYIKMNEYLNA - HPEMLMENNOOGVDKVKSG - TKYAFLMESTSIEFNTVRECNLTKVGDPLDEKGYGIAMVKNWPYRDKFNKALLELQEOGVLARLKNKWWNEVGA ERYKKMNKFMSE - NPQYLTEDNMEGVNRVKTN - THYAFLMESTSIEYNTKRECNLKKIGDALDEKGYGIAMRKDWPHRGKFNNALLELQEQGVLEKMKNKWWNEVGT QTYQRMWNYMYSKQPSVFVKSTEEGIARVLNS - - NYAFLLESTMNEYYRQRNCNLTQIGGLLDTKGYGIGMPVGSVFRDEFDLAILQLQENNRLEILKRKWWEGGKC

TM4

- TCDS - YHEVDGDELSIIELGGVFLVLAGGVLIGVILGIFEFLWNVQNVAVEERVTPWQAFKAELIFALKFWVRKKPMRISS - - SSDKSSSRRSS - - - - GSRRSSK - TCDSNVPTIDDGQFDMDSVGGLFVVLIVGVVVGLVIGVAEFLWHVQRISVKEKIPPMLALKAEFYFVIRFWLTRKPLHTYR - QSRDSTSTGYSSLEQITSASSAK - SCFEEPDPDATPDMTFEELRGIFYTLYAGILIAFLIGITEFLVYVQQVALEERLTFKDAFKKEIRFVLCVWNNRKPIVAGTPISSVRTTPRRSLDKSLDRTPKSSR GVCSAKSDDDGPSELGVDNLSGIYVVLVIGSIISIIISILCWCYFVYKKAKNYEVPFCDALAEEFRIVIRFSENERPLKSAQSIYSRSRNSSQSIESLKTDSEENMP GICATKEDAPDATPLDMNNLEGVFFVLLVGSCCALLYGIISWVLFVMKKAHHYRVPLRDALKEEFQFVIDFNNYVRVLKNSASIYSRSRQSSMSVASVAQESQ - - - - - - - PKEEDHRAKGLGMENIGGIFVVLICGLIVAIFMAMLEFLWTLR - HSEATEVSVCQEMVTELRSIILCQDSIHPRRRRAAVPPPRPPIPEERRPRGTATLSNGK 
(Figure 1-5a cont.)

EKSRSKTVS- - - - - - - - - - - - - - - - - - - - - - - - - - - - - - - -

KKKKTRRIEK $-\ldots$

RVVIGRSSEEMREMAQGSGSSSGSNNAGRGEKEARV - -

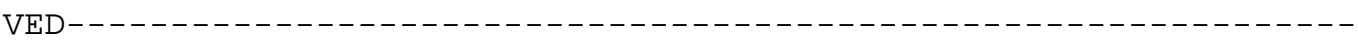

LCGAGEPDQLAQRLAQEAALVARGCTHIRVCPECRRFQGLRARPSPARSEESLEWEKTTNSSEPE 
(Figure 1-5a cont.)

GluR-IIA

GluR-IIB

GluR-IIC

GluR-IID

GluR-IIE

KA2

- - - - - - - - - MRLCPVVIYAFIIIIGFLEGIIALGGDDRNEITVGAIFYENEKE - - - IELSFDQAFREVN - - NMKFSELRFVTIKRYMPTNDSFLLQQI

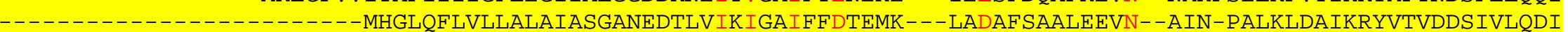
MKKELSGNSSARWPQMFGGNSREAGMWQRILLLGCMWSAFFMCRSRGQQINIGAFFYDDELE - - - LEKEFMTVVNAINGPESEQTMRFYPLIKRLKPEDGSVTMQEH - - - - - - - MHFCWIS - - LIILSLSRVQAQFYGGNAYEASSGQSIRLGLITDDATDR - - - IRQTFEHAISVVN - - - NELGVPLVGETEQVAYGNSVQAFAQ - - - - - - MFFNHFVILWSLFSIHISVNWAQYENFGG - YDNYQSLESVPIGLLTDQNTEQ - - - MNIVFDHAIDVAN - - - Q QEVGTSLTSLKEEVNYGDAYQSYGK - - - - - - - - - - - MPAELLLLLIVAFASPSCQVLSSLRMAAILDDQTVCGRGERLALALAREQINGIIEVPAKARVEVDIFELQRDSQYETTDT

TCELISNGVAAIFGPSSK-AASDIVAQIANATGIPHIEYDLKLEATRQEQLNHQMSINVAPSLSVLSRAYFEIIKSN - YEWRTFTLIYETPEG - - LARLQDLMNIQA SCDLIGSGVAAIFGPSSK-TNSDIVEVLCNMTGIPHLQFDWHPQQSNRERMNHQLTVNVAPMELFLSAAFSDILASKTFDWKSFTIAYERSSH - - LIRLQHILAWKQ ACDLIDNGVAAIFGPSSK-AASDIVALVCNSTGIPHIEFDISDEGIQAEKPNHQMTLNLYPAQAILSKAYADIVQNF - - GWRKFTIVYDADDARAAARLQDLLQLRE LCRLMQSGVGAVFGPAAR - HTASHLLNACDSKDIPFIYPHLSWGSNPDG - - - - - FNLHPSPEDIANALYDIVNQF - - EWSRFIFCYESAEY - - LKILDHLMTRYG LCRMLETGIAGVFGPSSR - HTAVHLMSICDAMDIPHIYSYMS - - ENAEG - - - - - - FNLHPHPADLAKALYSLITEF - - NWTRFIFLYESAEY - - LNILNELTTMLG MCQILPKGVVSVLGPSSSPASASTVSHICGEKEIPHIKVGPE - - ETPRLQYLRFASVSLYPSNEDVSLAVSRILKSF - - NYPSASLICAKAEC - - LLRLEELVRGFL

LNSDYVKLRNLAD - YADDYRILWKETDETFHEQRIILDCEPKTLKELLKVSIDFKLQGPFRNWFLTHLDTHNSGLRDIYN - - EDFKANITSVRLKVVDANPFERKKT LHKAGIKMQEFE - - RGDDYRILWKRINN - AREKFVLLDCPSDILVDVINASIGYNMTGSFNHLFLTNLDTHLSGIDGFYS - - RDFTVAVAAVRIRTYVPPPVHDEID VHNDVVRVRKFH - KDDDFRVMWKSIRG - - ERRVVLDCEPNMLVELLNSSTEFGLTGQYNHIFLTNLETYTDHLEELAADNETFAVNITAARLLVNPDPPPYSLPY IKGPVIKVMRYDLNLNGNYKSVLRRIRK-SEDSRIVVVGSTTGVAELLRQAQQVGIMNEDYTYIIGNLNLHTFDLEEYKYS - - - EANITGIRMFSPDQEEVRDLME KSGTVITVLRYDMQLNGNYKQVLRRVRK-SVDNRIVVVGSSETMPEFLNQAQQVGI INEDYKYI IGNLDFHSFDLEEYKYS - - - EANITGLRLFSPEKMAVKELLM ISKETLSVRMLDD - - SRDPTPLLKEIRD - DKVSTIIIDANASISHLILRKASELGMTSAFYKYILTTMDFPILHLDGIVED - - - - SSNILGFSMFNTSHPFYPEFVR

RLT - KVDQILGN - - - - - QTMLPILIYDAVVLFASSARNVIAAMQP - FHPP - - - NRHCGSSSPWMLGAFIVNEMKTISEDDVEPHFKTENMKLDEYGQRIHFNLEI VFDNSVDTRFS - - - - - - SLGSQLVYDSIVLFYN - ALLEISQRPG - FYIP - - - NFSCG - RGFWQPGPRLVEQMKQITPKMVKPPFKTQRLQINADGQREDFNLEV GYVTQRDNIVYESSD - PPRTLIHDL IHDALQLFAQSWRNASFFYPDRMVVPRITCDFAASGGRTWAMGRYLARLMKGTSG - VNNTNFRTSILQFDEDGQRITFNIEV KLHQELGESEPVNSGSTFITMEMALTYDAVRVIAETTKHLPYQPQM - - - - - - LNCSERHDNVQPDGSTFRNYMR - - SLEIKEKTITGRIYFEGNVRKGFTFDVIE KLGYPTDQDE - FRNGSCPITVEMALTYDAVQLFAQTLKNLPFKPMP - - - - - - - QNCSQRTESVRDDGSSFKNYMR - - TLRLTDRLLTGPIYFEGNVRKGYHLDVIE SLNMSWRENCEASTYLG - PALSAALMFDAVHVVVSAVRELNRSQEIG - - - - - VKPLACTSANIWPHGTSLMNYLR - - - - - MVEYDGLTGRVEFNSKGQRTNYTLRI

YKPT - - VNEPMMVWTPDN - - - GIKKRLLN - - - LELESAGTTQDFSEQRKVYTVVTHYE - EPYFMMKEDHE - - NFRGREKYEGYAVDLISKLSELMEFDYEFMIVNG YNPI - - IDRVTHIWNKEFQLVDFEKLRENSTQALKQKRLQNKEDFSQKPIRYTVATRVG - KPYFSWREEPEGVHYEGNERFEGYAVDLIYMLAQECKFDFNFEPVRD YDPL - - DGIGIAIWDPRG - - - - - - - - - - - QITQLNVDVKAQKKMIYRVATRIG - PPYFSYNETARELNLTGNALYQGYAVDLIDAIARHVGFEYVFVPVAD LQTS - - GLVKVGTWEEGK - - - - - DFEFQR - PPQAVNFNDIDDGSLVNKTFIVLISVATKPYASLVESID - - TLIGNNQFQGYGVDLIKELADKLGFNFTFR - DGG LQPS - - GIVKVGTWDEDR - - - - - QYRPQRLAPTTAQFDSVDN-SLANKTFI ILLSVPNKPYAQLVETYK- - QLEGNSQYEGYGVDL IKELADKLGFNFTFV-NGG LEKSRQGHREIGVWYSNR - - - - - - - - - TLAMNATTLDINLSQTLANKTLVVTTILENPYVMRRPNFQ - -ALSGNERFEGFCVDMLRELAELLRFRYRLRLVED 
(Figure 1-5a cont.)

TM1

N - - GKYNPETKQWDGI IRKLIDHHAQIGVCDLTITQMRRSVVDFTVPFMQLGISILHYKSPPEPKNQFAFLEPFAVEVWIYMIFAQLIMTLAFVFIARLSYREWLPP NKYGSYDANTDEWDGI IRQLIDNNAQIGICDLTITQARRSVVDFTVPFMQLGISILSYKEPPPKADIYAFLNPYNAEVWLFVMIAMMITAFALIFTGRIDQYEWDQP QQYGKLDKETKQWNGIIGEIINNDAHMGICDLTITQARKTAVDFTVPFMQLGVSILAYKSPHVEKTLDAYLAPFGGEVWIWILISVFVMTFLKTIVARISKMDWENP NDYGSFNKTTNSTSGMLKEIVEGRADLAITDLTITSEREEVIDFSIPFMNLGIAILYVKPQKAPPALFSFMDPFSSEVWLYLGIAYLGVSLCFFIIGRLSPIEWDNP NDYGSYNKSTNESTGMLREIMTGRADLAITDLTITSEREQALDFTIPFMNLGIAILYLKPQKATPELFTFMDPFSEEVWWFLGFSFLGVSLSFFILGRLSPSEWDNP GLYGAPEPNG - SWTGMVGELINRKADLAVAAFTITAEREKVIDFSKPFMTLGISILYRVHMGRKPGYFSFLDPFSPAVWLFMLLAYLAVSCVLFLAARLSPYEWYNP

\section{TM2}

TM3

NPAIQD - PDELENI $\overline{W N V N N S T W L M V G S I M Q Q G C D I L P R G P H M R I ~} \overline{L T G M W W F F A L M M L S T Y T A N L A A F L T S N K W Q S S I K S L Q D L I E ~-~ Q D K V H F G S M R G G S T S L F F S E S ~}$ VENVNR-EMERQNIWHLSNALWLVLGSMLNQGCDLLPRGLPMRLLTAFWWIFALLISQTYIAKLAAFITSSKIAGDIGSLHDLVD - QNKVQFGTIRGGATSVYFSES HPCNRD -PEVLENQWRIHNTGWLTVASIMTAGCDILPRSPQVRMFEATWWIFAIIIANSYTANLAAFLTSSKMEGSIANLKDLSA-QKKVKFGTIYGGSTYNLLADS YPCIEE - PEELENQFTINNSLWFTTGALLQQGSEIAPKALSTRTISAIWWFFTLIMVSSYTANLAAFLTIENPTSPINSVKDLADNKDDVQYGAKRTGSTRNFFSTS YPCIEE - PEELENQFTLGNSIWFTTGALLQQGSEIGPKALSTRTVASFWWFFTLIVVSSYTANLAAFLTIEKPQSLINSVDDLADNKDGVVYGAKKTGSTRNFFMTS HPCLRARPHILENQYTLGNSLWFPVGGFMQQGSEIMPRALSTRCVSGVWWAFTLIIISSYTANLAAFLTVQRMEVPVESADDLAD - QTNIEYGTIHAGSTMTFFQNS

NDTDYQRAWNQMKDFNPSAFTSTNKEGVARVRKEKGGYAFLMETTSLTYNIERNCDLTQIGEQIGEKHYGLAVPLGSDYRTNLSVSILQLSERGELQKMKNKWWKNH NDTDNRMAWNKMLSFKPDAFTKNNEEGVDRVKLSKGTYAFLMETTNLQYYVQRNCELTQIGESFGEKHYGIAVPLNADFRSNLSVGILRLSERGELFKLRNKWFNSN NETVYRLAFNLMNNDDPSAYTKDNLEGVDRVRKNRGDYMFLMETTTLEYHREQNCDLRSVGEKFGEKHYAIAVPFGAEYRSNLSVAILKLSERGELYDLKQKWWKNP EEPIYIKMNEYLNA-HPEMLMENNOOGVDKVKSG - TKYAFLMESTSIEFNTVRECNLTKVGDPLDEKGYGIAMVKNWPYRDKFNKALLELOEOGVLARLKNKWWNEV AEERYKKMNKFMSE - NPQYLTEDNMEGVNRVKTN - THYAFLMESTSIEYNTKRECNLKKIGDALDEKGYGIAMRKDWPHRGKFNNALLELQEQGVLEKMKNKWWNEV RYQTYQRMWNYMQSKQPSVFVKSTEEGIARVLNS - -RYAFLLESTMNEYHRRLNCNLTQIGGLLDTKGYGIGMPLGSPFRDEITLAILQLQENNRLEILKRKWWEGG

\section{TM4}

NV - TCDS - YHEVDGDELSI IELGGVFLVLAGGVLIGVILGIFEFLWNVQNVAVEERVTPWQAFKAELIFALKFWVRKKPMRISS - - SSDKSSSRRSS - - - - GSRRS ES-TCDSNVPTIDDGQFDMDSVGGLFVVLIVGVVVGLVIGVAEFLWHVQRISVKEKIPPMLALKAEFYFVIRFWLTRKPLHTYR- -QSRDSTSTGYSSLEQITSASS NA-SCFEEPDPDATPDMTFEELRGIFYTLYAGILIAFLIGITEFLVYVQQVALEERLTFKDAFKKEIRFVLCVWNNRKPIVAGTPISSVRTTPRRSLDKSLDRTPKS GAGVCSAKSDDDGPSELGVDNLSGIYVVLVIGSIISIIISILCWCYFVYKKAKNYEVPFCDALAEEFRIVIRFSENERPLKSAQSIYSRSRNSSQSIESLKTDSEEN GTGICATKEDAPDATPLDMNNLEGVFFVLLVGSCCALLYGIISWVLFVMKKAHHYRVPLRDALKEEFQFVIDFNNYVRVLKNSASIYSRSRQSSMSVASVAQESQ - RC - - - PKEEDHRAKGLGMENIGGIFIVLICGLIIAVFVAVMEFIWSTRRSAESEEVSVCQEMLQELRHAVSCRKTSRSRRRRRPGGPSRALLSLRAVREMRLSNGK 
(Figure 1-5a cont.)

SKEKSRSKTVS- - - - - - - - - - - - - - - - - - - - - - - - - - - - - - - - - - - - - - - - - - - - - - - - - - - AKKKKKTRRIEK- - - -

SRRVVIGRSSEEMREMAQGSGSSSGSNNAGRGEKEARV- $-\cdots$

MPVED-1- - - - - - - - - - -

LYSAGAGGDAGSAHGGPQRLLDDPGPPSGARPAAPTPCTHVRVCQECRRIQALRASGAGAPPRGLGVPAEATSPPRPRPGPAGPRELAEHE 
- - - - MRLCPVVIYAFIIIIGFLEGIIALGGDDRNEITVGAIFYENEKE - - - - IELSFDQAFREVNN-- MKFSELRFVTIKRYMPTNDSFLLQQITCELISNGVAAI

FGPSSKAASDIVAQIANATGIPHIEYDLKLEATRQEQLNHQMSINVAPSLSVLSRAYFEIIKSNYEWRTFTLIYETPEGLARLQDLMNIQALNSDYVKLRNLADYAD FGPSHSSSVSAVQSICNALEVPHIQTRWKHPSVDNK - - DLFYINLYPDYAAISRAILDLVLY - YNWKTVTVVYEDSTGLIRLQELIKAPSRYNIKIKIRQLPSGNK

DYRILWKETDETFHEQRIILDCEPKTLKELLKVSIDFKLQGPFRNWFLTHLDTHNSGLRDIYNEDFKANITSVRLKVVD - - - - ANPFERKKTRLTKVDQILGNQTML DAKPLLKEMKK-GKEFYVIFDCSHETAAEILKQILFMGMMTEYYHYFFTTLDLFALDLELYR - - YSGVNMTGFRLLNIDNPHVSSIIEKWSMERLQAPPRPETGLLD

PILIYDAVVLFASSARNVIAAMQP - - FHPPNRHCGSSSPWMLGAFIVNEMKTISEDDVEPHFKTENMKLDEYGQRIHFNLEIYKPTVNEPMMVWTPDNGIKKRLLNL GMMTTEAALMYDAVYMVAIASHRASQLTVSSLQCHRHKPWRLGPRFMNLIKEARWDGLTGHITFN - - KTNGLRKDFDLDI ISLKEEGTEKIGIWNSNSGLNMTDSNK

ELESAGTTQDFSEQRKVYTVVTHYEEPYFMMKEDHENFRGREKYEGYAVDLISKLSELMEFDYEFMIVNGNGKYNP - - ETKQWDGI IRKLIDHHAQIGVCDLTITQM DKSSN - - - ITDSLANRTLIVTTILEEPYVMYRKSDKPLYGNDRFEGYCLDLLKELSNILGFIYDVKLVP - DGKYGAQNDKGEWNGMVKELIDHRADLAVAPLTITYV

TM2

RRSVVDFTVPFMQLGISILHYKSPPEPKNQFAFLEPFAVEVWIYMIFAQLIMTLAFVFIARLSYREWLPPNPAIQDPDELENIWNVNNSTWLMVGSIMQQGCDILPR REKVIDFSKPFMTLGISILYRKPNGTNPGVFSFLNPLSPDIWMYVLLACLGVSCVLFVIARFTPYEWYNPHPCNPDSDVVENNFTLLNSFWFGVGALMQQGSELMPK

\section{TM3}

GPHMRI LTGMWWFFALMMLSTYTANLAAFLTSNKWOSSIKSLODLIEODKVHFGSMRGGSTSLFFSESNDTDYORAWNQMKDFNPSAFTSTNKEGVARVRKEKGGYA ALSTRIVGGIWWFFTLIIISSYTANLAAFLTVERMESPIDSADDLAKQTKIEYGAVRDGSTMTFFKKSKISTYEKMWAFMSSRQQTALVRNSDEGIQRVLTT - - DYA

TM4

FLMETTSLTYNIERNCDLTQIGEQIGEKHYGLAVPLGSDYRTNLSVSILQLSERGELQKMKNKWWKNHNVTCDSYHEVDGDELSIIELGGVFLVLAGGVLIGVILGI LLMESTSIEYVTQRNCNLTQIGGLIDSKGYGVGTPIGSPYRDKITIAILQLQEEGKLHMMKEKWWRGN - - GCPEEDNKEASALGVENIGGIFIVLAAGLVLSVFVAI

FEFLWNVQNVAVEERVTPWQAFKAELIFALKFWVRKKPMRISSSSDKSSSRRSSGSRRSSKEKSRSKTVS GEFIYKSRKNNDIEQCLSFNAIMEELGISLKNQKKIKKKSRTKGKSSFTSILTCHQRRTQRKETVA - - - -

Figure 1-5b. Sequence alignment with predicted amino acid sequences of the postsynaptic receptor subunits at Drosophila NMJ (GluRIIA, GluRIIB, GluRIIC, GluRIID and GluRIIE) and human's kainate receptor subunits (GluR5, GluR6, GluR7, KA1 and KA2). Sequence comparisons are achieved to investigate the similarities of the each subunit of Drosophila NMJ and human. Since homologous sequences are different lengths, gaps are inserted to gain the best match. Clustal W2 is used for sequence analysis. 
(Figure 1-5b cont.)

GluR-IIB MHGLQFLVLLALAIASG - - - - - - - - ANEDTLVIKIGAIFFDTEMKLAD - - - AFSAALEEVN - - AINPALKLDAIKRYVTVDDSIVLQDISCDLIGSGVAAI

FGPSSKTNSDIVEVLCNMTGIPHLQFDWHPQQSNRERMNHQLTVNVAPMELFLSAAFSDILASKTFDWKSFTIAYERSSHLIRLQHILAWKQLHKAGIKMQEFERGD FGPSHSSSVSAVQSICNALEVPHIQTRWKHPSVDNK - - DLFYINLYPDYAAISRAILDLVLY - YNWKTVTVVYEDSTGLIRLQELIKAPSRYNIKIKIRQLPSGN

- DYRILWKRINNAREKFVLLDCPSDILVDVINASIGYNMTGSFNHLFLTNLDTHLSGIDGF - YSRDFTVAVAAVRIRTYVPPPVHDEIDVFDNSVDTRFSS - LGSQL KDAKPLLKEMKKGKEFYVIFDCSHETAAEILKQILFMGMMTEYYHYFFTTLDLFALDLELYRYSGVNMTGFRLLNIDNPHVSSIIEKWSMERLQAPPRPETGLLDGM

VYDSIVLFYNALLEISQRPG - - - FYIPNFSCGRG - FWQPGPRLVEQMKQITPKMVKPPFKTQRLQINADGQREDFNLEVYNPIIDRVTHIWNKEFQLVDFEKLREN MTTEAALMYDAVYMVAIASHRASQLTVSSLQCHRHKPWRLGPRFMNLIKEARWDGLTGHITFN - - - - KTNGLRKDFDLDIISLKEEGTEKIG - - - - - IWNSNSGLN

STOALKOKRLONKEDFSOKPIRYTVATRVGKPYFSWREEPEGVHYEGNERFEGYAVDLIYMLAOECKFDFNFEPVRDNKYGSYDANTDEWDGIIROLIDNNAOIGIC MTDSNKDKSSNITDSLANR - - TLIVTTILEEPYVMYRKSDK - - PLYGNDRFEGYCLDLLKELSNILGFIYDVKLVPDGKYGAQND - KGEWNGMVKELIDHRADLAVA

TM1

TM2

DLTITQARRSVVDFTVPFMQLGISILSYKEPPPKADIYAFLNPYNAEVWLFVMIAMMITAFALIFTGRIDQYEWDQPVENVNREMERQNIWHLSNALWLVLGSMLNQ PLTITYVREKVIDFSKPFMTLGISILYRKPNGTNPGVFSFLNPLSPDIWMYVLLACLGVSCVLFVIARFTPYEWYNPHPCNPDSDVVENNFTLLNSFWFGVGALMQQ

\section{TM3}

GCDLLPRGLPMRLLTAFWWIFALLISQTYIAKLAAFITSSKIAGDIGSLHDLVDONKVOFGTIRGGATSVYFSESNDTDNRMAWNKMLSFKPDAFTKNNEEGVDRVK GSELMPKALSTRIVGGIWWFFTLIIISSYTANLAAFLTVERMESPIDSADDLAKQTKIEYGAVRDGSTMTFFKKSKISTYEKMWAFMSSRQQTALVRNSDEGIQRVL

\section{TM4}

LSKGTYAFLMETTNLQYYVQRNCELTQIGESFGEKHYGIAVPLNADFRSNLSVGILRLSERGELFKLRNKWFNSNESTCDSNVPTIDDGQFDMDSVGGLFVVLIVGV TTD - -YALLMESTSIEYVTQRNCNLTQIGGLIDSKGYGVGTPIGSPYRDKITIAILQLQEEGKLHMMKEKWWRGNGCPEEDNK - - EASALGVENIGGIFIVLAAGL

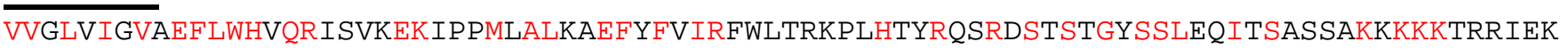
VLSVFVAIGEFIYKSRKNNDIEQCLSFNAIMEELGISLK- - - - - - - NQKKIKKKSRTKGKSSFTSILTCHQRRTQRKETVA- 
(Figure 1-5b cont.)

GluR-IIC MKKELSGNSSARWPQMFGGNSREAGMWQRILLLGCMWSAFFMCRSRGQQIN - IGAFFYDDELE - - - LEKEFMTVVNAINGPESEQTMRFYPLIKRLKPEDGSVTMQ GluR-5 - - - - MEHGTLLAQPGLWTRDTSWALLYFLCYILPQTAPQVLRIGGIFETVENEPVNVEELAFKFAVTSINRNRTLMPNTTLTYDIQRINLFDSFEAS

EHACDLIDNGVAAIFGPSSKAASDIVALVCNSTGIPHIEFDISDEGIQAEKPNHQMTLNLYPAQAILSKAYADIVQNFGWRKFTIVYDADDARAAARLQDLLQLREV RRACDQLALGVAALFGPSHSSSVSAVQSICNALEVPHIQTRWKHPSVDNKD - - - LFYINLYPDYAAISRAILDLVLYYNWKTVTVVYEDSTG - - LIRLQELIKAPSR

HNDVVRVRKFHK - DDDFRVMWKSIRGER - RVVLDCEPNMLVELLNSSTEFGLTGQYNHIFLTNLETYTDHLEELAADNETFAVNITAARLLVNPDPPPYSLPYGYV YNIKIKIRQLPSGNKDAKPLLKEMKKGKEFYVIFDCSHETAAEILKQILFMGMMTEYYHYFFTTLDLFALDLELYRYS - - - - GVNMTGFRLLNIDNPHVSSIIEKWS

TQRDNIVYESSDPPRTLIHDLIHDALQLFAQSWRNASFFYPDRMVVPRITCDFAASGGRTWAMGRYLARLMKGTSGVNNTNFRTSILQFDEDGQRITFNIEVYDPLD MER - - - LQAPPRPETGLLDGMMTTEAALMYDAVYMVAIAS - - HRASQLTVSSLQCHRHKPWRLGPRFMNLIKEAR - - WDGLTGHITFNKTNGLRKDFDLDIISLKE

G - - IGIAIWDPRGQITQLNVDVKAQKKMIYRVATR - - - - - - IGPPYFSYNETARELNLTGNALYOGYAVDLIDAIARHVGFEYVFVPVADQOYGKLDKETKOWNGI EGTEKIGIWNSNSGLNMTDSNKDKSSNITDSLANRTLIVTTILEEPYVMYRKSDKPL - -YGNDRFEGYCLDLLKELSNILGFIYDVKLVPDGKYG - AQNDKGEWNGM

TM1

IGEI INNDAHMGICDLTITQARKTAVDFTVPFMQLGVSILAYKSPHVEKTLDAYLAPFGGEVWIWILISVFVMTFLKTIVARISKMDWENPHPCNRDPEVLENQWRI VKELIDHRADLAVAPLTITYVREKVIDFSKPFMTLGISILYRKPNGTNPGVFSFLNPLSPDIWMYVLLACLGVSCVLFVIARFTPYEWYNPHPCNPDSDVVENNFTL

TM2

TM3

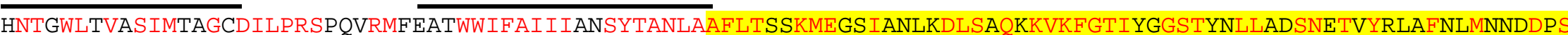
LNSFWFGVGALMQQGSELMPKALSTRIVGGIWWFFTLIIISSYTANLAAFLTVERMESPIDSADDLAKQTKIEYGAVRDGSTMTFFKKSKISTYEKMWAFMSSRQQT

AYTKDNLEGVDRVRKNRGDYMFLMETTTLEYHREQNCDLRSVGEKFGEKHYAIAVPFGAEYRSNLSVAILKLSERGELYDLKQKWWKNPNASCFEEPDPDATPDMTF ALVRNSDEGIQRVLTT - - DYALLMESTSIEYVTQRNCNLTQIGGLIDSKGYGVGTPIGSPYRDKITIAILQLQEEGKLHMMKEKWWRG - - NGCPEEDNKEAS - ALGV

\section{TM4}

EELRGIFYTLYAGILIAFLIGITEFLVYVQQVALEERLTFKDAFKKEIRFVLCVWNNRKPIVAGTPISSVRTTPRRSLDKSLDRTPKSSRRVVIGRSSEEMREMAQG ENIGGIFIVLAAGLVLSVFVAIGEFIYKSRKNNDIEQCLSFNAIMEELGISLKNQKKIK - - - - - - - KKSRTKGKSSFTSILTCHQRRTQRKETVA - - . - . - . - . 
(Figure 1-5b cont.)

GluR-IID - - -MHFCWISLIILSLSRVQAQFYGGNAYEASSGQSIRLG - - - LITDDATDRIRQTFEHAISVVNN - - -ELGVPLVGETEQVAYGNSVQAFAQLCRLMQSGVGAV GluR-5 MEHGTLLAQPGLWTRDTSWALLYFLCYILPQTAPQVLRIGGIFETVENEPVNVEELAFKFAVTSINRNRTLMPNTTLTYDIQRINLFDSFEASRRACDQLALGVAAL

FGPAARHTASHLLNACDSKDIPFIYPHLSWGSNPDG - - - FNLHPSPEDIANALYDIVNQFEWSRFIFCYESAEYLKILDHLMTRYGIKGPVIKVMRYDLNLNGNYK FGPSHSSSVSAVQSICNALEVPHIQTRWKHPSVDNKDLFYINLYPDYAAISRAILDLVLYYNWKTVTVVYEDSTGLIRLQELIKAPSRYNIKIKIRQLPS-GNKDAK

SVLRRIRKSEDSRIVVVGSTTGVAELLRQAQQVGIMNEDYTYIIGNLNLHTFDLEEYKYSEANITGIRMFSPDQEEVRDLMEKLHQELGESEPVNSG - - - STFITME PLLKEMKKGKEFYVIFDCSHETAAEILKQILFMGMMTEYYHYFFTTLDLFALDLELYRYSGVNMTGFRLLNIDNPHVSSIIEKWSMERLQAPPRPETGLLDGMMTTE

MALTYDAVRVIAETTKHLPYQPQMLNCSERHDNVQPDGSTFRNYMRSLEIKEKTITGRIYFEGNVRKGFTFDVIELQTSGLVKVGTWEEGKDFEFQRPPQAVNFNDI AALMYDAVYMVAIASHRASQLTVSSLQCHRHKPWR - LGPRFMNLIKEARWDGLTGHITFNKTNGLRKDFDLDIISLKEEGTEKIGIWNSNSGLNMTDSNKDKSSNIT

DDGSLVNKTFIVLISVATKPYASLVESIDTLIGNNOFOGYGVDLIKELADKLGFNFTFRDGGNDYGSFNKTTNSTSGMLKEIVEGRADLAITDLTITSEREEVIDFS D - - SLANRTLIVTT - ILEEPYVMYRKSDKPLYGNDRFEGYCLDLLKELSNILGFIYDVKLVPDGKYGAQNDKGEWNGMVKELIDHRADLAVAPLTITYVREKVIDFS

TM1

\section{TM2}

IPFMNLGIAILYVKPQKAPPALFSFMDPFSSEVWLYLGIAYLGVSLCFFIIGRLSPIEWDNPYPCIEEPEELENQFTINNSLWFTTGALLQQGSEIAPKALSTRTIS KPFMTLGISILYRKPNGTNPGVFSFLNPLSPDIWMYVLLACLGVSCVLFVIARFTPYEWYNPHPCNPDSDVVENNFTLLNSFWFGVGALMQQGSELMPKALSTRIVG

TM3

AIWWFFTLIMVSSYTANLAAFLTIENPTSPINSVKDLADNKDDVOYGAKRTGSTRNFFSTSEEPIYIKMNEYLN - AHPEMLMENNOOGVDKVKSGTKYAFLMESTSI GIWWFFTLIIISSYTANLAAFLTVERMESPIDSADDLAKQT - KIEYGAVRDGSTMTFFKKSKISTYEKMWAFMSSRQQTALVRNSDEGIQRVLT - TDYALLMESTSI

TM4

EFNTVRECNLTKVGDPLDEKGYGIAMVKNWPYRDKFNKALLELQEQGVLARLKNKWWNEVGAGVCSAKSDDDGPSELGVDNLSGIYVVLVIGSIISIIISILCWCYF EYVTQRNCNLTQIGGLIDSKGYGVGTPIGSPYRDKITIAILQLQEEGKLHMMKEKWWRGNGC - - - PEEDNKEASALGVENIGGIFIVLAAGLVLSVFVAIGEFIYK

VYKKAKNYEVPFCDALAEEFRIVIRFSENERPLKSAQSIYSRSRNSSQSIESLKTDSEENMPVED SRKNNDIEQCLSFNAIMEELGISLKNQKKIK--KKSRTKGKSSFTSILTCHQRRTQRKETVA - - - 
(Figure 1-5b cont.)

GluR-IIE MFFNHFVILWSLFSIHISVNWAQYENFGGYDNYQSLESVPIG - - - LLTDQNTEQMNIVFDHAIDVAN - - - QEVGTSLTSLKEEVNYGDAYQSYGKLCRMLETGIA

GVFGPSSRHTAVHLMSICDAMDIPHIYSYMSENAEG - - - - - FNLHPHPADLAKALYSLITEFNWTRFIFLYESAEYLNILNELTTMLGKSGTVITVLRYDMQLNGN ALFGPSHSSSVSAVQSICNALEVPHIQTRWKHPSVDNKDLFYINLYPDYAAISRAILDLVLYYNWKTVTVVYEDSTGLIRLQELIKAPSRYNIKIKIRQLPSGNK-D

YKQVLRRVRKSVDNRIVVVGSSETMPEFLNQAQQVGIINEDYKYIIGNLDFHSFDLEEYKYSEANITGLRLFSPEKMAVKELLMKLGYPTDQDEFRNGS - - - CPIT AKPLLKEMKKGKEFYVIFDCSHETAAEILKQILFMGMMTEYYHYFFTTLDLFALDLELYRYSGVNMTGFRLLNIDNPHVSSIIEKWSMERLQAPPRPETGLLDGMMT

VEMALTYDAVQLFAQTLKNLPFKPMPQNCSQRTESVRDDGSSFKNYMRTLRLTDRLLTGPIYFEGN - -VRKGYHLDVIELQPSGIVKVGTWDEDRQYRPQRLAPTTA TEAALMYDAVYMVAIASHRASQLTVSSLQCHRHKPWR - LGPRFMNLIKEARWDG - - LTGHITFNKTNGLRKDFDLDIISLKEEGTEKIGIWNSNSGLN - -MTDSNKD

OFDSVDNSLANKTFIILLSVPNKPYAOLVETYKOLEGNSOYEGYGVDLIKELADKLGFNFTFVNGGNDYGSYNKSTNESTGMLREIMTGRADLAITDLTITSEREOA KSSNITDSLANRTLIVTT - ILEEPYVMYRKSDKPLYGNDRFEGYCLDLLKELSNILGFIYDVKLVPDGKYGAQNDKGEWNGMVKELIDHRADLAVAPLTITYVREKV

\section{TM2}

LDFTIPFMNLGIAILYLKPQKATPELFTFMDPFSEEVWWFLGFSFLGVSLSFFILGRLSPSEWDNPYPCIEEPEELENQFTLGNSIWFTTGALLQQGSEIGPKALST IDFSKPFMTLGISILYRKPNGTNPGVFSFLNPLSPDIWMYVLLACLGVSCVLFVIARFTPYEWYNPHPCNPDSDVVENNFTLLNSFWFGVGALMQQGSELMPKALST

TM3

RTVASFWWFFTLIVVSSYTANLAAFLTIEKPOSLINSVDDLADNKDGVVYGAKKTGSTRNFFMTSAEERYKKMNKFMSENPOYLTEDNMEGVNRVKTNTHYAFLMES RIVGGIWWFFTLIIISSYTANLAAFLTVERMESPIDSADDLAKQTK- IEYGAVRDGSTMTFFKKSKISTYEKMWAFMSSRQQTALVRNSDEGIQRVLTTDYALLMES

TM4

TSIEYNTKRECNLKKIGDALDEKGYGIAMRKDWPHRGKFNNALLELQEQGVLEKMKNKWWNEVGTGICATKEDAPDATPLDMNNLEGVFFVLLVGSCCALLYGIISW TSIEYVTQRNCNLTQIGGLIDSKGYGVGTPIGSPYRDKITIAILQLQEEGKLHMMKEKWWRGNG - - - - CPEEDNKEASALGVENIGGIFIVLAAGLVLSVFVAIGEF

VLFVMKKAHHYRVPLRDALKEEFQFVIDFNNYVRVLKNSASIYSRSRQSSMSVASVAQESQ--

IYKSRKNNDIEQCLSFNAIMEELGISLKNQKKIKKKSRTKGKSSFTSILTCHQRRTQRKETVA 
(Figure 1-5b cont.)

GluR-IIA

GluR-6

- - - -MRLCPVVIYAFIIIIGFLEGIIALGGDDRNEITVGAIFYENE - - - - - KEIELSFDQAFREVNNMKFSELRFVTIKRYMPTNDSFLLQQITCELISNGVAAIF MKIIFPILSNPVFRRTVKLLLCLLWIGYSQGTTHVLRFGGIFEYVESGPMGAEELAFRFAVNTINRNRTLLPNTTLTYDTQKINLYDSFEASKKACDQLSLGVAAIF

GPSSKAASDIVAQIANATGIPHIEYDLKLEATRQEQLNHQMSINVAPSLSVLSRAYFEIIKSNYEWRTFTLIYETPEGLARLQDLMNIQALNSDYVKLRNLADYADD GPSHSSSANAVQSICNALGVPHIQTRWKHQVSDNK - - DSFYVSLYPDFSSLSRAILDLVQF - FKWKTVTVVYDDSTGLIRLQELIKAPSRYNLRLKIRQLPADTKD

YRILWKETDETFHEQRI ILDCEPKTLKELLKVSIDFKLQGPFRNWFLTHLDTHNSGLRDIYNEDFKAN - ITSVRLKVVDANPFERKKTRLTKVDQILGNQTMLPILI AKPLLKEMKR - GKEFHVIFDCSHEMAAGILKQALAMGMMTEYYHYIFTTLDLFALDVEPYRYSGVNMTGFRILNTENTQVSSIIEKWSMERLQAPPKPDSGLLDGFM

YDAVVLFASSARNVIAAMQPFHPPNR - - HCGSSSPWMLGAFIVNEMKTISEDDVEPHFKTENMKLDEYGQRIHFNLEIYKPTVNEPMMVWTPDNGIKKRLLNLELE TTDAALMYDAVHVVSVAVQQFPQMTVSSLQCNRHKPWRFGTRFMSLIKEAHWEGLTGRITFN - KTNGLRTDFDLDVISLKEEGLEKIGTWDPASGLNMTES - QKGK

SAGTTQDFSEQRKVYTVVTHYEEPYFMMKEDHENFRGREKYEGYAVDLISKLSELMEFDYEFMIVNGN - - GKYNPETKQWDGIIRKLIDHHAQIGVCDLTITQMRRS PANITDSLSN - - RSLIVTTILEEPYVLFKKSDKPLYGNDRFEGYCIDLLRELSTILGFTYEIRLVEDGKYGAQDDANGQWNGMVRELIDHKADLAVAPLAITYVREK

TM2

VVDFTVPFMQLGISILHYKSPPEPKNOFAFLEPFAVEVWIYMIFAOLIMTLAFVFIARLSYREWLPPNPAIODPDELENIWNVNNSTWLMVGSIMOOGCDILPRGPH VIDFSKPFMTLGISILYRKPNGTNPGVFSFLNPLSPDIWMYILLAYLGVSCVLFVIARFSPYEWYNPHPCNPDSDVVENNFTLLNSFWFGVGALMQQGSELMPKALS

TM3

MRILTGMWWFFALMMLSTYTANLAAFLTSNKWOSSIKSLODLIEODKVHFGSMRGGSTSLFFSESNDTDYORAWNOMKDFNPSAFTSTNKEGVARVRKEKGGYAFLM TRIVGGIWWFFTLIIISSYTANLAAFLTVERMESPIDSADDLAKQTKIEYGAVEDGATMTFFKKSKISTYDKMWAFMSSRRQSVLVKSNEEGIQRVLTS - - DYAFLM

TM4

ETTSLTYNIERNCDLTQIGEQIGEKHYGLAVPLGSDYRTNLSVSILQLSERGELQKMKNKWWKNHNVTCDSYHEVDGDELSIIELGGVFLVLAGGVLIGVILGIFEF ESTTIEFVTQRNCNLTQIGGLIDSKGYGVGTPMGSPYRDKITIAILQLQEEGKLHMMKEKWWRGN - - GCPEEESKEASALGVQNIGGIFIVLAAGLVLSVFVAVGEF

LWNVQNVAVEERVTPWQAFKAELIFALKFWVRKKPMRISSSSDKSSSRRSSGSRRSSKEKSRSKTVS

LYKSKKNAQLE . . . . . . . . . . . . KESSIWLVPPYHPDTV . . . . . . . . . . . 
(Figure 1-5b cont.)

GluR-IIB

- - - - - - - - MHGLQFLVLLALAIASGANEDTLVIKIGAIFFDTEMKLADAFSAALEEVN - - - - - AINPALKLDAIKRYVTVDDSIVLQDISCDLIGSGVAAIF MKIIFPILSNPVFRRTVKLLLCLLWIGYSQGTTHVLRFGGIFEYVESGPMGAEELAFRFAVNTINRNRTLLPNTTLTYDTQKINLYDSFEASKKACDQLSLGVAAIF

GPSSKTNSDIVEVLCNMTGIPHLQFDWHPQQSNRERMNHQLTVNVAPMELFLSAAFSDILASKTFDWKSFTIAYERSSHLIRLQHILAWKQLHKAGIKMQEFERG - D GPSHSSSANAVQSICNALGVPHIQTRWKHQVSD - - - - NKDSFYVSLYPDFSSLSRAILDLVQFFKWKTVTVVYDDSTGLIRLQELIKAPSRYNLRLKIRQLPADTK

DYRILWKRINNAREKFVLLDCPSDILVDVINASIGYNMTGSFNHLFLTNLDTHLSGIDGFYSRDFTVAVAAVR - - IRTYVPPPVHDEIDVFDNSVDTRFSSLGSQLV DAKPLLKEMKRGKEFHVIFDCSHEMAAGILKQALAMGMMTEYYHYIFTTLDLFALDVEPYRYSGVNMTGFRILNTENTQVSSIIEKWSMERLQAPPKPDSGLLDGFM

YDSIVLFYNALLEIS - - - QRPGFYIPNFSCGRG - FWQPGPRLVEQMKQITPKMVKPPFKTQRLQINADGQREDFNLEVYNPIIDRVTHIWNKEFQLVDFEKLRENS TTDAALMYDAVHVVSVAVQQFPQMTVSSLQCNRHKPWRFGTRFMSLIKEAHWEGLTGRITFN - - - KTNGLRTDFDLDVISLKEEGLEKIG - - - - - - TWDPASGLNM

TQALKQKRLQNKEDFSQKPIRYTVATRVGKPYFSWREEPEGVHYEGNERFEGYAVDLIYMLAQECKFDFNFEPVRDNKYGSYDANTDEWDGIIRQLIDNNAQIGICD TESQKGKPANITDSLSNR - - SLIVTTILEEPYVLFKKSDK - - PLYGNDRFEGYCIDLLRELSTILGFTYEIRLVEDGKYGAQDDANGQWNGMVRELIDHKADLAVAP

TM1

TM2

LTITQARRSVVDFTVPFMQLGISILSYKEPPPKADIYAFLNPYNAEVWLFVMIAMMITAFALIFTGRIDQYEWDQPVENVNREMERQNIWHLSNALWLVLGSMLNQ LAITYVREKVIDFSKPFMTLGISILYRKPNGTNPGVFSFLNPLSPDIWMYILLAYLGVSCVLFVIARFSPYEWYNPHPCNPDSDVVENNFTLLNSFWFGVGALMQQG

TM3

CDLLPRGLPMRLLTAFWWIFALLISQTYIAKLAAFITSSKIAGDIGSLHDLVDQNKVQFGTIRGGATSVYFSESNDTDNRMAWNKMLSFKPDAFTKNNEEGVDRVKL SELMPKALSTRIVGGIWWFFTLIIISSYTANLAAFLTVERMESPIDSADDLAKQTKIEYGAVEDGATMTFFKKSKISTYDKMWAFMSSRRQSVLVKSNEEGIQRVLT

TM4

SKGTYAFLMETTNLQYYVQRNCELTQIGESFGEKHYGIAVPLNADFRSNLSVGILRLSERGELFKLRNKWFNSNESTCDSNVPTIDDGQFDMDSVGGLFVVLIVGVV SD - - YAFLMESTTIEFVTQRNCNLTQIGGLIDSKGYGVGTPMGSPYRDKITIAILQLQEEGKLHMMKEKWWRGNGCPEEE - - SKEASALGVQNIGGIFIVLAAGLV

VGLVIGVAEFLWHVQRISVKEKIPPMLALKAEFYFVIRFWLTRKPLHTYRQSRDSTSTGYSSLEQITSASSAKKKKKTRRIEK

LSVFVAVGEFLYKSKKNAQLEKESSIWLVPPYHPDTV - . 
(Figure 1-5b cont.)

GluR-IIC

GluR-6

MKKELSGNSSARWPQMFGGNSREAGMWQRILLLGCMWSAFFMCRSRGQQIN - IGAFFYDDELELEKEFMTVVNAINGPESEQTMRFYPLIKRLKPEDGSVTMQEHA - - - - - - - - MKI IFPILSNPVFRRTVKLLLCLLWIGYSQGTTHVLRFGGIFEYVESGPMGAEELAFRFAVNTINRNRTLLPNTTLTYDTQKINLYDSFEASKKA

CDLIDNGVAAIFGPSSKAASDIVALVCNSTGIPHIEFDISDEGIQAEKPNHQMTLNLYPAQAILSKAYADIVQNFGWRKFTIVYDADDARAAARLQDLLQLREVHND CDQLSLGVAAIFGPSHSSSANAVQSICNALGVPHIQTRWKH - - QVSDNKDSFYVSLYPDFSSLSRAILDLVQFFKWKTVTVVYDDSTG - - LIRLQELIKAPSRYNL

VVRVRKFHKD - DDFRVMWKSIRGER - - RVVLDCEPNMLVELLNSSTEFGLTGQYNHIFLTNLETYTDHLEELAADNETFAVNITAARLLVNPDPPPYSLPYGYVTQR RLKIRQLPADTKDAKPLLKEMKRGKEFHVIFDCSHEMAAGILKQALAMGMMTEYYHYIFTTLDLFALDVEPYRYS - - - GVNMTGFRILNTENTQVSSIIEKWSMER

DNIVYESSDPPRTLIHDLIHDALQLFAQSWRNASFFYPDRMVVPRITCDFAASGGRTWAMGRYLARLMKGTSGVNNTNFRTSILQFDEDGQRITFNIEVYDPLDG - - - - LQAPPKPDSGLLDGFMTTDAALMYDAVHVVSVAVQQFPQMT - - VSSLQCNRHKPWRFG - - TRFMSLIKEAHWEGLTGRITFNKTNGLRTDFDLDVISLKEEGL

IGIAIWDPRGQITOLNVDVKAOKKMIYRVATR - - - - - - IGPPYFSYNETARELNLTGNALYOGYAVDLIDAIARHVGFEYVFVPVADQOYGKLDKETKOWNGI IGE EKIGTWDPASGLNMTESQKGKPANITDSLSNRSLIVTTILEEPYVLFKKSD - KPLYGNDRFEGYCIDLLRELSTILGFTYEIRLVEDGKYGAQDDANGQWNGMVRE

IINNDAHMGICDLTITQARKTAVDFTVPFMQLGVSILAYKSPHVEKTLDAYLAPFGGEVWIWILISVFVMTFLKTIVARISKMDWENPHPCNRDPEVLENQWRIHNT LIDHKADLAVAPLAITYVREKVIDFSKPFMTLGISILYRKPNGTNPGVFSFLNPLSPDIWMYILLAYLGVSCVLFVIARFSPYEWYNPHPCNPDSDVVENNFTLLNS

TM2 TM3

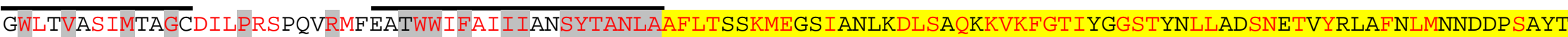
FWFGVGALMQQGSELMPKALSTRIVGGIWWFFTLIIISSYTANLAAFLTVERMESPIDSADDLAKQTKIEYGAVEDGATMTFFKKSKISTYDKMWAFMSSRRQSVLV

KDNLEGVDRVRKNRGDYMFLMETTTLEYHREQNCDLRSVGEKFGEKHYAIAVPFGAEYRSNLSVAILKLSERGELYDLKQKWWKNPNASCFEEPDPDATPDMTFEEL KSNEEGIQRVLTS - - DYAFLMESTTIEFVTQRNCNLTQIGGLIDSKGYGVGTPMGSPYRDKITIAILQLQEEGKLHMMKEKWWR - - - GNGCPEEESKEASALGVQNI

TM4

RGIFYTLYAGILIAFLIGITEFLVYVQQVALEERLTFKDAFKKEIRFVLCVWNNRKPIVAGTPISSVRTTPRRSLDKSLDRTPKSSRRVVIGRSSEEMREMAQGSGS GGIFIVLAAGLVLSVFVAVGEFLYKSKKNAQLEKE - . _ . $\ldots \ldots$

SSGSNNAGRGEKEARV

- - - - - - - - - - - - 
(Figure 1-5b cont.)

GluR-IID - -MHFCWISLIILSLSRVQAQFYGGNAYEASSGQSIRLG - - - LITDDATDRIRQTFEHAISVVNN - - - ELGVPLVGETEQVAYGNSVQAFAQLCRLMQSGVGAVF GluR-6 MKIIFPILSNPVFRRTVKLLLCLLWIGYSQGTTHVLRFGGIFEYVESGPMGAEELAFRFAVNTINRNRTLLPNTTLTYDTQKINLYDSFEASKKACDQLSLGVAAIF

GPAARHTASHLLNACDSKDIPFIYPHLSWGSNPDG - - - FNLHPSPEDIANALYDIVNQFEWSRFIFCYESAEYLKILDHLMTRYGIKGPVIKVMRYDLNLNGNYKS GPSHSSSANAVQSICNALGVPHIQTRWKHQVSDNKDSFYVSLYPDFSSLSRAILDLVQFFKWKTVTVVYDDSTGLIRLQELIKAPSRYNLRLKIRQLPADTK-DAKP

VLRRIRKSEDSRIVVVGSTTGVAELLRQAQQVGIMNEDYTYIIGNLNLHTFDLEEYKYSEANITGIRMFSPDQEEVRDLMEKLHQELGESEPVNSG - - STFITMEM LLKEMKRGKEFHVIFDCSHEMAAGILKQALAMGMMTEYYHYIFTTLDLFALDVEPYRYSGVNMTGFRILNTENTQVSSI IEKWSMERLQAPPKPDSGLLDGFMTTDA

ALTYDAVRVIAETTKHLPYQPQMLNCSERHDNVQPDGSTFRNYMRSLEIKEKTITGRIYFEGN - -VRKGFTFDVIELQTSGLVKVGTWEEGKDFEFQRPPQAVNFND ALMYDAVHVVSVAVQQFP - - - QMTVSSLQCNRHKPWRFGTRFMSLIKEAHWEGLTGRITFNKTNGLRTDFDLDVISLKEEGLEKIGTWDPASGLNMTESQKGKPANI

IDDGSLVNKTFIVLISVATKPYASLVESIDTLIGNNOFOGYGVDLIKELADKLGFNFTFRDGGN - DYGSFNKTTNSTSGMLKEIVEGRADLAITDLTITSEREEVID TDS - - - LSNRSLIVTTILEEPYVLFKKSDKPLYGNDRFEGYCIDLLRELSTILGFTYEIRLVEDGKYGAQDDANGQWNGMVRELIDHKADLAVAPLAITYVREKVID

TM1

TM2

FSIPFMNLGIAILYVKPQKAPPALFSFMDPFSSEVWLYLGIAYLGVSLCFFIIG $R L S P I E W D N P Y P C I E E P E E L E N Q \overline{F T I N N S L W F T T G A L L Q Q G S E I A P K A L S T R T ~}$ FSKPFMTLGISILYRKPNGTNPGVFSFLNPLSPDIWMYILLAYLGVSCVLFVIARFSPYEWYNPHPCNPDSDVVENNFTLLNSFWFGVGALMQQGSELMPKALSTRI

TM3

ISAIWWFFTLIMVSSYTANLAAFLTIENPTSPINSVKDLADNKDDVQYGAKRTGSTRNFFSTSEEPIYIKMNEYLN -AHPEMLMENNQOGVDKVKSGTKYAFLMEST VGGIWWFFTLIIISSYTANLAAFLTVERMESPIDSADDLAKQT-KIEYGAVEDGATMTFFKKSKISTYDKMWAFMSSRRQSVLVKSNEEGIQRVLT - SDYAFLMEST

TM4

SIEFNTVRECNLTKVGDPLDEKGYGIAMVKNWPYRDKFNKALLELQEQGVLARLKNKWWNEVGAGVCSAKSDDDGPSELGVDNLSGIYVVLVIGSIISIIISILCWC TIEFVTQRNCNLTQIGGLIDSKGYGVGTPMGSPYRDKITIAILQLQEEGKLHMMKEKWWRGNGCPEEESKE - - - - ASALGVQNIGGIFIVLAAGLVLSVFVAVGEFL

YFVYKKAKNYEVPFCDALAEEFRIVIRFSENERPLKSAQSIYSRSRNSSQSIESLKTDSEENMPVED

YKSKKNAQ - . . . . . . . . . . . . . 
(Figure 1-5b cont.)

GluR-IIE - - - - - MFFNHFVILWSLFSIHISVNWAQYENFGGYDNYQSLESVPIGLLTDQNTEQMNIVFDHAIDVANQEVGTSLTSLKEEVNYGDAYQSYGKLCRMLET

GIAGVFGPSSRHTAVHLMSICDAMDIPHIYSYMSENAEG - - - - - FNLHPHPADLAKALYSLITEFNWTRFIFLYESAEYLNILNELTTMLGKSGTVITVLRYDMQL GVAAIFGPSHSSSANAVQSICNALGVPHIQTRWKHQVSDNKDSFYVSLYPDFSSLSRAILDLVQFFKWKTVTVVYDDSTGLIRLQELIKAPSRYNLRLKIRQLPADT

NGNYKQVLRRVRKSVDNRIVVVGSSETMPEFLNQAQQVGIINEDYKYIIGNLDFHSFDLEEYKYSEANITGLRLFSPEKMAVKELLMKLGYPTDQDEFRNGS - - - C K-DAKPLLKEMKRGKEFHVIFDCSHEMAAGILKQALAMGMMTEYYHYIFTTLDLFALDVEPYRYSGVNMTGFRILNTENTQVSSI IEKWSMERLQAPPKPDSGLLDG

PITVEMALTYDAVQLFAQTLKNLPFKPMPQNCSQRTESVRDDGSSFKNYMRTLRLTDRLLTGPIYFEGN - -VRKGYHLDVIELQPSGIVKVGTWDEDRQYRPQRLAP FMTTDAALMYDAVHVVSVAVQQFPQMTVSSLQCNRHKPWR - FGTRFMSLIKEAHWEG - - LTGRITFNKTNGLRTDFDLDVISLKEEGLEKIGTWDPASGLN - MTES

TTAOFDSVDNSLANKTFIILLSVPNKPYAOLVETYKOLEGNSOYEGYGVDLIKELADKLGFNFTFVNGGN - DYGSYNKSTNESTGMLREIMTGRADLAITDLTITSE QKGKPANITDSLSNRSLIVTT - ILEEPYVLFKKSDKPLYGNDRFEGYCIDLLRELSTILGFTYEIRLVEDGKYGAQDDANGQWNGMVRELIDHKADLAVAPLAITYV

TM2

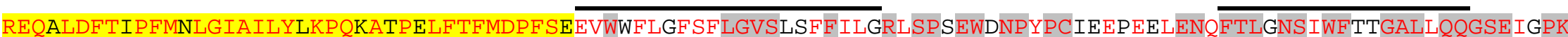
REKVIDFSKPFMTLGISILYRKPNGTNPGVFSFLNPLSPDIWMYILLAYLGVSCVLFVIARFSPYEWYNPHPCNPDSDVVENNFTLLNSFWFGVGALMQQGSELMPK

TM3

ALSTRTVASFWWFFTLIVVSSYTANLAAFLTIEKPOSLINSVDDLADNKDGVVYGAKKTGSTRNFFMTSAEERYKKMNKFMSENPO -YLTEDNMEGVNRVKTNTHYA ALSTRIVGGIWWFFTLIIISSYTANLAAFLTVERMESPIDSADDLAKQTK-IEYGAVEDGATMTFFKKSKISTYDKMWAFMSSRRQSVLVKSNEEGIQRVLT -SDYA

TM4

FLMESTSIEYNTKRECNLKKIGDALDEKGYGIAMRKDWPHRGKFNNALLELQEQGVLEKMKNKWWNEVGTGICATKEDAPDATPLDMNNLE $\overline{G V F F V L L V G S C C A L L Y ~}$ FLMESTTIEFVTQRNCNLTQIGGLIDSKGYGVGTPMGSPYRDKITIAILQLQEEGKLHMMKEKWWRGNG - - - - CPEEESKEASALGVQNIGGIFIVLAAG - - - - - - -

GIISWVLFVMKKAHHYRVPLRDALKEEFQFVIDFNNYVRVLKNSASIYSRSRQSSMSVASVAQESQ

- - LVLSVFVAVGEFLYKSKKNAQLEKESSIWLVPPYHPDTV - . . . . . . . . . . . . . . 
(Figure 1-5b cont.)

GluR-IIA

GluR-7

- - - -MRLCPVVIYAFIIIIGFLEGIIALGGDDRNEITVGAIFYENE - - - - - - - KEIELSFDQAFREVNNMKFSELRFVTIKRYMPTNDSFLLQQITCELISNGVA MTAPWRRLRSLVWEYWAGLLVCAFWIPDSRGMPHVIRIGGIFEYADGPNAQVMNAEEHAFRFSANI INRNRTLLPNTTLTYDIQRIHFHDSFEATKKACDQLALGVV

AIFGPSSKAASDIVAQIANATGIPHIEYDLKLEATRQEQLNHQMSINVAPSLSVLSRAYFEIIKSNYEWRTFTLIYETPEGLARLQDLMNIQALNSDYVKLRNLADY AIFGPSQGSCTNAVQSICNALEVPHIQLRWKHHPLDNK - - DTFYVNLYPDYASLSHAILDLVQY - LKWRSATVVYDDSTGLIRLQELIMAPSRYNIRLKIRQLPID

ADDYRILWKETDETFHEQRI ILDCEPKTLKELLKVSIDFKLQGPFRNWFLTHLDTHNSGLRDIYNEDFKAN - - ITSVRLKVVDANPFERKKTRLTKVDQILGNQTM SDDSRPLLKEMKR - GREFRI IFDCSHTMAAQILKQAMAMGMMTEYYHFIFTTLDLYALDLEPYRYSGVNLTGFRILNVDNPHVSAIVEKWSMERLQAAPRSESGLLD

LPILIYDAVVLFASSARNVIAAMQPFHPPN - RHCGSSSPWMLGAFIVNEMKTISEDDVEPHFKTENMKLDEYGQRIHFNLEIYKPTVN - -EPMMVWTPDNGIKKRLL GVMMTDAALLYDAVHIVSVCYQRAPQMTVNSLQCHRHKAWRFGGRFMNFIKEAQWEGLTGRIVFN - - - KTSGLRTDFDLDIISLKEDGLEKVGVWSPADGLN - - - I

NLELESAGTTODFSEORKVYTVVTHYEEPYFMMKEDHENFRGREKYEGYAVDLISKLSELMEFDYEFMIVNGNGKYNP - -ETKOWDGIIRKLIDHHAOIGVCDLTIT TEVAKGRGPNVTDSLTNRSLIVTTVLEEPFVMFRKSDRTLYGNDRFEGYCIDLLKELAHILGFSYEIRLVE-DGKYGAQDDKGQWNGMVKELIDHKADLAVAPLTIT

TM2

QMRRSVVDFTVPFMQLGISILHYKSPPEPKNQFAFLEPFAVEVWIYMIFAQLIMTLAFVFIARLSYREWLPPNPAIQDPDELENIWNVNNSTWLMVGSIMQQGCDIL HVREKAIDFSKPFMTLGVSILYRKPNGTNPSVFSFLNPLSPDIWMYVLLAYLGVSCVLFVIARFSPYEWYDAHPCNPGSEVVENNFTLLNSFWFGMGSLMQQGSELM

\section{TM3}

PRGPHMRILTGMWWFFALMMLSTYTANLAAFLTSNKWOSSIKSLQDLIEODKVHFGSMRGGSTSLFFSESNDTDYQRAWNOMKDFNPSAFTSTNKEGVARVRKEKGG PKALSTRIIGGIWWFFTLIIISSYTANLAAFLTVERMESPIDSADDLAKQTKIEYGAVKDGATMTFFKKSKISTFEKMWAFMSSK - PSALVKNNEEGIQRALT - -AD

TM4

YAFLMETTSLTYNIERNCDLTQIGEQIGEKHYGLAVPLGSDYRTNLSVSILQLSERGELQKMKNKWWKNHNVTCDSYHEVDGDELSIIELGGVFLVLAGGVLIGVIL YALLMESTTIEYVTQRNCNLTQIGGLIDSKGYGIGTPMGSPYRDKITIAILQLQEEDKLHIMKEKWWRGS - - GCPEEENKEASALGIQKIGGIFIVLAAGLVLSVLV

GIFEFLWNVQNVAVEERVTPWQAFKAELIFALKFWVRKKPMRISSSSDKSSSRRSSGSRRSSKEKSRSKTVS

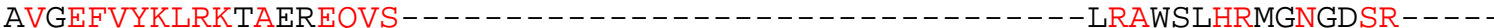


(Figure 1-5b cont.)

GluR-IIB

- - - - - - MHGLQFLVLLALAIASGANEDT - - - LVIKIGAIFFDTEMKLADAFSAALEEVNAIN - - - - - - - - PALKLDAIKRYVTVDDSIVLQDISCDLIGSGVA MTAPWRRLRSLVWEYWAGLLVCAFWIPDSRGMPHVIRIGGIFEYADGPNAQVMNAEEHAFRFSANIINRNRTLLPNTTLTYDIQRIHFHDSFEATKKACDQLALGVV

AIFGPSSKTNSDIVEVLCNMTGIPHLQFDWHPQQSNRERMNHQLTVNVAPMELFLSAAFSDILASKTFDWKSFTIAYERSSHLIRLQHILAWKQLHKAGIKMQEFER AIFGPSQGSCTNAVQSICNALEVPHIQLRWKHHPLDNK - - DTFYVNLYPDYASLSHAILDLVQ - -YLKWRSATVVYDDSTGLIRLQELIMAPSRYNIRLKIRQLPI

- GDDYRILWKRINNAREKFVLLDCPSDILVDVINASIGYNMTGSFNHLFLTNLDTHLSGIDGFYSRDFTVAVAAVRIRTYVPPPVHDEIDVFDNS - - - VDTRFSSL DSDDSRPLLKEMKRGREFRI IFDCSHTMAAQILKQAMAMGMMTEYYHFIFTTLDLYALDLEPYRYSG - -VNLTGFRILNVDNPHVSAIVEKWSMERLQAAPRSESGL

GSQLVYDSIVLFYNALLEIS - - - QRPGFYIPNFSCGRG - FWQPGPRLVEQMKQITPKMVKPPFKTQRLQINADGQREDFNLEVYNPIIDRVTHIWNKEFQLVDFEK LDGVMMTDAALLYDAVHIVSVCYQRAPQMTVNSLQCHRHKAWRFGGRFMNFIKEAQWEGLTGRIVFN - - - -KTSGLRTDFDLDIISLKEDGLEKVG - - - - - VWSPA

LRENSTOALKOKRLONKEDFSOKPIRYTVATRVGKPYFSWREEPEGVHYEGNERFEGYAVDLIYMLAOECKFDFNFEPVRDNKYGSYDANTDEWDGI IROLIDNNAO DGLNITEVAKGRGPNVTDSLTNR - -SLIVTTVLEEPFVMFRKSDR - - TLYGNDRFEGYCIDLLKELAHILGFSYEIRLVEDGKYGAQDD - KGQWNGMVKELIDHKAD

IGICDLTITQARRSVVDFTVPFMQLGISILSYKEPPPKADIYAFLNPYNAEVWLFVMIAMMITAFALIFTGRIDQYEWDQPVENVNREMERONIWHLSNALWLVLGS LAVAPLTITHVREKAIDFSKPFMTLGVSILYRKPNGTNPSVFSFLNPLSPDIWMYVLLAYLGVSCVLFVIARFSPYEWYDAHPCNPGSEVVENNFTLLNSFWFGMGS

\section{TM3}

$\overline{M L N Q G C D L L P R G L P M R L L T A F W W I F A L L I S Q T Y I A K L A A F I T S S K I A G D I G S L H D L V D Q N K V Q F G T I R G G A T S V Y F S E S N D T D N R M A W N K M L S F K P D A F T K N N E E G V ~}$ LMQQGSELMPKALSTRIIGGIWWFFTLIIISSYTANLAAFLTVERMESPIDSADDLAKQTKIEYGAVKDGATMTFFKKSKISTFEKMW-AFMSSKPSALVKNNEEGI

DRVKLSKGTYAFLMETTNLQYYVQRNCELTQIGESFGEKHYGIAVPLNADFRSNLSVGILRLSERGELFKLRNKWFNSNESTCDSNVPTIDDGQFDMDSVGGLFVVL QRALTAD - - YALLMESTTIEYVTQRNCNLTQIGGLIDSKGYGIGTPMGSPYRDKITIAILQLQEEDKLHIMKEKWWRGSGCPEEENK - - EASALGIQKIGGIFIVL

TM4

IVGVVVGLVIGVAEFLWHVQRISVKEKIPPMLALKAEFYFVIRFWLTRKPLHTYRQSRDSTSTGYSSLEQITSASSAKKKKKTRRIEK

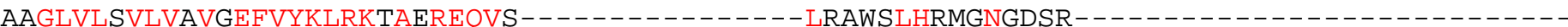


(Figure 1-5b cont.)

GluR-IIC MKKELSGNSSARWPQMFGGNSREAGMWQRILLLGCMWSAFFMCRSRGQQINIGAFFYDDELELEKEFMTVVNAINGPESEQTMRFYPLIKRLKPEDGSVTMQEHACD GluR-7 MTAPWRRLRSLVWEYWAG - - - - - - LLVCAFWIPDSRGMPHVIRIGGIFEYADGPNAQVMNAEEHAFRFSANI INRNRTLLPNTTLTYDIQRIHFHDSFEATKKACD

LIDNGVAAIFGPSSKAASDIVALVCNSTGIPHIEFDISDEGIQAEKPNHQMTLNLYPAQAILSKAYADIVQNFGWRKFTIVYDADDARAAARLQDLLQLREVHNDVV QLALGVVAIFGPSQGSCTNAVQSICNALEVPHIQLRWKHHPLDNK - - DTFYVNLYPDYASLSHAILDLVQYLKWRSATVVYDDSTG - - LIRLQELIMAPSRYNIRL

RVRKFHKD - DDFRVMWKSIRGER - -RVVLDCEPNMLVELLNSSTEFGLTGQYNHIFLTNLETYTDHLEELAADNETFAVNITAARLLVNPDPPPYSLPYGYVTQRDN KIRQLPIDSDDSRPLLKEMKRGREFRIIFDCSHTMAAQILKQAMAMGMMTEYYHFIFTTLDLYALDLEPYRYS - - - GVNLTGFRILNVDNPHVSAIVEKWSMER - -

IVYESSDPPRTLIHDLIHDALQLFAQSWRNASFFYPDRMVVPRITCDFAASGGRTWAMGRYLARLMKGTSGVNNTNFRTSILQFDEDGQRITFNIEVYDPLDG - IG - LQAAPRSESGLLDGVMMTDAALLYDAVHIVSVCYQR - -APQMTVNSLQCHRHKAWRFG - - GRFMNFIKEAQWEGLTGRIVFNKTSGLRTDFDLDI ISLKEDGLEK

IAIWDPRGQITQLNVDVKAQKKMIYRVATR - - - - - - IGPPYFSYNETARELNLTGNALYQGYAVDLIDAIARHVGFEYVFVPVADQQYGKLDKETKQWNGI IGEII VGVWSPADGLNITEVAKGRGPNVTDSLTNRSLIVTTVLEEPFVMFRKSDRTL - - YGNDRFEGYCIDLLKELAHILGFSYEIRLVEDGKYGAQD - DKGQWNGMVKELI

TM1

NNDAHMGICDLTITOARKTAVDFTVPFMQLGVSILAYKSPHVEKTLDAYLAPFGGEVWIWILISVFVMTFLKTIVARISKMDWENPHPCNRDPEVLENQWR $\overline{\text { IHNTGW }}$ DHKADLAVAPLTITHVREKAIDFSKPFMTLGVSILYRKPNGTNPSVFSFLNPLSPDIWMYVLLAYLGVSCVLFVIARFSPYEWYDAHPCNPGSEVVENNFTLLNSFW

TM2 TM3

LTVASIMTAGCDILPRSPQVRMFEATWWIFAIIIANSYTANLAAFLTSSKMEGSIANLKDLSAQKKVKFGTIYGGSTYNLLADSNETVYRLAFNLMNNDDPSAYTKD FGMGSLMQQGSELMPKALSTRIIGGIWWFFTLIIISSYTANLAAFLTVERMESPIDSADDLAKQTKIEYGAVKDGATMTFFKKSKISTFEKMWAFMSSK - PSALVKN

NLEGVDRVRKNRGDYMFLMETTTLEYHREQNCDLRSVGEKFGEKHYAIAVPFGAEYRSNLSVAILKLSERGELYDLKQKWWKNPNASCFEEPDPDATPDMTFEELRG NEEGIQRALT - -ADYALLMESTTIEYVTQRNCNLTQIGGLIDSKGYGIGTPMGSPYRDKITIAILQLQEEDKLHIMKEKWWRG - -SGCPEEENKEAS - ALGIQKIGG

TM4

IFYTLYAGILIAFLIGITEFLVYVQQVALEERLTFKDAFKKEIRFVLCVWNNRKPIVAGTPISSVRTTPRRSLDKSLDRTPKSSRRVVIGRSSEEMREMAQGSGSSS

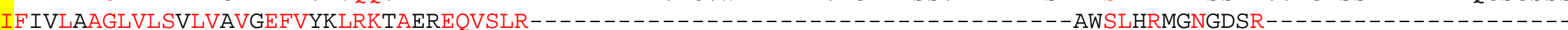

GSNNAGRGEKEARV

- . - . - - - 
(Figure 1-5b cont.)

GluR-IID MHFCWISLIILSLSRVQAQFYGGNAYEASSGQ - -SIRLG - - LITDDATDRIRQTFEHAISVVNNELG - - - - - - VPLVGETEQVAYGNSVQAFAQLCRLMQSGVG GluR-7 MTAPWRRLRSLVWEYWAGLLVCAFWIPDSRGMPHVIRIGGIFEYADGPNAQVMNAEEHAFRFSANIINRNRTLLPNTTLTYDIQRIHFHDSFEATKKACDQLALGVV

AVFGPAARHTASHLLNACDSKDIPFIYPHLSWGSNPDG - - - FNLHPSPEDIANALYDIVNQFEWSRFIFCYESAEYLKILDHLMTRYGIKGPVIKVMRYDLNLNGN AIFGPSQGSCTNAVQSICNALEVPHIQLRWKHHPLDNKDTFYVNLYPDYASLSHAILDLVQYLKWRSATVVYDDSTGLIRLQELIMAPSRYNIRLKIRQLPIDSD - D

YKSVLRRIRKSEDSRIVVVGSTTGVAELLRQAQQVGIMNEDYTYIIGNLNLHTFDLEEYKYSEANITGIRMFSPDQEEVRDLMEKLHQELGESEPVNSG - - -STFIT SRPLLKEMKRGREFRI IFDCSHTMAAQILKQAMAMGMMTEYYHFIFTTLDLYALDLEPYRYSGVNLTGFRILNVDNPHVSAIVEKWSMERLQAAPRSESGLLDGVMM

MEMALTYDAVRVIAETTKHLPYQPQMLNCSERHDNVQPDGSTFRNYMRSLEIKEKTITGRIYFEGNVRKGFTFDVIELQTSGLVKVGTWEEGKDFEFQRPPQAVNFN TDAALLYDAVHIVSVCYQRAPQMTVNSLQCHRHKAWRFGG - RFMNFIKEAQWEGLTGRIVFNKTSGLRTDFDLDIISLKEDGLEKVGVWSPADGLNITEVAKGRGPN

DIDDGSLVNKTFIVLISVATKPYASLVESIDTLIGNNOFOGYGVDLIKELADKLGFNFTFRDGGNDYGSFNKTTNSTSGMLKEIVEGRADLAITDLTITSEREEVID VTDS - - - LTNRSLIVTTVLEEPFVMFRKSDRTLYGNDRFEGYCIDLLKELAHILGFSYEIRLVEDGKYGAQDDKGQWNGMVKELIDHKADLAVAPLTITHVREKAID

TM2

FSIPFMNLGIAILYVKPQKAPPALFSFMDPFSSEVWLYLGIAYLGVSLCFFIIGRLSPIEWDNPYPCIEEPEELENQFTINNSLWFTTGALLQQGSEIAPKALSTRT FSKPFMTLGVSILYRKPNGTNPSVFSFLNPLSPDIWMYVLLAYLGVSCVLFVIARFSPYEWYDAHPCNPGSEVVENNFTLLNSFWFGMGSLMQQGSELMPKALSTRI

TM3

ISAIWWFFTLIMVSSYTANLAAFLTIENPTSPINSVKDLADNKDDVOYGAKRTGSTRNFFSTSEEPIYIKMNEYLNAHPEMLMENNOOGVDKVKSGTKYAFLMESTS IGGIWWFFTLIIISSYTANLAAFLTVERMESPIDSADDLAKQT - KIEYGAVKDGATMTFFKKSKISTFEKMWAFMSSKPSALVKNNEEGIQRALT - ADYALLMESTT

TM4

IEFNTVRECNLTKVGDPLDEKGYGIAMVKNWPYRDKFNKALLELQEQGVLARLKNKWWNEVGAGVCSAKSDDDGPSELGVDNLSGIYVVLVIGSIISIIISILCWCY IEYVTQRNCNLTQIGGLIDSKGYGIGTPMGSPYRDKITIAILQLQEEDKLHIMKEKWWRGSGC - - - PEEENKEASALGIQKIGGIFIVLAAGLVLSVLVAVGEFVY

FVYKKAKNYEVPFCDALAEEFRIVIRFSENERPLKSAQSIYSRSRNSSQSIESLKTDSEENMPVED

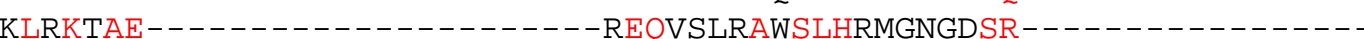


(Figure 1-5b cont.)

GluR-IIE - - MFFNHFVILWSLFSIHISVNWAQYENFGGYDNYQS - - LESVPIGLLTDQNTEQMNIVFDHAIDVANQEVG - -TSLTSLKEEVNYGDAYQSYGKLCRMLETGIA GluR-7 MTAPWRRLRSLVWEYWAGLLVCAFWIPDSRGMPHVIRIGGIFEYADGPNAQVMNAEEHAFRFSANI INRNRTLLPNTTLTYDIQRIHFHDSFEATKKACDQLALGVV

GVFGPSSRHTAVHLMSICDAMDIPHIYSYMSENAEG - - - - - FNLHPHPADLAKALYSLITEFNWTRFIFLYESAEYLNILNELTTMLGKSGTVITVLRYDMQLNGN AIFGPSQGSCTNAVQSICNALEVPHIQLRWKHHPLDNKDTFYVNLYPDYASLSHAILDLVQYLKWRSATVVYDDSTGLIRLQELIMAPSRYNIRLKIRQLPIDSD - D

YKQVLRRVRKSVDNRIVVVGSSETMPEFLNQAQQVGIINEDYKYIIGNLDFHSFDLEEYKYSEANITGLRLFSPEKMAVKELLMKLGYPTDQDEFRNGS - - - CPIT SRPLLKEMKRGREFRI IFDCSHTMAAQILKQAMAMGMMTEYYHFIFTTLDLYALDLEPYRYSGVNLTGFRILNVDNPHVSAIVEKWSMERLQAAPRSESGLLDGVMM

VEMALTYDAVQLFAQTLKNLPFKPMPQNCSQRTESVRDDGSSFKNYMRTLRLTDRLLTGPIYFEGN - -VRKGYHLDVIELQPSGIVKVGTWDEDRQYRPQRLAPTTA TDAALLYDAVHIVSVCYORAPQMTVNSLQCHRHKAWRFGG - RFMNFIKEAOWEG - - LTGRIVFNKTSGLRTDFDLDIISLKEDGLEKVGVWSPADGLNITEVAK - - $G$

OFDSVDNSLANKTFIILLSVPNKPYAOLVETYKOLEGNSOYEGYGVDLIKELADKLGFNFTFVNGGNDYGSYNKSTNESTGMLREIMTGRADLAITDLTITSEREOA RGPNVTDSLTNRSLIVTT - VLEEPFVMFRKSDRTLYGNDRFEGYCIDLLKELAHILGFSYEIRLVEDGKYGAQDDKGQWNGMVKELIDHKADLAVAPLTITHVREKA

\section{TM2}

LDFTIPFMNLGIAILYLKPQKATPELFTFMDPFSEEVWWFLGFSFLGVSLSFFILGRLSPSEWDNPYPCIEEPEELENQFTLGNSIWFTTGALLQQGSEIGPKALST IDFSKPFMTLGVSILYRKPNGTNPSVFSFLNPLSPDIWMYVLLAYLGVSCVLFVIARFSPYEWYDAHPCNPGSEVVENNFTLLNSFWFGMGSLMQQGSELMPKALST

TM3

RTVASFWWFFTLIVVSSYTANLAAFLTIEKPOSLINSVDDLADNKDGVVYGAKKTGSTRNFFMTSAEERYKKMNKFMSENPOYLTEDNMEGVNRVKTNTHYAFLMES RI IGGIWWFFTLIIISSYTANLAAFLTVERMESPIDSADDLAKQTK - IEYGAVKDGATMTFFKKSKISTFEKMWAFMSSKPSALVKNNEEGIQRALT-ADYALLMES

TM4

TSIEYNTKRECNLKKIGDALDEKGYGIAMRKDWPHRGKFNNALLELQEQGVLEKMKNKWWNEVGTGICATKEDAPDATPLDMNNLEGVFFVLLVGSCCALLYGIISW TTIEYVTQRNCNLTQIGGLIDSKGYGIGTPMGSPYRDKITIAILQLQEEDKLHIMKEKWWRGSG - - - - CPEEENKEASALGIQKIGGIFIVLAAGLVLSVLVAVGEF

VLFVMKKAHHYRVPLRDALKEEFQFVIDFNNYVRVLKNSASIYSRSRQSSMSVASVAQESQ

VYKLRKTAEREQVSLR - . . . . . . . . . . - AWSLHRMGNGDSR - . . . . . . . . . . 
(Figure 1-5b cont.)

GluR-IIA MRLCPVVIYAFIIIIGFLEGIIALGGDDRNEITVGAIFYENEKE-IELSFDQAFREVNNMKFSELRFVTIKRYMPTNDSFLLQQITCELISNGVAAIFGPSSK-AAS

DIVAQIANATGIPHIEYDLKLEATRQEQLNHQMSINVAPSLSVLSRAYFEIIKSNYEWRTFTLIYETPEGLARLQDLMNIQALNSDYVKLRNLADYADDYRILWKET SIISNICGEKEVPHFKVAP - -EEFVKFQFQRFTTLNLHPSNTDISVAVAGILN - FFNCTTACLICAKAECLLNLEKLLRQFLISKDTLSVRMLDDTRDPTPLLKEIR

DETFHEQRIILDCEPKTLKELLKVSIDFKLQGPFRNWFLTHLDTHNSGLRDIYNEDFKAN - - ITSVRLKVVDANPFERKKTRLTKVDQILGNQTMLPILIYDAVVL DD - - KTATIIIHANASMSHTILLKAAELGMVSAYYTYIFTNLEFSLQRMDSLVDDRVNILGFSIFNQSHAFFQEFAQSLNQSWQENCDHVPFTGPALSSALLFDAVY

FASSARNVIAAMQPFHPPNRHCGSSSPWMLGAFIVNEMKTISEDDVEPHFKTENMKLDEYGQRIHFNLEIYKPTVN - EPMMVWTPDNGIKKRLLNLELESAGTTQD AVVTAVQELNRSQEIGVKPLSCGSAQIWQHGTSLMNYLRMVELEGLTGHIEFN - - - - SKGQRSNYALKILQFTRNGFRQIGQWHVAEGLS - - MDSHLYASNISDT

FSEQRKVYTVVTHYEEPYFMMKEDHENFRGREKYEGYAVDLISKLSELMEFDYEFMIVNGNGKYNPETK-QWDGI IRKLIDHHAQIGVCDLTITQMRRSVVDFTVPF LFN - - TTLVVTTILENPYLMLKGNHQEMEGNDRYEGFCVDMLKELAEILRFNYKIRLVGDGVYGVPEANGTWTGMVGELIARKADLAVAGLTITAEREKVIDFSKPF

MQLGISILHYKSPPEPKNQFAFLEPFAVEVWIYMIFAQLIMTLAFVFIARLSYREWLPPNPAIQ - DPDELENIWNVNNSTWLMVGSIMQQGCDILPRGPHMRILTGM MTLGISILYRVHMGRKPGYFSFLDPFSPGVWLFMLLAYLAVSCVLFLVARLTPYEWYSPHPCAQGRCNLLVNQYSLGNSLWFPVGGFMQQGSTIAPRALSTRCVSGV

TM3

WWFFALMMLSTYTANLAAFLTSNKWOSSIKSLODLIEQDKVHFGSMRGGSTSLFFSESNDTDYQRAWNQMKDFNPSAFTSTNKEGVARVRKEKGGYAFLMETTSLTY WWAFTLIIISSYTANLAAFLTVQRMDVPIESVDDLADQTAIEYGTIHGGSSMTFFQNSRYQTYQRMWNYMYSKQPSVFVKSTEEGIARVLNSN - -YAFLLESTMNEY

\section{TM4}

NIERNCDLTQIGEQIGEKHYGLAVPLGSDYRTNLSVSILQLSERGELQKMKNKWWKNHNVTCDSYHEVDGDELSIIELGGVFLVLAGGVLIGVILGIFEFLWNVQN YRQRNCNLTQIGGLLDTKGYGIGMPVGSVFRDEFDLAILQLQENNRLEILKRKWWEGGKCPKEEDHRAKG - - LGMENIGGIFVVLICGLIVAIFMAMLEFLWTLRHS

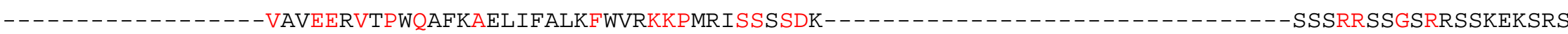
EATEVSVCQEMVTELRSIILCQDSIHPRRRRAAVPPPRPPIPEERRPRGTATLSNGKLCGAGEPDQLAQRLAQEAALVARGCTHIRVCPECRRFQGLRARPSPARSE KTVS - - - . - . - -

ESLEWEKTTNSSEPE 
(Figure 1-5b cont.)

GluR-IIB MHGLQFLVLLALAIASGANEDTLVIKIGAIFFD-TEMKLADAFSAALEEVNAINPALKLDAIKRYVTVDDSIVLQDIS - - - - CDLIGSGVAAIFGPSSK -TNSDIV KA1 MPRVSAPLVLLPAWLVMVACSPHSLRIAAILDDPMECSRGERLSITLAKNRINRAPERLGKAKVEVDIFELLRDSEYETAETMCQILPKGVVAVLGPSSSPASSSII

EVLCNMTGIPHLQFDWHPQQSNRERMNHQLTVNVAPMELFLSAAFSDILASKTFDWKSFTIAYERSSHLIRLQHILAWKQLHKAGIKMQEFERGDDYRILWKRINNA SNICGEKEVP - - HFKVAPEEFVKFQFQRFTTLNLHPSNTDISVAVAGILN - - FFNCTTACLICAKAECLLNLEKLLRQFLISKDTLSVRMLDDTRDPTPLLKEIRDD

REKFVLLDCPSDILVDVINASIGYNMTGSFNHLFLTNLDTHLSGIDGFYSR - - - - DFTVAVAAVRIRTYVPPPVHDEIDVFDNSVDTRFSSLGSQLVYDSIVLFYN KTATIIIHANASMSHTILLKAAELGMVSAYYTYIFTNLEFSLQRMDSLVDDRVNILGFSIFNQSHAFFQEFAQSLNQSWQENCDHVPFTGPALSSALLFDAVYAVVT

ALLEISQRPGFYIPNFSCG - RGFWQPGPRLVEQMKQITPKMVKPPFKTQRLQINADGQREDFNLEVYNPIIDRVTHIWNKEFQLVDFEKLRENSTQALKQKRLQNKE AVQELNRSQEIGVKPLSCGSAQIWQHGTSLMNYLR - - - - MVELEGLTGHIEFNSKGQRSNYALKILQFTRNGFRQIG - - QWHVAEGLSMDSHLYASNISDTLFN - -

DFSQKPIRYTVATRVGKPYFSWREEPEGVHYEGNERFEGYAVDLIYMLAQECKFDFNFEPVRDNKYGSYDANTDEWDGIIRQLIDNNAQIGICDLTITQARRSVVDF - - - - - - TTLVVTTILENPYLMLKG - - NHQEMEGNDRYEGFCVDMLKELAEILRFNYKIRLVGDGVYGVPEAN - GTWTGMVGELIARKADLAVAGLTITAEREKVIDF

TVPFMQLGISILSYKEPPPKADIYAFLNPYNAEVWLFVMIAMMITAFALIFTGRIDQYEWDQPVENVN - REMERQNIWHLSNALWLVLGSMLNQGCDLLPRGLPMRL SKPFMTLGISILYRVHMGRKPGYFSFLDPFSPGVWLFMLLAYLAVSCVLFLVARLTPYEWYSPHPCAQGRCNLLVNQYSLGNSLWFPVGGFMQQGSTIAPRALSTRC

TM3

LTAFWWIFALLISQTYIAKLAAFITSSKIAGDIGSLHDLVDQNKVQFGTIRGGATSVYFSESNDTDNRMAWNKMLSFKPDAFTKNNEEGVDRVKLSKGTYAFLMETT VSGVWWAFTLIIISSYTANLAAFLTVQRMDVPIESVDDLADQTAIEYGTIHGGSSMTFFQNSRYQTYQRMWNYMYSKQPSVFVKSTEEGIARVLNSN - - YAFLLEST

TM4

NLQYYVQRNCELTQIGESFGEKHYGIAVPLNADFRSNLSVGILRLSERGELFKLRNKWFNSNESTCDSNVPTIDDGQFDMDSVGGLFVVLIVGVVVGLVIGVAEFLW MNEYYRQRNCNLTQIGGLLDTKGYGIGMPVGSVFRDEFDLAILQLQENNRLEILKRKWWEGGKCPKEEDHRAKGLG - - MENIGGIFVVLICGLIVAIFMAMLEFLW

HVQR - - - - ISVKEKIPPMLALKAEFYFVIRFWLTRKPLHTYRQ - -SRDSTSTGYSSLEQITSASSAKKKKKTRRIEK - - - - - - - - - - - - - - - - - TLRHSEATEVSVCQEMVTELRSIILCQDSIHPRRRRAAVPPPRPPIPEERRPRGTATLSNGKLCGAGEPDQLAQRLAQEAALVARGCTHIRVCPECRRFQGLRARPS

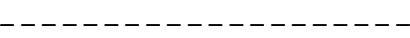

PARSEESLEWEKTTNSSEPE 
(Figure 1-5b cont.)

GluR-IIC -MKKELSGNSSARWPQMFGGNSREAGMWQRILLLGCMWSAFFMCRSRGQQINIGAFFYDDELELEKEFMTVVNAINGPESEQTMRFYPLIKRLKPEDGSVTMQEHAC KA1 MPRVSAPLVLLPAWLVMVACSP - - - - - HSLRIAAILDDPMECS - RGERLSI - - - - - - - TLAKNRINRAPERLG - KAKVEVDIFELLR- - - - DSEYETAETMC

DLIDNGVAAIFGPSSK-AASDIVALVCNSTGIPHIEFDISDEGIQAEKPNHQMTLNLYPAQAILSKAYADIVQNFGWRKFTIVYDADDARAAARLQDLLQLREVHND QILPKGVVAVLGPSSSPASSSIISNICGEKEVP - - HFKVAPEEFVKFQFQRFTTLNLHPSNTDISVAVAGILNFFNCTTACLICAK - - AECLLNLEKLLRQFLISKD

VVRVRKFHKDDDFRVMWKSIRGER - -RVVLDCEPNMLVELLNSSTEFGLTGQYNHIFLTNLETYTDHLEELAADNETFAVNITAARLLVNPDPPPYSLPYGYVTQRD TLSVRMLDDTRDPTPLLKEIRDDKTATIIIHANASMSHTILLKAAELGMVSAYYTYIFTNLEFSLQRMDSLVDDRVNILGFSIFNQSHAFFQEFAQSLNQSWQENCD

NIVYESSDPPRTLIHDLIHDALQLFAQSWRNASFFYPDRMVVPRITCDFAASGGRTWAMGRYLARLMKGTSGVNNTNFRTSILQFDEDGQRITFNIEVYDPLDGIGI HVPFTGPALSSALLFDAVYAVVTAVQELNR - - - - - SQEIGVKPLSCGSAQIWQHGTSLMNYLR - MVELEGLTGHIEFNSKGQRSNYALKILQFTRNGFRQIGQWHV

AIWDPRGQITQLNVDVKAQKKMIYRVATRIGPPYFSYNETARELNLTGNALYQGYAVDLIDAIARHVGFEYVFVPVADQQYGKLDKETKQWNGIIGEIINNDAHMGI AEGLSMDSHLYASNISDTLFNTTLVVTTILENPYLMLKGNHQEME - - GNDRYEGFCVDMLKELAEILRFNYKIRLVGDGVYG - VPEANGTWTGMVGELIARKADLAV

CDLTITQARKTAVDFTVPFMQLGVSILAYKSPHVEKTLDAYLAPFGGEVWIWILISVFVMTFLKTIVARISKMDWENPHPCNR-DPEVLENOWRIHNTGWLTVASIM AGLTITAEREKVIDFSKPFMTLGISILYRVHMGRKPGYFSFLDPFSPGVWLFMLLAYLAVSCVLFLVARLTPYEWYSPHPCAQGRCNLLVNQYSLGNSLWFPVGGFM

TM3

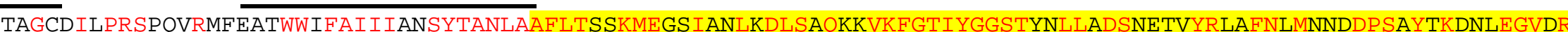
QQGSTIAPRALSTRCVSGVWWAFTLIIISSYTANLAAFLTVQRMDVPIESVDDLADQTAIEYGTIHGGSSMTFFQNSRYQTYQRMWNYMYSKQPSVFVKSTEEGIAR

VRKNRGDYMFLMETTTLEYHREQNCDLRSVGEKFGEKHYAIAVPFGAEYRSNLSVAILKLSERGELYDLKQKWWKNPNASCFEEPDPDATPDMTFEELRGIFYTLYA VLN - -SNYAFLLESTMNEYYRQRNCNLTQIGGLLDTKGYGIGMPVGSVFRDEFDLAILQLQENNRLEILKRKWWEG - -GKCPKEED - HRAKGLGMENIGGIFVVLIC

TM4

GILIAFLIGITEFLVYVQQVALEERLTFKDAFKKEIRFVLC - - - VWNNRKPIVAGTPISSVRTTPRRSLDKSLDR - - - - - - - - - - - - TPKSSRRVVIGRSSEEMRE GLIVAIFMAMLEFLWTLRHSEATEVSVCQEMVTELRSIILCQDSIHPRRRRAAVPPPRPPIPEERRPRGTATLSNGKLCGAGEPDQLAQRLAQEAALVARGCTHIRV

MAQGSGSSSGSNNAGRGEKEARV - . - . - . - . -

CPECRRFQGLRARPSPARSEESLEWEKTTNSSEPE 
(Figure 1-5b cont.)

GluR-IID MHFCWISLIILSLSRVQAQFYGGNAYEASSGQSIRLGLITDDATDRIR - - - - - QTFEHAISVVNNELG - VPLVGETEQVAYGNSVQAFAQLCRLMQSGVGAVFGPA KA1 - . - . - MPRVSAPLVLLPAWLVMVACSPHSLRIAAILDDPMECSRGERLSITLAKNRINRAPERLGKAKVEVDIFELLRDSEYETAETMCQILPKGVVAVLGPS

ARHTASHLLN - ACDSKDIPFIYPHLSWGS - - - - NPDGFNLHPSPEDIANALYDIVNQFEWSRFIFCYESAEYLKILDHLMTRYGIKGPVIKVMRYDLNLNGNYKSV SSPASSSIISNICGEKEVPHFKVAPEEFVKFQFQRFTTLNLHPSNTDISVAVAGILNFFNCTTACLICAKAECLLNLEKLLRQFLISKDTLSVRMLDDTRDP - -TPL

LRRIRKSEDSRIVVVGSTTGVAELLRQAQQVGIMNEDYTYIIGNLNLHTFDLEEYKYSEANITGIRMFSPDQEEVRDLMEKLHQELGE - - -SEPVNSGSTFITMEMA LKEIRDDKTATIIIHANASMSHTILLKAAELGMVSAYYTYIFTNLEFSLQRMDSLVDDRVNILGFSIFNQSHAFFQEFAQSLNQSWQENCDHVPFTGPALSSALLFD

LTYDAVRVIAETTKHLPYQPQMLNCSERHDNVQPDGSTFRNYMRSLEIKEKTITGRIYFEGN - VRKGFTFDVIELQTSGLVKVGTWEEGKDFEFQRPPQAVNFNDID AVYAVVTAVQELNRSQEIGVKPLSCGS - -AQIWQHGTSLMNYLRMVELEG - - LTGHIEFNSKGQRSNYALKILQFTRNGFRQIGQWHVAEGLSMDSHLYASNISDT -

DGSLVNKTFIVLISVATKPYASLVESIDTLIGNNQFQGYGVDLIKELADKLGFNFTFRDGGNDYGSFNKTTNSTSGMLKEIVEGRADLAITDLTITSEREEVIDFSI - - - - LFNTTLVVTTILENPYLMLKGNHQEMEGNDRYEGFCVDMLKELAEILRFNYKIRLVGDGVYGVPEANGTWTGMVGELIARKADLAVAGLTITAEREKVIDFSK

TM1

TM2

PFMNLGIAILYVKPQKAPPALFSFMDPFSSEVWLYLGIAYLGVSLCFFIIGRLSPIEWDNPYPCIE-EPEELENQFTINNSLWFTTGALLQQGSEIAPKALSTRTIS PFMTLGISILYRVHMGRKPGYFSFLDPFSPGVWLFMLLAYLAVSCVLFLVARLTPYEWYSPHPCAQGRCNLLVNQYSLGNSLWFPVGGFMQQGSTIAPRALSTRCVS

TM3

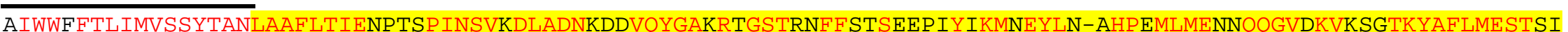
GVWWAFTLIIISSYTANLAAFLTVQRMDVPIESVDDLADQT - AIEYGTIHGGSSMTFFQNSRYQTYQRMWNYMYSKQPSVFVKSTEEGIARVLN - SNYAFLLESTMN

TM4

EFNTVRECNLTKVGDPLDEKGYGIAMVKNWPYRDKFNKALLELQEQGVLARLKNKWWNEVGAGVCSAKSDDDGPSELGVDNLSGIYVVLVIGSIISIIISILCWCYF EYYRQRNCNLTQIGGLLDTKGYGIGMPVGSVFRDEFDLAILQLQENNRLEILKRKWWEGG - - - KCPKEEDHRAKGLGMENIGGIFVVLICGLIVAIFMAMLEFLWT

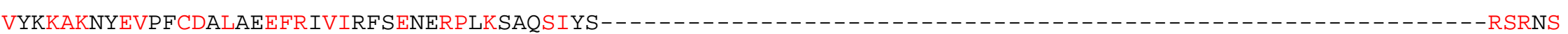
LR-HSEATEVSVCQEMVTELRSIILCQDSIHPRRRRAAVPPPRPPIPEERRPRGTATLSNGKLCGAGEPDQLAQRLAQEAALVARGCTHIRVCPECRRFQGLRARPS

-SQSIESLKTDSEENMPVED PARSEESLEWEKTTNSSEPE 
(Figure 1-5b cont.)

GluR-IIE MFFNHFVILWSLFSIHISVNWAQYENFGGYDNYQSLESVPIGLLTDQNTEQMNIVFDH-AIDVANQEVGTSLTSLKEEVNYG-DAYQSYGKLCRMLETGIAGVFGPS

SRHTAVHLMS - ICDAMDIPHIYS - - - - - - YMSENAEGFNLHPHPADLAKALYSLITEFNWTRFIFLYESAEYLNILNELTTMLGKSGTVITVLRYDMQLNGNYKQV SSPASSSI ISNICGEKEVPHFKVAPEEFVKFQFQRFTTLNLHPSNTDISVAVAGILNFFNCTTACLICAKAECLLNLEKLLRQFLISKDTLSVRMLDDTRDP - -TPL

LRRVRKSVDNRIVVVGSSETMPEFLNQAQQVGIINEDYKYIIGNLDFHSFDLEEYKYSEANITGLRLFSPEKMAVKELLMKLGYPTDQDEFRNGSCPITVEMALTYD LKEIRDDKTATI I IHANASMSHTILLKAAELGMVSAYYTYIFTNLEFSLQRMDSLVDDRVNILGFSIFNQSHAFFQEFAQSLNQSWQENCDHVPFTGPALSSALLFD

AVQLFAQTLKNLPFKPMP - - QNCSQRTESVRDDGSSFKNYMRTLRLTDRLLTGPIYFEGN - VRKGYHLDVIELQPSGIVKVGTWDEDRQYRPQRLAPTTAQFDSVDN AVYAVVTAVQELNRSQEIGVKPLSCGSAQIWQHGTSLMNYLRMVELEG - - LTGHIEFNSKGQRSNYALKILQFTRNGFRQIGQWH - - - -VAEGLSMDSHLYASNIS

SLANKTFIILLSVPNKPYAOLVETYKOLEGNSOYEGYGVDLIKELADKLGFNFTFVNGGNDYGSYNKSTNESTGMLREIMTGRADLAITDLTITSEREOALDFTIPF DTLFNTTLVVTTILENPYLMLKGNHQEMEGNDRYEGFCVDMLKELAEILRFNYKIRLVGDGVYGVPEANGTWTGMVGELIARKADLAVAGLTITAEREKVIDFSKPF

TM2

MNLGIAILYLKPQKATPELFTFMDPFSEEVWWFLGSFLGVSLSFFILGRLSPSEWDNPYPCIE-EPEELENQFTLGNSIWFTTGALLQQGSEIGPKALSTRTVASF MTLGISILYRVHMGRKPGYFSFLDPFSPGVWLFMLLAYLAVSCVLFLVARLTPYEWYSPHPCAQGRCNLLVNQYSLGNSLWFPVGGFMQQGSTIAPRALSTRCVSGV

TM3

WWFFTLIVVSSYTANLAAFLTIEKPOSLINSVDDLADNKDGVVYGAKKTGSTRNFFMTSAEERYKKMNKFMS-ENPOYLTEDNMEGVNRVKTNTHYAFLMESTSIEY WWAFTLIIISSYTANLAAFLTVQRMDVPIESVDDLADQT -AIEYGTIHGGSSMTFFQNSRYQTYQRMWNYMYSKQPSVFVKSTEEGIARVLN-SNYAFLLESTMNEY

TM4

NTKRECNLKKIGDALDEKGYGIAMRKDWPHRGKFNNALLELQEQGVLEKMKNKWWNEVGTGICATKEDAPDATPLDMNNLEGVFFVLLVGSCCALLYGIISWVLFVM YRQRNCNLTQIGGLLDTKGYGIGMPVGSVFRDEFDLAILQLQENNRLEILKRKWWEGG - - - KCPKEEDHRAKGLGMENIGGIFVVLICGLIVAIFMAML - EFLWTL

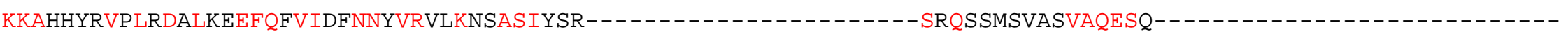
RHSEATEVSVCQEMVTELRSIILCQDSIHPRRRRAAVPPPRPPIPEERRPRGTATLSNGKLCGAGEPDQLAQRLAQEAALVARGCTHIRVCPECRRFQGLRARPSPA - - - - . - . - . - . - . -

RSEESLEWEKTTNSSEPE 
(Figure 1-5b cont.)

GluR-IIA MRLCPVVIYAFIIIIGFLEGIIALGGDDRNEITVGAIFYENEKEIELSFDQAFREVNNMKFSELRFVTIKRYMPTNDSFLLQQITCELISNGVAAIFGPSSK-AASD

IVAQIANATGIPHIEYDLKLEATRQEQLNHQMSINVAPSLSVLSRAYFEI IKSNYEWRTFTLIYETPEGLARLQDLMNIQALNSDYVKLRNLADYADDYRILWKETD TVSHICGEKEIPHIKVGP - - EETPRLQYLRFASVSLYPSNEDVSLAVSRILKS - FNYPSASLICAKAECLLRLEELVRGFLISKETLSVRMLDDSRDPTPLLKEIRD

ETFHEQRIILDCEPKTLKELLKVSIDFKLQGPFRNWFLTHLDTHNSGLRDIYNED - - - - FKANITSVRLKVVDANPFERKKTRLTKVDQILGNQTMLPILIYDAVV D - -KVSTIIIDANASISHLILRKASELGMTSAFYKYILTTMDFPILHLDGIVEDSSNILGFSMFNTSHPFYPEFVRSLNMSWRENCEASTYLG -PALSAALMFDAVH

LFASSARNVIAAMQPFHPPNRHCGSSSPWMLGAFIVNEMKTISEDDVEPHFKTENMKLDEYGQRIHFNLEIYKPTVN - -EPMMVWTPDNGIKKRLLNLELESAGTTQ VVVSAVR-ELNRSQEIGVKPLACTSANIWPHGTSLMNYLRMVEYDGLT - - - - GRVEFNSKGQRTNYTLRILEKSRQGHREIGVWYSN - - - - RTLAMNATTLDINL

DFSEQRKVYTVVTHYEEPYFMMKEDHENFRGREKYEGYAVDLISKLSELMEFDYEFMIVNGNGKYNPETK - QWDGIIRKLIDHHAQIGVCDLTITQMRRSVVDFTVP SQTLANKTLVVTTILENPYVMRRPNFQALSGNERFEGFCVDMLRELAELLRFRYRLRLVEDGLYGAPEPNGSWTGMVGELINRKADLAVAAFTITAEREKVIDFSKP

TM2

FMQLGISILHYKSPPEPKNQFAFLEPFAVEVWIYMIFAQLIMTLAFVFIARLSYREWLPPNPAIQD - PDELENIWNVNNSTWLMVGSIMQQGCDILPRGPHMRILTG FMTLGISILYRVHMGRKPGYFSFLDPFSPAVWLFMLLAYLAVSCVLFLAARLSPYEWYNPHPCLRARPHILENQYTLGNSLWFPVGGFMQQGSEIMPRALSTRCVSG

\section{TM3}

MWWFFALMMLSTYTANLAAFLTSNKWQSSIKSLQDLIEQDKVHFGSMRGGSTSLFFSESNDTDYQRAWNQMKDFNPSAFTSTNKEGVARVRKEKGGYAFLMETTSLT VWWAFTLIIISSYTANLAAFLTVQRMEVPVESADDLADQTNIEYGTIHAGSTMTFFQNSRYQTYQRMWNYMQSKQPSVFVKSTEEGIARVLNSR - - YAFLLESTMNE

\section{TM4}

YNIERNCDLTQIGEQIGEKHYGLAVPLGSDYRTNLSVSILQLSERGELQKMKNKWWKNHNVTCDSYHEVDGDELSIIELGGVFLVLAGGVLIGVILGIFEFLWNVQN YHRRLNCNLTQIGGLLDTKGYGIGMPLGSPFRDEITLAILQLQENNRLEILKRKWWEGGRCPKEEDHRAKG - - LGMENIGGIFIVLICGLIIAVFVAVMEFIWSTRR

VAVEERVTPWQAFKAELIFALKFWVRKKP - . SAESEEVSVCQEMLQELRHAVSCRKTSRSRRRRRPGGPSRALLSLRAVREMRLSNGKLYSAGAGGDAGSAHGGPQRLLDDPGPPSGARPAAPTPCTHVRVCQECRRI SSRRSSGSRRSSKEKSRSKTVS - . . . . . . . . . . . . . . . QALRASGAGAPPRGLGVPAEATSPPRPRPGPAGPRELAEHE 
(Figure 1-5b cont.)

GluR-IIB MHGLQFLVLLALAIASGANEDTLVIKIGAIFFDTEMKLAD - - AFSAALEEVN - - AINPALKLDAIKRYVTVDDSIVLQDISCDLIGSGVAAIFGPSSK - TNSDIV

EVLCNMTGIPHLQFDWHPQQSNRERMNHQLTVNVAPMELFLSAAFSDILASKTFDWKSFTIAYERSSHLIRLQHILAWKQLHKAGIKMQEFERGDDYRILWKRINNA SHICGEKEIPHIKVG - - PEETPRLQYLRFASVSLYPSNEDVSLAVSRILKS - - FNYPSASLICAKAECLLRLEELVRGFLISKETLSVRMLDDSRDPTPLLKEIRDD

REKFVLLDCPSDILVDVINASIGYNMTGSFNHLFLTNLDT - - HLSGIDGFYSRDFTVAVAAVRIRTYVPPPVHDEIDVFDNSVDTRFS - -SLGSQLVYDSIVLFYN KVSTIIIDANASISHLILRKASELGMTSAFYKYILTTMDFPILHLDGIVEDSSNILGFSMFNTSHPFYPEFVRSLNMSWRENCEASTYLGPALSAALMFDAVHVVVS

ALLEISQRPGFYIPNFSCGRG - FWQPGPRLVEQMKQITPKMVKPPFKTQRLQINADGQREDFNLEVYNPIIDRVTHIWNKEFQLVDFEKLRENSTQALKQKRLQNKE AVRELNRSQEIGVKPLACTSANIWPHGTSLMNYLR - - - - MVEYDGLTGRVEFNSKGQRTNYTLRILEKSRQGHREIG - - - - - - - - VWYSNRTLAMNATTLDINL

DFSOKPIRYTVATRVGKPYFSWREEPEGVHYEGNERFEGYAVDLIYMLAOECKFDFNFEPVRDNKYGSYDANTDEWDGIIROLIDNNAOIGICDLTITQARRSVVDF SQTLANKTLVVTTILENPYVMRR - - PNFQALSGNERFEGFCVDMLRELAELLRFRYRLRLVEDGLYGAPEPN - GSWTGMVGELINRKADLAVAAFTITAEREKVIDF

TVPFMQLGISILSYKEPPPKADIYAFLNPYNAEVWLFVMIAMMITAFALIFTGRIDQYEWDQPVENVN - REMERQNIWHLSNALWLVLGSMLNQGCDLLPRGLPMRL SKPFMTLGISILYRVHMGRKPGYFSFLDPFSPAVWLFMLLAYLAVSCVLFLAARLSPYEWYNPHPCLRARPHILENQYTLGNSLWFPVGGFMQQGSEIMPRALSTRC

TM3

LTAFWWIFALLISQTYIAKLAAFITSSKIAGDIGSLHDLVDQNKVQFGTIRGGATSVYFSESNDTDNRMAWNKMLSFKPDAFTKNNEEGVDRVKLSKGTYAFLMETT VSGVWWAFTLIIISSYTANLAAFLTVQRMEVPVESADDLADQTNIEYGTIHAGSTMTFFQNSRYQTYQRMWNYMQSKQPSVFVKSTEEGIARVLNSR - - YAFLLEST

TM4

NLQYYVQRNCELTQIGESFGEKHYGIAVPLNADFRSNLSVGILRLSERGELFKLRNKWFNSNESTCDSNVPTIDDGQFDMDSVGGLFVVLIVGVVVGLVIGVAEFLW MNEYHRRLNCNLTQIGGLLDTKGYGIGMPLGSPFRDEITLAILQLQENNRLEILKRKWWEGGRCPKEEDHRAKGLG - - MENIGGIFIVLICGLIIAVFVAVMEFIW

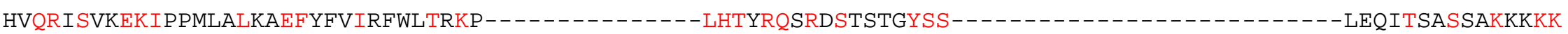
STRRSAESEEVSVCQEMLQELRHAVSCRKTSRSRRRRRPGGPSRALLSLRAVREMRLSNGKLYSAGAGGDAGSAHGGPQRLLDDPGPPSGARPAAPTPCTHVRVCQE

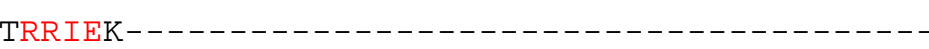

CRRIQALRASGAGAPPRGLGVPAEATSPPRPRPGPAGPRELAEHE 
(Figure 1-5b cont.)

GluR-IIC MKKELSGNSSARWPQMFGGNSREAGMWQRILLLGCMWSAFFMCRSRGQQINIGAFFYDDELELEKEFMTVVNAINGPESEQTMRFYPLIKRLKPEDGSVTMQEHACD

LIDNGVAAIFGPSSK-AASDIVALVCNSTGIPHIEFDISDEGIQAEKPNHQMTLNLYPAQAILSKAYADIVQNFGWRKFTIVYDADDARAAARLQDLLQLREVHNDV

ILPKGVVSVLGPSSSPASASTVSHICGEKEIPHIKVGP - - EETPRLQYLRFASVSLYPSNEDVSLAVSRILKSFNYPSASLICAK - - AECLLRLEELVRGFLISKET

VRVRKFHKDDDFRVMWKSIRGERR - -VVLDCEPNMLVELLNSSTEFGLTGQYNHIFLTNLETYTDHLEELAADNETFAVNITAARLLVNPDPPPYSLPYGYVTQRDN LSVRMLDDSRDPTPLLKEIRDDKVSTIIIDANASISHLILRKASELGMTSAFYKYILTTMDFPILHLDGIVED - - - - SSNILGFSMFNTSHPFYPEFVRSLNMSWRE

IVYESSDPPRTLIHDLIHDALQLFAQSWRNASFFYPDRMVVPRITCDFAASGGRTWAMGRYLARLMKGTSGVNNTNFRTSILQFDEDGQRITFNIEVYDPLDG - IG NCEASTYLGPALSAALMFDAVHVVVSAVR - - ELNRSQEIGVKPLACTSAN - - - - IWPHGTSLMNYLR - - - MVEYDGLTGRVEFNSKGQRTNYTLRILEKSRQGHRE

IAIWDPRGQITQLNVDVKAQKKMIYRVATRIGPPYFSYNETARELN - - LTGNALYQGYAVDLIDAIARHVGFEYVFVPVADQQYGKLDKETKQWNGIIGEI INNDA IGVWYSNRTLAMNATTLDINLSQTLANKTLVVTTILENPYVMRRPNFQALSGNERFEGFCVDMLRELAELLRFRYRLRLVEDGLYGAPEPN - GSWTGMVGELINRKA

HMGICDLTITQARKTAVDFTVPFMQLGVSILAYKSPHVEKTLDAYLAPFGGEVWIWILISVFVMTFLKTIVARISKMDWENPHPCNRD-PEVLENQWRIHNTGWLTV DLAVAAFTITAEREKVIDFSKPFMTLGISILYRVHMGRKPGYFSFLDPFSPAVWLFMLLAYLAVSCVLFLAARLSPYEWYNPHPCLRARPHILENQYTLGNSLWFPV

TM3

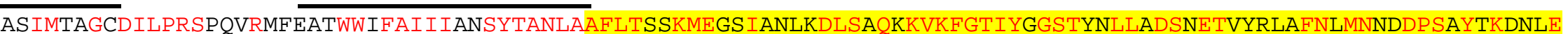
GGFMQQGSEIMPRALSTRCVSGVWWAFTLIIISSYTANLAAFLTVQRMEVPVESADDLADQTNIEYGTIHAGSTMTFFQNSRYQTYQRMWNYMQSKQPSVFVKSTEE

GVDRVRKNRGDYMFLMETTTLEYHREQNCDLRSVGEKFGEKHYAIAVPFGAEYRSNLSVAILKLSERGELYDLKQKWWKNPNASCFEEPDPDATPDMTFEELRGIFY GIARVLNSR - - YAFLLESTMNEYHRRLNCNLTQIGGLLDTKGYGIGMPLGSPFRDEITLAILQLQENNRLEILKRKWWEG - -GRCPKEED - HRAKGLGMENIGGIFI

TM4

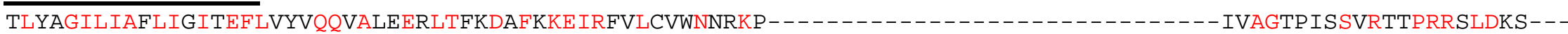
VLICGLIIAVFVAVMEFIWSTRRSAESEEVSVCQEMLQELRHAVSCRKTSRSRRRRRPGGPSRALLSLRAVREMRLSNGKLYSAGAGGDAGSAHGGPQRLLDDPGPP

LDRTPKSSRRVVIGRSSEEMREMAQGSGSSSGSNNAGRGEKEARV - . - . - . - . - . - . - .

SGARPAAPTPCTHVRVCQECRRIQALRASGAGAPPRGLGVPAEATSPPRPRPGPAGPRELAEHE 
(Figure 1-5b cont.)

GluR-IID MHFCWISLIILSLSRVQAQFYGGNAYEASSGQSIRLGLITDDATDRIRQTFEHAISVVNNELGVPLVGETEQVAYGNSVQAFAQLCRLMQSGVGAVFGPAAR-HTAS KA2 MPAELLLLLIVAFASPSCQVLSSLRMAAILDDQTVCGRGERLALALAREQINGI IEVPAKAR - - VEVDIFELQRDSQYETTDTMCQILPKGVVSVLGPSSSPASAS

HLLNACDSKDIPFIYPHLSWGS - - - - NPDGFNLHPSPEDIANALYDIVNQFEWSRFIFCYESAEYLKILDHLMTRYGIKGPVIKVMRYDLNLNGNYKSVLRRIRKS TVSHICGEKEIPHIKVGPEETPRLQYLRFASVSLYPSNEDVSLAVSRILKSFNYPSASLICAKAECLLRLEELVRGFLISKETLSVRMLDDSRDP - - TPLLKEIRDD

EDSRIVVVGSTTGVAELLRQAQQVGIMNEDYTYIIGNLNLHTFDLEEYKYSEANITGIRMFSPDQEEVRDLMEKLHQELGESEPVNSGSTFITMEMALTYDAVRVIA KVSTIIIDANASISHLILRKASELGMTSAFYKYILTTMDFPILHLDGIVEDSSNILGFSMFNTSHPFYPEFVRSLNMSWREN-CEASTYLGPALSAALMFDAVHVVV

ETTKHLPYQP - -QMLNCSERHDNVQPDGSTFRNYMRSLEIKEKTITGRIYFEGN - VRKGFTFDVIELQTSGLVKVGTWEEGKDFEFQRPPQAVNFNDIDDGSLVNKT SAVRELNRSQEIGVKPLACTSANIWPHGTSLMNYLRMVEYDG - - LTGRVEFNSKGQRTNYTLRILEKSRQG HREIGVWYSNRTLAMNATTLDINLSQT - - - - LANK

FIVLISVATKPYASLVESIDTLIGNNOFOGYGVDLIKELADKLGFNFTFRDGGNDYGSFNKTTNSTSGMLKEIVEGRADLAITDLTITSEREEVIDFSIPFMNLGIA TLVVTTILENPYVMRRPNFQALSGNERFEGFCVDMLRELAELLRFRYRLRLVEDGLYGAPEPNGSWTGMVGELINRKADLAVAAFTITAEREKVIDFSKPFMTLGIS

\section{TM2}

ILYVKPQKAPPALFSFMDPFSSEVWLYLGIAYLGVSLCFFIIGRLSPIEWDNPYPCIEE-PEELENQFTINNSLWFTTGALLQQGSEIAPKALSTRTISAIWWFFTL ILYRVHMGRKPGYFSFLDPFSPAVWLFMLLAYLAVSCVLFLAARLSPYEWYNPHPCLRARPHILENQYTLGNSLWFPVGGFMQQGSEIMPRALSTRCVSGVWWAFTL

TM3

IMVSSYTANLAAFLTIENPTSPINSVKDLADNKDDVOYGAKRTGSTRNFFSTSEEPIYIKMNEYLN - AHPEMLMENNOOGVDKVKSGTKYAFLMESTSIEFNTVREC II ISSYTANLAAFLTVQRMEVPVESADDLADQTN - IEYGTIHAGSTMTFFQNSRYQTYQRMWNYMQSKQPSVFVKSTEEGIARVLN - SRYAFLLESTMNEYHRRLNC

TM4

NLTKVGDPLDEKGYGIAMVKNWPYRDKFNKALLELQEQGVLARLKNKWWNEVGAGVCSAKSDDDGPSELGVDNLSGIYVVLVIGSIISIIISILCWCYFVYKKAKNY NLTQIGGLLDTKGYGIGMPLGSPFRDEITLAILQLQENNRLEILKRKWWEGG - - - - RCPKEEDHRAKGLGMENIGGIFIVLICGLIIAVFVAVMEFIWSTRRSAESE

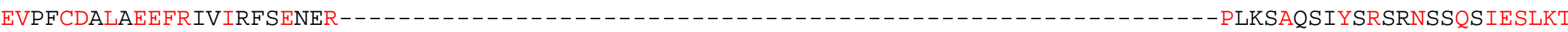
EVSVCQEMLQELRHAVSCRKTSRSRRRRRPGGPSRALLSLRAVREMRLSNGKLYSAGAGGDAGSAHGGPQRLLDDPGPPSGARPAAPTPCTHVRVCQECRRIQALRA DSE - - - - - ENMPVED - - - - . - . - . - . - SGAGAPPRGLGVPAEATSPPRPRPGPAGPRELAEHE 
(Figure 1-5b cont.)

GluR-IIE MFFNHFVILWSLFSIHISVNWAQYENFGGYDNYQSLESVPIGLLTDQNTEQMNIVFDHAIDVANQEVGTSLTSLKEEVNYGDAYQSYGKLCRMLETGIAGVFGPSSR

- HTAVHLMSICDAMDIPHIYSYMSEN - - - - - - AEGFNLHPHPADLAKALYSLITEFNWTRFIFLYESAEYLNILNELTTMLGKSGTVITVLRYDMQLNGNYKQVLR PASASTVSHICGEKEIPHIKVGPEETPRLQYLRFASVSLYPSNEDVSLAVSRILKSFNYPSASLICAKAECLLRLEELVRGFLISKETLSVRMLDDSRDP - - TPLLK

RVRKSVDNRIVVVGSSETMPEFLNQAQQVGI INEDYKYIIGNLDFHSFDLEEYKYSEANITGLRLFSPEKMAVKELLMKLGYPTDQDEFRNGSCPITVEMALTYDAV EIRDDKVSTIIIDANASISHLILRKASELGMTSAFYKYILTTMDFPILHLDGIVEDSSNILGFSMFNTSHPFYPEFVRSLNMSWRENCEASTYLGPALSAALMFDAV

QLFAQTLKNLPFKPMP - QNCSQRTESVRDDGSSFKNYMRTLRLTDRLLTGPIYFEGN - VRKGYHLDVIELQPSGIVKVGTWDEDRQYRPQRLAPTTAQFDSVDNSL HVVVSAVRELNRSQEIGVKPLACTSANIWPHGTSLMNYLRMVEYDG - - LTGRVEFNSKGQRTNYTLRILEKSRQGHREIGVWYSN - - - RTLAMNATTLDIN - LSQTL

ANKTFIILLSVPNKPYAQLVETYKQLEGNSOYEGYGVDLIKELADKLGFNFTFVNGGNDYGSYNKSTNESTGMLREIMTGRADLAITDLTITSEREOALDFTIPFMN ANKTLVVTTILEN - PYVMRRPNFQALSGNERFEGFCVDMLRELAELLRFRYRLRLVEDGLYGAPEPNGSWTGMVGELINRKADLAVAAFTITAEREKVIDFSKPFMT

\section{TM2}

LGIAILYLKPQKATPELFTFMDPFSEEVWWFLGFSFLGVSLSFFILGRLSPSEWDNPYPCIEE-PEELENOFTLGNSIWFTTGALLQQGSEIGPKALSTRTVASFWW LGISILYRVHMGRKPGYFSFLDPFSPAVWLFMLLAYLAVSCVLFLAARLSPYEWYNPHPCLRARPHILENQYTLGNSLWFPVGGFMQQGSEIMPRALSTRCVSGVWW

TM3

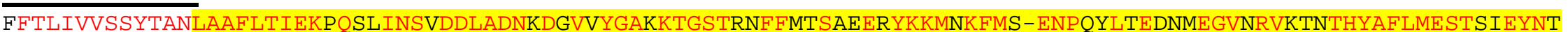
AFTLI I ISSYTANLAAFLTVQRMEVPVESADDLADQTN - IEYGT IHAGSTMTFFQNSRYQTYQRMWNYMQSKQPSVFVKSTEEGIARVLN - SRYAFLLESTMNEYHR

\section{TM4}

KRECNLKKIGDALDEKGYGIAMRKDWPHRGKFNNALLELQEQGVLEKMKNKWWNEVGTGICATKEDAPDATPLDMNNLEGVFFVLLVGSCCALLYGIISWVLFVMKK RLNCNLTQIGGLLDTKGYGIGMPLGSPFRDEITLAILQLQENNRLEILKRKWWEGG - - - - RCPKEEDHRAKGLGMENIGGIFIVLICGLIIAVFVAVMEFIWSTRRS

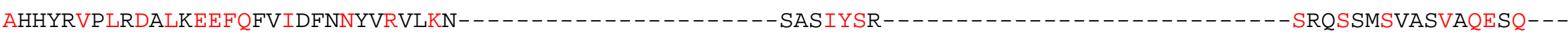
AESEEVSVCQEMLQELRHAVSCRKTSRSRRRRRPGGPSRALLSLRAVREMRLSNGKLYSAGAGGDAGSAHGGPQRLLDDPGPPSGARPAAPTPCTHVRVCQECRRIQ

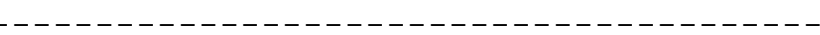

ALRASGAGAPPRGLGVPAEATSPPRPRPGPAGPRELAEHE 


\begin{tabular}{|c|c|c|c|c|c|c|c|c|}
\hline $\begin{array}{c}\text { Subunit } \\
\text { name }\end{array}$ & Protein ID & Gene ID & $\begin{array}{c}\text { Subunit } \\
\text { name }\end{array}$ & Protein ID & Gene ID & $\begin{array}{c}\text { Subunit } \\
\text { name }\end{array}$ & Protein ID & Gene ID \\
\hline GluR-IIA & NP 523484 & 33788 & CG 3814 & $\begin{array}{l}\text { NP } 610824 \\
\text { NP } 725236\end{array}$ & 36418 & CG 14586 & NP 649013 & 39983 \\
\hline GluR-IIB & NP 523485 & 33789 & CG 33513 & $\begin{array}{l}\text { NP } 001014716 \\
\text { NP } 001014715 \\
\text { NP } 001014714\end{array}$ & 31107 & CG 14585 & NP 649012 & 39982 \\
\hline $\begin{array}{l}\text { GluR-IIC } \\
\text { (GluRIII) }\end{array}$ & $\begin{array}{l}\text { NP } 608557 \\
\text { NP } 722670\end{array}$ & 33275 & CG 2902 & NP 730940 & 40665 & CG15732 & NP 572795 & 32189 \\
\hline $\begin{array}{l}\text { GluR-IID } \\
\text { (=kaiRIA) }\end{array}$ & NP 651982 & 44483 & CG 30379 & NP 724626 & 246578 & CG 6185 & NP 648455 & 39269 \\
\hline GluR-IIE & NP 001036733 & 318623 & GluR I & NP 476855 & 38742 & CG 15324 & NP 572411 & 31692 \\
\hline CG 11155 & $\begin{array}{l}\text { NP } 651941 \\
\text { NP } 726648 \\
\text { NP } 726649\end{array}$ & 43822 & GluR IB & NP 524755 & 44484 & CG 11775 & NP 649833 & 41052 \\
\hline CG 9935 & $\begin{array}{c}\text { NP } 651931 \\
\text { NP } 001036250 \\
\text { NP } 001036251\end{array}$ & 43806 & CG 17274 & NP 650923 & 42471 & CG 10633 & NP 647962 & 38616 \\
\hline $\begin{array}{c}\text { Clumsy } \\
\text { (=CG 8681) }\end{array}$ & $\begin{array}{c}\text { NP } 523610 \\
\text { NP } 001036373\end{array}$ & 35394 & CG 31718 & NP 723585 & 318912 & CG 10101 & NP 649720 & 40891 \\
\hline CG 5621 & $\begin{array}{c}\text { NP } 650927 \\
\text { NP } 001036735\end{array}$ & 42476 & CG 2657 & NP 001097043 & 33157 & CG 15627 & NP 608863 & 33683 \\
\hline CG 3822 & NP 650925 & 42473 & CG 7385 & NP 649176 & 40198 & CG 8533 & $\begin{array}{l}\text { NP } 649148 \\
\text { NP } 730452 \\
\text { NP } 730453\end{array}$ & 40157 \\
\hline CG 32704 & NP 727328 & 31867 & CG 14076 & NP 649074 & 40065 & CG 17275 & NP 650924 & 42472 \\
\hline CG 3798 & $\begin{array}{l}\text { NP } 725237 \\
\text { NP } 725238 \\
\text { NP } 523722 \\
\text { NP } 725239 \\
\text { NP } 725241 \\
\text { NP } 725240\end{array}$ & 36419 & $\begin{array}{c}\text { CG } 33492 \\
(=\text { CG 17587) }\end{array}$ & NP 995744 & 2768714 & CG 17152 & NP 648548 & 39378 \\
\hline
\end{tabular}

Table 1-1. Glutamate receptor genes in Drosophila genome. 36 different glutamate receptors genes are found in NCBI database (National Center for Biotechnology Information). 


\section{Chapter Two}

\section{Furthering Pharmacological and Physiological Assessment of the Glutamatergic Receptors at the Drosophila Neuromuscular Junction}

\section{$\underline{\text { INTRODUCTION }}$}

The function and pharmacology of glutamate receptors in general are of prime interest since they are the major excitatory receptor type used within the mammalian central nervous system (CNS). There are extensive investigations into the pharmacology, physiology, molecular structure and regulation in expression as well as turnover in mammalian preparations of glutamate receptors (Dingledine et al., 1999; Hollmann and Hainemann, 1994; Lipsky and Goldman, 2003; Mayer, 2005; Pauly et al., 2005; Seeburg, 1993; Thiagarajan et al., 2005). Earlier studies on glutamate receptors at the neuromuscular junction of insects and crustaceans paved the way to dissecting the functional aspects of these receptors within vertebrates (Anderson et al. 1976; Cull-Candy and Parker, 1983; Gration et al., 1981; Jan and Jan, 1976; Patlak et al., 1979; Shinozaki and Ishida, 1981; Shinozaki and Shibuya, 1974). Still today, invertebrate models serve a vital role in describing regulation and developmental aspects of glutamate receptor function (Featherstone et al., 2005; Guerrero et al., 2005; Littleton and Ganetzky, 2000; Logsdon et al., 2006; Pawlu et al., 2004; Rasse et al., 2005). Considering that Drosophila melanogaster is a true model organism with a known genome and used for successful rapid induction of mutations in various studies of development and synaptic mechanisms, it is important to understand the pharmacological and physiological function within this model synaptic neuromuscular preparation (Atwood et al., 1993; Betz et al. 1993; Jan and Jan 1976; Kurdyak et al., 1994; Li et al., 2002; Li and Cooper, 2001; Pawlu et al., 2004; Ruffner et al., 1999; Sigrist et al., 2002, 2003; Stewart et al., 1994, 1996).

The molecular sequence of the glutamate receptors on the Drosophila skeletal muscle has been described; however, to date there are few detailed investigations on the pharmacological profile of the intact larval Drosophila NMJ (Chang and Kidokoro, 1996; Chang et al., 1994; Delgado et al., 1989; Schuster et al., 1991; Zhang et al., 1999). Glutamate receptors are defined as NMDA (Nmethyl-D-aspartic acid) and non-NMDA subtypes (i.e. AMPA, kainate and $\delta$ ) based on their pharmacological sensitivity activating the receptors. The molecular sequences of the glutamate receptors for Drosophila NMJs were performed on the body wall muscles in larvae by isolating the skeletal muscle and the body wall (skeletal muscles attach to the cuticle body wall). The total mRNA was extracted from this muscle tissue and probed against an "affymetrix Drosophila genechip". A number of Drosophila glutamate receptor subunits were found to be present. The sequences isolated were identified by comparison to whole larval DNA known sequences for glutamate receptors. The ones identified 
are GluRIIA, GluRIIB, GluRIIC and GluRIID. GluRIIE was later isolated when real-time PCR was used to follow up on the amplified mRNA of the four receptor subtypes in quantification of expression levels. The expression profile of the five glutamate receptor subunits has been conducted in body wall muscle by in situ hybridization for embryos and larvae (Guerrero et al., 2005; Marrus et al., 2004).

With the RT-PCR and the genomic profiling the sequence indicates that there are 5 glutamate receptor subunits (DGluR-IIA, DGluR-IIB, DGluR-IIC, DGluR-IID, DGluR-IIE) expressed in skeletal muscle and all of them are ionotropic receptors. In comparing the molecular sequence of these 5 muscleexpressed glutamate receptors with vertebrates, they showed the most similarity to the vertebrate kainate/AMPA receptor subtypes; therefore, they have been defined as a kainate receptor subtype without pharmacological examination (Betz et al., 1993; Guerrero et al., 2005; Marrus et al., 2004; Qin et al., 2005; Völkner et al., 2000). However, a recent physiological and pharmacological study demonstrates that the responses are primarily of a quisqualate subtype and not a kainate or an AMPA subtype since the muscle is not depolarized by kainate or AMPA at concentrations as high as $1 \mathrm{mM}$ (Bhatt and Cooper, 2005). In fact, it appears that kainate might block the glutamate receptors since the excitatory postsynaptic potential (EPSP) is reduced in the presence of kainate (on average by $40 \%$ for $1 \mathrm{mM}$ and $500 \mu \mathrm{M}$ but about $20 \%$ for $100 \mu \mathrm{M}$ ). The reduction in the EPSP amplitude occurred without any significant change in resting membrane potential; thus, kainate did not depolarize the muscle as observed for application of glutamate or quisqualate (Bhatt and Cooper, 2005).

In addition, the procedures used to identify the molecular sequence of the glutamate receptors at the NMJ, by homogenizing the skeletal muscle and body wall, did not allow for assessment for potential presynaptic glutamate receptors. In the past procedure, the CNS was removed and thus the cell bodies of the motor neurons were not incorporated in the assay. Considering that there are presynaptic glutamatergic receptors for neurons in the vertebrate CNS (Park et al., 2006) and at crayfish NMJs (Schramm and Dudel, 1997), there may well be pre-synaptic autoreceptors present at the Drosophila NMJ. Crayfish NMJs show many similarities to the larval Drosophila NMJs (Atwood and Cooper, 1995, 1996) which is not so surprising since they are both arthropods. However, mixed results for the glutamatergic autoreceptors were obtained at the crayfish NMJ in that the responses depended on the depolarized state of the nerve terminal for the facilitatory action of exogenously applied glutamate and for the metabotropic agonist t-ACPD (Schramm and Dudel, 1997). In addition, some preparations showed a depression of presynaptic release while yet other preparations exhibited excitation at the same concentration of t-ACPD. Regardless of the response at the crayfish NMJ, there appears to be one or more types of presynaptic, glutamatergic receptors for the crayfish preparation (Schramm and Dudel, 1997). The earlier observation of the rapid reduction in the EPSP by kainate at the Drosophila NMJ is most likely due to one or both of the following factors: (1) antagonist action on the postsynaptic glutamate receptors; (2) reducing the number of presynaptic vesicular fusion events. It is possible both mechanisms might occur. Direct blocking action on postsynaptic receptors is a 
possibility for kainate as many known agonists can be antagonists for subtypes within a receptor family (Chang and Weiss, 2002; Eberle et al., 2001; Hruby et al., 2003). This is likely as a part of the extracellular receptor domains for glutamate receptors must be similar in order to bind glutamate. However, at the $3^{\text {rd }}$ instar Drosophila NMJ the binding of kainate does not transduce activation of the glutamate ligand gated ion channels (Bhatt and Cooper, 2005; Dudel et al. 1992; Heckmann and Dudel, 1997). There is also precedence for kainate, a potent agonist for some vertebrate metabotropic receptors, to act presynaptically in reducing evoked transmitter release via autoreceptors on the presynaptic terminals (Chittajallu et al., 1996; Frerking et al., 2001; Kamiya and Ozawa, 1998; McLarnon and Quastel, 1988; Park et al., 2006). Kainate is known to bind metabotropic glutamate receptors (Zhang et al., 1999) and have a presynaptic action in altering transmitter release from presynaptic terminals in vertebrates (Kamiya and Ozawa, 2000; Kamiya et al., 2002; Park et al., 2006). In rats, kainate and the kainate receptor agonist, ATPA, act presynaptically in reducing glutamate release from primary sensory neurons (Kerchner et al., 2001). However, in the rat neocortex, kainate receptors have been demonstrated to have both facilitatory as well as inhibitory actions due to dose dependent effects. The mechanism proposed for the biphasic effect is that low doses excite the presynaptic terminal while high doses have an inhibitory effect on the postsynaptic target (Campbell et al., 2007). Thus, depending on the type of preparation and dosage, kainate might excite or inhibit the presynaptic terminal. The evidence suggests a mechanism of action via kainate-sensitive glutamatergic autoreceptors through a metabotropic action within the presynaptic terminals or by direct action on the presynaptic voltage-gated $\mathrm{Ca}^{2+}$ channels (see reviews Lerma, 2003 and Nicoll et al., 2000). In addition, the actions of glutamate at newly hatched Drosophila larvae revealed presynaptic actions in enhancing the occurrences of spontaneous events without any alteration in the amplitude of the quantal currents (Zhang et al., 1999). The mechanisms suggested for this phenomenon are that presynaptic autoreceptors are present which enhances synaptic transmission. In addition, the pharmacological agonist (1S, 3S)-ACPD for mGluR mimicked the effect of glutamate (Zhang et al., 1999). This helps to establish that autoreceptors at this NMJ are likely in the $3^{\text {rd }}$ instar and that there maybe developmental differentiation in presynaptic receptor subtypes to account for the depressing action of kainate in the $3^{\text {rd }}$ instar.

The purpose of this study is to test for these possibilities. I have approached this by accessing the characteristics of single quanta (i.e., peak amplitude) and frequency of spontaneous events with intracellular measures of miniature EPSPs (mEPSPs) from the whole muscle. I also examined further pharmacological profiles of the Drosophila NMJ to build knowledge of this model synaptic preparation.

\section{METHODS}

\section{Electrophysiology and staging of flies}


All the electrophysiology and staging of larvae are routine procedures and have been described previously. The dissection technique was previously reported (Ball et al., 2003; Campos-Ortega and Hartenstein, 1985; Li et al., 2002). The physiological saline is $\mathrm{HL} 3$ (in $\mathrm{mM}$ ): $1.0 \mathrm{CaCl}_{2} 2 \mathrm{H}_{2} \mathrm{O}, 20 \mathrm{MgCl}_{2}, 70$ $\mathrm{NaCl}, 5 \mathrm{KCl}, 10 \mathrm{NaHCO}_{3}, 5$ trehalose, 115 sucrose, $5 \mathrm{BES}$ (N,N-bis[2-hydroxyethyl]-2-aminoethanesulfonic acid) and adjusted to a pH of 7.2 (Stewart et al., 1994). All experiments were performed at room temperature $\left(20-21^{\circ} \mathrm{C}\right)$. The recording techniques have been previously described for intracellular EPSP measures (Dasari and Cooper, 2004; Sparks et al., 2004; Stewart et al., 1994) and extracellular focal quantal recordings (Cooper et al., 1995a; Harrison and Cooper, 2003). The compound amplitude of the excitatory postsynaptic potentials (EPSP) elicited by Is and Ib motor nerve terminals in segment 3 of muscle m6 were monitored as in Kurdyak et al., (1994) and Ruffner et al., (1999). The identified $\mathrm{m} 6$ muscle was used in each preparation. Only preparations with a resting membrane potential of $-50 \mathrm{mV}$ or greater were used and the HL3 dissection medium was completely replaced by HL3 media containing the pharmacological compounds.

\section{Quantal analysis}

All quantal analysis of intracellular whole muscle recordings were as described previously (Cooper et al., 1995b). The quantal analysis of measuring spontaneous frequency and characteristics of the quantal shape are standard approaches.

\section{Pharmacology}

In an earlier report (Bhatt and Cooper, 2005), actions of NMDA, AMPA, Lglutamate, kainate, quisqualic acid, NBQX, AP5, and DNQX, with regard to synaptic transmission and direct effects on the muscle fibers, was examined. This study showed that kainate reduced evoked EPSPs. The same procedures for exchanging the bathing medium with pharmacological agents were used. The compound trans-( \pm )-1-Amino-1,3-cyclopentanedicarboxylic acid monohydrate (tACPD) is a known mGluR agonist (Holscher et al., 1997) and has been shown to have some effect on newly hatched Drosophila larvae (Zhang et al. 1999). I examined the actions of ATPA, a known agonist of the kainate receptor in vertebrates, and 1, 3-ACPD on synaptic responses. The ATPA, domoic acid and the trans-( \pm -1-Amino-1,3-cyclopentanedicarboxylic acid monohydrate (t-ACPD) were obtained from Sigma

\section{Statistical analysis}

A non-parametric analysis (Wilcoxon rank sum) or a parametric Student's $t$-test were used for assigning a significance at a $p<0.05$ depending on the appropriate experimental design as explained in the RESULTS section.

\section{Sequence Comparisons}

All of the glutamate receptor amino acid sequences used are annotated in National Center for Biotechnology Information (NCBI) database. There are 36 
different genes encoding 36 different kinds of glutamate receptor subunits in Drosophila melanogaster. Among them, five subunits consisting of postsynaptic receptors were compared to five different kainate type glutamate receptor subunits in Homo sapiens. Both whole protein and extracellular domain sequences of each subunit were aligned to show the similarity and calculate the statistical matches between two species, respectively. The bl2seq in NCBI and Clustal W were used to align two different protein sequences. In Clustal W the default settings were used except ITERATION to alignment and NUMERITER were set to 3 .

\section{RESULTS}

In measuring the evoked EPSPs and spontaneous quantal events a 3rd instar larvae is filleted so that each segment is observed. By stimulating a selected segmental nerve, only one nerve root is recruited which is important since the muscle fibers are electrically coupled between segments. Various identified muscles with the rather simplistic innervation profiles are shown in Figure 2-1A. In these studies, I utilized muscle $6(\mathrm{~m} 6)$ because of the well characterized innervation as depicted in Figure 2-1B (Atwood et al., 1993; Kurdyak et al., 1994; Li et al., 2002). The synaptic properties of the Is and Ib motor nerve terminals have been previously described (Atwood et al., 1993; Kurdyak et al., 1994; Li et al., 2002). I obtained similar measures as earlier reported. I did ensure to observe all three sized EPSP events (Ib, Is, and Ib+ls) in order to subsequently monitor the compound Ib+ls EPSP. The resting membrane potential was also monitored and only preparations which did not appear damaged or with a resting membrane potential more negative than $-50 \mathrm{mV}$, in normal physiological saline, were used for assessing the effects of various pharmacological agents.

Application of a saline containing kainate $(1 \mathrm{mM})$ rapidly reduces the EPSP amplitude and the effect can be partially reversed by extensive exchanging the bathing media back to normal saline (Figure 2-2A). The extant in the reduction of the EPSP amplitude is dose-dependent, with an almost $50 \%$ reduction at $1 \mathrm{mM}$ (Figure 2-2B). All three concentrations produced a significant reduction in the EPSP amplitudes $(n=6$ for each concentration; $p<0.05$ Wilcoxon rank sum nonparametric). There is no significant effect on the resting membrane potential on m6 for any of the three concentrations tested (Figure 2-2C). However when considering sham control experiments of exchanging the bathing medium with saline and providing the same time period in which EPSP measure were made there is run down in the EPSP amplitude by $13.5 \%$ (Figure 2-2D). The individual values are shown next to the mean value. The sham procedures produced a significant effect in reducing the EPSP responses $(n=6 ; p<0.05$ Wilcoxon rank sum non-parametric). The mean value was not subtracted from experimental conditions with pharmacological agents in the remaining results, but this effect should be considered. Thus, exposure to $500 \mu \mathrm{M}$ and $1 \mathrm{mM}$ kainate produced a significant difference from sham control, whereas $100 \mu \mathrm{M}$ did not $(p<0.05$ Student's $t$-test). 
In examining the effects of the AMPA and kainate receptor agonist, domoic acid $(10 \mu \mathrm{m}$ and $1 \mathrm{mM})$, the postsynaptic receptor sensitivity to spontaneous quantal responses and the frequency of spontaneous events were assessed. It was readily apparent that domoic acid at $1 \mathrm{mM}$ rapidly reduced the amplitude of spontaneous mEPSP quantal events (Figure 2-3A) as well as the frequency of occurrences (Figure 2-3B). Domoic acid at $10 \mu \mathrm{M}$ or $1 \mathrm{mM}$ did not produce an effect on the resting membrane potential (Figure 2-3C). Glutamate at $10 \mathrm{mM}$ produced similar effects in reducing the mEPSP amplitude and frequency but this was compounded by the rapid depolarization that glutamate produced $(p<0.05$; Figure 2-3C). The differences in the AMPA and kainate receptor agonist and glutamate are that, domoic acid did not significantly depolarize the muscle; however, the mEPSPs were still affected. The combination of domoic acid $(1 \mathrm{mM})$ and glutamate $(10 \mathrm{mM})$ produced a reduced depolarization of the muscle and as expected, a reduced mEPSP amplitude and frequency occurred (Figure 2-3B, C). These results suggest domoic acid is an antagonist to the postsynaptic glutamate receptors. The effect on the reduced frequency of the mEPSPs is due to the gradual reduction in the mEPSP amplitude, such that they are not discernable from noise in the baseline and thus are not detected to monitor their frequency.

Similar results were obtained for domoic acid as for kainate. The mEPSP amplitudes and the frequency are reduced (Figure 2-4A, B). Also the resting membrane potential is not significantly altered. In examining subtle effects on single quantal events the shape of the quantal events were examined and significant effects in rise time or decay were measured (data not shown). The amplitude and area (i.e., area under the curve) of the mEPSPs both decreased in a dose-dependent manner for $1 \mathrm{mM}$ and $500 \mu \mathrm{M}$ (Figure 2-4C). With exposure to kainate, the smaller mEPSPs appeared to fade into the background noise while the previously larger mEPSPs events were now observed as smaller mEPSPs.

Since the postsynaptic glutamate receptors on the muscle appear to bind kainate, I expected the known agonist of kainate receptors, ATPA, to act similarly, despite knowing that the receptors are not a kainate subtype. ATAP at $1 \mathrm{mM}$ did not reduce the evoked EPSPs (Figure 2-5A, B) nor did ATPA have any effect on the resting membrane potential (Figure 2-5C). In fact, some preparations increased in the EPSP amplitude while others decreased, thus producing no net significant effect.

If there are subtle effects of kainate in depressing presynaptic vesicular docking or altering $\left[\mathrm{Ca}^{2+}\right]_{i}$ dynamics, one would expect that facilitation or the short-term depression (i.e., negative facilitation) index might detect such an effect. A short four pulse train of $20 \mathrm{~Hz}$ produces the characteristic response known for this preparation, as shown in Figure 2-6A. The initial EPSP is normally used as a relative reference to the $4^{\text {th }}$ EPSP in the train. It has been previously demonstrated that $1 \mathrm{mM}\left[\mathrm{Ca}^{2+}\right]_{0}$ will produce a marked depression in the response train where as $500 \mu \mathrm{M}\left[\mathrm{Ca}^{2+}\right]_{\mathrm{o}}$ produces less depression and sometimes a slight facilitation. Thus, both $1 \mathrm{mM}$ and $500 \mu \mathrm{M}\left[\mathrm{Ca}^{2+}\right]_{0}$ were examined with $1 \mathrm{mM}$ and $500 \mu \mathrm{M}$ kainate to unmask any ceiling effect in the depression by $1 \mathrm{mM}$ $\left[\mathrm{Ca}^{2+}\right]_{0}$ exposure. The $1^{\text {st }}$ and $4^{\text {th }}$ EPSPs rapidly attenuate in amplitude upon exposure to $1 \mathrm{mM}$ kainate and $0.5 \mathrm{mM}\left[\mathrm{Ca}^{2+}\right]_{0}$ (Figure 2-6B). The EPSPs also 
show a large reduction to kainate when bathed with $1 \mathrm{mM}\left[\mathrm{Ca}^{2+}\right]_{\mathrm{o}}$ (Figure 2-6C). However, no significant differences, by parametric analysis (Student's $t$-test), in the facilitation index are observed among the four conditions for exposure to kainate as determined by the difference in the median FI values. The difference is measured from saline to exposure of kainate within each preparation $(n=5$; $500 \mu \mathrm{M}$ kainate- $1 \mathrm{mM}\left[\mathrm{Ca}^{2+}\right]_{0} ; 1 \mathrm{mM}$ kainate- $1 \mathrm{mM}\left[\mathrm{Ca}^{2+}\right]_{0} ; 500 \mu \mathrm{M}$ kainate-500 $\mu \mathrm{M}$

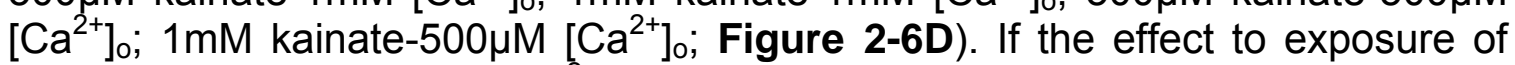
kainate $(1 \mathrm{mM})$ with $1 \mathrm{mM}\left[\mathrm{Ca}^{2+}\right]_{0}$ is analyzed by non-parametric statistics, then five out five preparations showed an increase in the mean $\mathrm{FI}(p<0.05$, Wilcoxon Rank Sum). However, two preparations showed only small increases of 10 and $12 \%$. Thus, I do not feel confident in stating that there is a significant increase in the mean FI with kainate by non-parametric analysis. On the other hand, boxwhisker plots of the FI values before and after exposure to kainate, for each preparation, in the various paradigms revealed a trend again related to the same data set of kainate $(1 \mathrm{mM})$ with $1 \mathrm{mM}\left[\mathrm{Ca}^{2+}\right]_{\mathrm{o}}$ (Figure 2-7). The box-whisker plots represent the median, $25^{\text {th }}, 75^{\text {th }}$, and range. One can observe that the variability in the distributions is larger for the kainate $(1 \mathrm{mM})$ with $1 \mathrm{mM}\left[\mathrm{Ca}^{2+}\right]_{\mathrm{o}}$ as compared to the same preparations before the exposure. In the other paradigms the variability is about the same for saline and kainate exposure. To further examine this variability in $\mathrm{FI}$, the standard deviation for each preparation before and after exposure to kainate was examined. Only the kainate $(1 \mathrm{mM})$ with $1 \mathrm{mM}\left[\mathrm{Ca}^{2+}\right]_{0}$ showed a consistent trend (five out of five; $p<0.05$, Wilcoxon Rank Sum Test; also by Student's $t$-test $p<0.05$ ) with an increase in the variability while exposed to kainate as compared to the saline baseline. The changes in the individual preparations for this paradigm are shown in Figure 2-8.

In order to further investigate possible presynaptic, metabotropic glutamate receptor action on the motor nerve terminals, the common metabotropic glutamate receptor agonist trans-1-aminocyclopentane-1, 3dicarboxylic acid (t-ACPD) was used. Potentially, an increase or a decrease in the evoked EPSP would be possible at this insect NMJ since at the crayfish NMJ (also an arthropod species) an effect was reported (Schramm and Dudel, 1997). However, no significant alterations on evoked EPSPs amplitudes (Figure 2-9A, $B$ ) or on the resting membrane potential of the muscle occurred during exposure to t-ACPD $(500 \mu \mathrm{M})$ at the Drosophila NMJ. In comparison to the sham saline control (Figure 2-2D), the distributions in EPSP amplitudes over lap; thus, the slight reduction in EPSP amplitudes with t-ACPD are not significant.

\section{Discussion}

In this study I showed, in a concentration-dependant manner, that kainate rapidly reduced the amplitude of evoked EPSPs as well as the spontaneous quantal events. The lack in any response of t-ACPD and ATPA as well as a lack in these compounds mimicking kainate's action suggest that there is not a presynaptic mechanism of a kainate-like or metabotropic autoreceptors on these motor nerve terminals. However, the kainate receptor agonist, domoic acid, did follow the actions of kainate in reducing the evoked EPSPs. I suggest this action 
is due to domoic acid and kainate acting as an antagonist on the postsynaptic quisqualate receptors present on muscle and that no presynaptic contribution can be proposed for the rapid reduced amplitude in the evoked EPSP and quantal responses. The reduced frequency in the spontaneous quantal responses in the presence of kainate or domoic acid appears to be due to the responses decreasing in size to a point that they are not detectable from the noise or that the postsynaptic receptors are fully blocked.

The responses observed at the crayfish NMJ by Schramm and Dudel (1997) for analogous types of NMJs as I used in the Drosophila larval NMJ (Atwood and Cooper, 1995, 1996) must be different in regards to presynaptic autoreceptors. In their study, t-ACPD and glutamate itself produced varied responses in mean quantal content. Some preparations they reported showed an enhancement while others a decrease in evoked postsynaptic events. They concluded that there may be differential regulation in the type and density of the metabotropic presynaptic autoreceptors to account for variation among preparations and effects to t-ACPD and glutamate. There are methodological differences in stimulating the nerve terminals. In my studies, I elicited action potentials within the nerve to result in $\mathrm{Ca}^{2+}$ influx and vesicular fusion. However, Schramm and Dudel used depolarization pulses delivered over the terminal, through the focal electrode, to directly open voltage-gated channels. Seal stability with the focal electrode over the NMJ, longer than normal action potential depolarization pulses and tweaking the current pulses to a fine degree in order to balance a percentage of activating voltage dependent ion channels, would appear to induce potentially more experimental variability among preparations than inducing a standard action potential within the nerve for evoking synaptic transmission. The large field effect of a current pulse, to induce presynaptic depolarization, through a macropatch electrode could have an effect on the postsynaptic glutamate receptors such that the receptor interaction with pharmacological agents of even glutamate itself might be altered. To resolve these issues in the crayfish would require selectively stimulating the motor nerve and repeating the study.

As for domoic acid, the results suggest kainate is an antagonist to the postsynaptic glutamate receptors in a dose-dependent manner. It appears kainate can behave as an antagonist but not ATPA for these postsynaptic muscle receptors. In addition, since ATPA did not produce any significant alteration in the evoked EPSP amplitude, there does not appear to be any pre- or postsynaptic action of ATPA. Quisqualate has been suggested to activate ionotropic as well as metabotropic glutamate receptors just as glutamate does itself, which suggests quisqualate is a non-selective glutamate receptor agonist (Schoepp et al., 1990). However, AMPA, which is significantly similar to quisqualate in structure and is a weak kainate receptor agonist, cannot activate nor even bind to homomeric GluR6 kainate receptor (Egebjerg et al., 1991). Such differences may be induced by steric hindrance near the ligand binding domain. In fact, AMPA did not change the EPSP amplitude in my studies, but kainate resulted in reduced amplitude by acting as an antagonist. Therefore, there seems to be other factors besides steric hindrance. It would seem likely that a ligand binding site of kainate 
at the Drosophila NMJ would be highly conserved to the vertebrate kainate receptor, since kainate is blocking evoked responses; however, the similarity of extracellular domains are not very high. Recent studies suggested that the properties of ionotropic glutamate receptor subunits can be changed by mutating the amino acid at the second transmembrane segment which forms the lining of the pore region (Bochet et al., 1991; Curutchet et al., 1992; Verdoorn et al., 1991). For the functional muscle-expressed glutamate receptor GluR-IIC, GluRIID, GluR-IIE and either GluR-IIA or GluR-IIB is essential for function. Therefore, only based on the ligand binding domain tetrameric structure was suggested. The correct subunit stoichiometry is not known. The receptor can be pentameric or higher such as (IIA)(IIB)(IIC)(IID)(IIE) or (IIA)(IIA)(IIC)(IID)(IIE) to be functional. Also, glutamate receptor complexes which contain either GluR-IIA or GluR-IIB have opposite physiological properties by showing slow and fast desensitization, respectively (DiAntonio et al., 1999). Thus, as constituted receptor subunits are put together it is likely that the receptor is functionally changed. There could also be mixed subunit combinations along the nerve terminal among the many synaptic sites, since the terminal is developmental different in age along its length (Atwood and Cooper, 1995; He et al., 2009; Li et al., 2002) and function (Bogdanik et al., 2004; Guerrero et al., 2005; Marrus et al., 2004; Liebl and Featherstone, 2005;). Developmental alteration in kainate receptors are known in vertebrates (Lee et al., 2001). Thus, variability in whole muscle responses to pharmacological agents might be masked, but if older and younger regions of the terminals were monitored with focal macropatch electrodes larger differences in the pharmacological profiles and sensitivities might be observed.

It was proposed that the five subunits consisting of postsynaptic receptors in Drosophila NMJ are somewhat similar in homology to kainate receptors in vertebrates and thus, they were termed kainate-like (Betz et al., 1993; Guerrero et al., 2005; Marrus et al., 2004; Qin et al., 2005; Völkner et al., 2000). In comparing the postsynaptic receptor molecular sequence of Drosophila NMJ with a kainate receptor in Homo sapiens, which contains the highest homology in the vertebrates, each subunit shows a moderate similarity between two species. Extracellular domains of the receptors are not significantly higher in similarity compared to total sequence (Figure 2-10). In a phylogeny tree with 36 glutamate receptor protein sequences in Drosophila melanogaster and human's NMDA, kainate and AMPA receptors, the five subunits of postsynaptic receptors in Drosophila NMJ appear to be in a different subgroup to even the closest of the vertebrate kainate/AMPA type receptors (Figure 2-11).

There are multiple examples of pre- and/or post-synaptic action of kainate in enhancing or suppressing synaptic transmission in vertebrate neuronal circuits (Grabauskas et al., 2007; Hegarty et al., 2007; Kamiya and Ozawa, 2000; Pinheiro and Mulle, 2008; Rozas et al., 2003; Schoepp, 2002); and it has been reported earlier that kainate blocked synaptic transmission at the Drosophila NMJ (Chang and Kidokoro, 1996; McLarnon and Quastel, 1988). However, the earlier studies with Drosophila did use Schneider's Drosophila medium which is known to have a depressing action on synaptic transmission itself (Ball et al., 2003; Stewart et al., 1994). Mechanistically I do not know how kainate is blocking 
transmission, but it appears likely that kainate acts as an antagonist in this preparation. Possible kainate could act on the receptor to desensitize it to glutamate or in addition act as a non-competitive antagonist. Quisqualate receptors are known to be prone to desensitization at the crayfish NMJ (Tour et al., 2000) as well as other glutamate receptors (Sun et al., 2002).

The results with kainate at different concentrations and the two concentrations of bathing $\left[\mathrm{Ca}^{2+}\right]_{0}$ is interesting since only the one condition of $1 \mathrm{mM}$ kainate and $1 \mathrm{mM}\left[\mathrm{Ca}^{2+}\right]_{0}$ produced a significant trend in both an increase in the median $\mathrm{FI}$ value as well variability in the responses as compared to baseline saline exposure (Figures 2-7 and 2-8). As stated earlier the increase in median values is small in some preparations so I am cautious in bearing too much emphasis on this result; however, the increase in variability when exposed to kainate is of interest. If kainate is acting as a competitive antagonist then it is feasible to speculate with each pulse in the stimulus train the glutamate is resulting in a greater displacement of the kainate and possibly resulting in more competitive-competition. As the glutamate is being taken back up in the nerve terminal by the glutamate transporters, kainate's action can dominate producing very variable responses on the amplitudes of the EPSPs throughout the four pulse train. Considering when saline is exchanged for saline containing kainate, the $1^{\text {st }}$ EPSP within the train is dampened significantly by the antagonistic action and during the evoked release of glutamate then the kainate is displaced would possible explain the slight, but consistent, increase in the median FI values as well for this experimental paradigm. This also follows for the concept that when the $\left[\mathrm{Ca}^{2+}\right]_{0}$ is reduced to $0.5 \mathrm{mM}$, the evoked amount of glutamate is not as pronounced so less displacement of the kainate would occur, which might explain why there is not as change on $\mathrm{Fl}$ or on the median as well as the variability in the $\mathrm{FI}$ (Figure 2-7). I am not aware of reports addressing pharmacological examination of competitive antagonists during pulse trains to account for fluctuation in facilitation measures to compare my findings. More studies are warranted to examine this tentative working model in competitive antagonism during synaptic facilitation/depression to explain the mechanistic details behind these findings.

Since I have not been able to detect any presynaptic autoreceptor function at these NMJs, it would be interesting to know if these motor neurons produce mRNA for any glutamate receptor subtype. There has yet to be investigation of possible presynaptic actions at other NMJs in the larvae or adult of Drosophila. The subset of junctions that I examined may not be indicative of all the Drosophila NMJs, so further investigations are warranted. 


\section{REFERENCES}

\section{Chapter 1}

Atwood, H.L., Govind, C.K., Wu, C.F., 1993. Differential ultrastructure of synaptic terminals on ventral longitudinal abdominal muscles in Drosophila larvae. J. Neurobiol.24, 1008-1024.

Bennett MR, Pettigrew AG. The formation of synapses in amphibian striated muscle during development. J Physiol. 1975 Oct;252(1):203-39. Berridge M., 1998. Neuronal calcium signalling. Neuron.21, 13-26.

Betz, H., Schuster, C., Ultsch, A., Schmitt, B., 1993. Molecular biology of ionotropic glutamate receptors in Drosophila melanogaster. Trends in Pharmacol. Sci. 12, 428-431.

Bhatt, D., Cooper, R.L., 2005. The pharmacological and physiological profile of glutamate receptors at the Drosophila larval neuromuscular junction. Physiol. Entomol.30, 305-310.

Desai-Shah, M. and Cooper, R.L. 2009. Variable means in Ca2+ regulation which influences synaptic transmission: Comparisons between Crayfish and Drosophila NMJs. (In Press, SYNAPSE)

Dudel J. 1965. Potential changes in the crayfish motor nerve terminal during repetitive stimulation. Pflüegers Arch 282:323-337.

Dudel J. 1981. The effect of reduced calcium on quantal unit current and release at the crayfish neuromuscular junction. Pflüegers Arch 391:35-40.

Dudel J, Parnas I, Parnas H. 1983. Neurotransmitter release and its facilitation in crayfish muscle. VI. Release determined by both, intracellular calcium concentration and depolarization of the nerve terminal. Pflüegers Arch 399:1-10.

Fatt P, Katz B. 1953a. Distributed "End Plate Potentials" of Crustacean Muscle Fibers. J Exp Biol 30:433-439.

Fatt P, Katz B. 1953b. The electrical properties of crustacean muscle fibers. J Physiology 120:171-204.

Guerrero, G., Reiff, D.F., Agarwal, G., Ball, R.W., Borst, A., Goodman, C.S., Isacoff, E.Y., 2005. Heterogeneity in synaptic transmission along a Drosophila larval motor axon. Nature Neuroscience 8, 1188 - 1196.

Jan, L.Y., Jan, Y.N., 1976. Properties of the larval neuromuscular junction in Drosophila melanogaster. J. Physiol. 262, 189-214. 
Kurdyak, P., Atwood, H.L., Stewart, B.A., Wu, C.F., 1994. Differential physiology and morphology of motor axons to ventral longitudinal muscle in larval Drosophila. J. Comp. Neurol. 350, 463-472.

Kuromi H, Kidokoro Y. 1998. Two distinct pools of synaptic vesicles in single presynaptic boutons in a temperature-sensitive Drosophila mutant, shibire. Neuron. 1998 May;20(5):917-25

Li, H., Peng, X., Cooper, R.L., 2002. Development of Drosophila larval neuromuscular junctions: Maintaining synaptic strength. Neurosci. 115, 505-513.

Li, H., Cooper, R.L., 2001. Maintaining synaptic efficacy at the neuromuscular junction in Drosophila larva during normal development and prolonged life with the ecdysoneless mutant. Neurosci. 106, 193-200.

Littleton JT, Ganetzky B. Ion channels and synaptic organization: analysis of the Drosophila genome. Neuron. 2000 Apr;26(1):35-43

Marrus, S.B., Portman, S.L., Allen, M.J., Moffat, K.G., DiAntonio, A., 2004. Differential localization of glutamate receptor subunits at the Drosophila neuromuscular junction. J. Neurosci. 24, 1406-1415.

Miyazaki S. 1995. Calcium signalling during mammalian fertilization. Ciba Found Symp. 188:235-47.

Nudell BM, Grinnell AD. Regulation of synaptic position, size, and strength in anuran skeletal muscle. J Neurosci. 1983 Jan;3(1):161-76.

Pawlu, C., DiAntonio, A., Heckmann, M., 2004. Postfusional control of quantal current shape. Neuron 42(4), 607-618.

Qin, G., Schwarz, T., Kittel, R.J., Schmid, A., Rasse, T.M., Kappei, D., Ponimaskin, E., Heckmann, M., Sigrist, S.J., 2005. Four different subunits are essential for expressing the synaptic glutamate receptor at neuromuscular junctions of Drosophila. J. Neurosci. 25(12), 3209-3218.

Rosenmund C, Stevens CF. Definition of the readily releasable pool of vesicles at hippocampal synapses. Neuron. 1996 Jun;16(6):1197-207.

Ruffner, M.E., Cromarty, S.I., Cooper, R.L., 1999. Depression of synaptic efficacy in Drosophila neuromuscular junctions by the molting hormone (20Hydroxyecdysone). J. Neurophysiol. 81, 788-794.

Schramm, M., Dudel, J., 1997. Metabotropic glutamate autoreceptors on nerve 
terminals on crayfish muscle depress or facilitate release. Neurosci. Lett., 234, $31-34$.

Sigrist, S.J., Reiff, D.F., Thiel, P.R., Steinert, J.R., Schuster, C.M., 2003. Experiencedependent strengthening of Drosophila neuromuscular junctions. J. Neurosci. 23(16), 6546-6556.

Sigrist, S.J., Thiel, P.R., Reiff, D.F., Schuster, C.M., 2002. The postsynaptic glutamate receptor subunit DGluR-IIA mediates long-term plasticity in Drosophila. Neurosci. 22(17), 7362-7372.

Stewart, B.A., Atwood, H.L., Renger, J.J., Wang, J., Wu, C.F., 1994. Improved stability of Drosophila larval neuromuscular preparation in haemolymph-like physiological solutions. J. Comp. Physiol. A 175, 179-191.

Stewart, B.A., Schuster, C.M., Goodman, C.S., Atwood, H.L., 1996. Homeostasis of synaptic transmission in Drosophila with genetically altered nerve terminal morphology. J. Neurosci. 16(12), 3877-3886.

Sudhof TC. 2004. The synaptic vesicle cycle. Annu Rev Neurosci 27: 509-547.

Völkner, M., Lenz-Bohme, B., Betz, H., Schmitt, B., 2000. Novel CNS glutamate receptor subunit genes of Drosophila melanogaster. J. Neurochem. 75(5), 17911799. 


\section{Chapter 2}

Anderson, C.R., Cull-Candy, S.G., Miledi, R., 1976. Glutamate and quisqualate noise in voltage-clamped locust muscle fibres. Nature 261,151-153.

Atwood, H.L., Cooper, R.L., 1995. Functional and structural parallels in crustaceans and Drosophila neuromuscular systems. American Zoologist 35(6), 556- 565.

Atwood, H.L., Cooper, R.L., 1996. Synaptic diversity and differentiation: Crustacean neuromuscular junctions. Invertebrate Neurosci. 1, 291-307.

Atwood, H.L., Govind, C.K., Wu, C.F., 1993. Differential ultrastructure of synaptic terminals on ventral longitudinal abdominal muscles in Drosophila larvae. J. Neurobiol. 24, 1008-1024.

Ball, R., Xing, B., Bonner, P., Shearer, J., Cooper, R.L., 2003. Long-term maintenance of neuromuscular junction activity in cultured Drosophila larvae. Comp. Biochem. Physiol. A 134, 247-255.

Betz, H., Schuster, C., Ultsch, A., Schmitt, B., 1993. Molecular biology of ionotropic glutamate receptors in Drosophila melanogaster. Trends in Pharmacol. Sci. 12, 428-431.

Bhatt, D., Cooper, R.L., 2005. The pharmacological and physiological profile of glutamate receptors at the Drosophila larval neuromuscular junction. Physiol. Entomol. 30, 305-310.

Bochet, P., Dutriaux, A., Lambolez, B., Nalivaiko, E., Rossier, J., Prado-deCarvalho, L., 1991. A chimeric glutamate receptor subunit : discrete changes modify the properties of the channel. Biochem. Biophys. Res. Commun. 177, 1183-1187.

Bogdanik, L., Mohrmann, R., Ramaekers, A., Bockaert, J., Grau, Y., Broadie, K., Parmentier, M.L., 2004. The Drosophila metabotropic glutamate receptor DmGluRA regulates activity-dependent synaptic facilitation and fine synaptic morphology. J. Neurosci. 24(41), 9105--9116.

Campbell, S.L., Mathew, S.S., Hablitz, J.J., 2007. Pre- and postsynaptic effects of kainate on layer II/III pyramidal cells in rat neocortex. Neuropharmacol 53(1), $37-47$.

Campos-Ortega, J.A., Hartenstein, V., 1985 The embryonic development of Drosophila melanogaster. Spring-Verlag, Berlin. 
Chang, H., Ciani, S., Kidokoro, Y., 1994. Ion permeation properties of the glutamate receptor channel in cultured embryonic Drosophila myotubes. J. Physiology 476, 1-16.

Chang, H., Kidokoro, Y., 1996. Kinetic properties of glutamate receptor channels in cultured embryonic Drosophila myotubes. Japan J. Physiol. 46, 249-264.

Chang, Y., Weiss, D.S., 2002. Site-specific fluorescence reveals distinct structural changes with GABA receptor activation and antagonism. Nature Neurosci. 5(11), 1163-1168.

Chittajallu, R., Vignes, M., Dev, K.K., Barnes, J.M., Collingridge, G.L., Henley, J.M., 1996. Wingless inactivates glycogen synthase kinase-3 via an intracellular signalling pathway which involves a protein kinase C. EMBO J. 15(17), 45264536.

Cooper, R.L., Marin, L., Atwood, H.L., 1995a. Synaptic differentiation of a single motor neuron: conjoint definition of transmitter release, presynaptic calcium signals, and ultrastructure. J. Neurosci. 15(6), 4209-4222.

Cooper, R.L., Stewart, B.A., Wojtowicz, J.M., Wang, S., Atwood HL., 1995b. Quantal measurement and analysis methods compared for crayfish and Drosophila neuromuscular junctions and rat hippocampus. J. Neurosci. Meth. $61,67-78$.

Cull-Candy, S.G., Parker, I., 1983. Experimental approaches used to examine single glutamate-receptor ion channels in locust muscle fibers. Single-Channel Recording. (ed. by B. Sakmann and E. Neher), pp. 389-400. Plenum Press, New York.

Curutchet, P., Bochet, P., Carvalho, L., Prado, de, Lambolez, B., Stinnakre, J., Rossier, J., 1992. In the GluR1 glutamate receptor subunit a glutamine to histidine point mutation suppresses inward rectification but not calcium permeability. Biochem. Biophys. Res. Commun. 182, 1089-1093.

Dasari, S., Cooper, R.L., 2004. Modulation of sensory to motor circuits by serotonin, octopamine, and dopamine in semi-intact Drosophila larva. Neurosci. Res. 48, 221-227.

Delgado, R., Barla, R., Latorre, R., Labarca, P., 1989. L-glutamate activates excitatory and inhibitory channels in Drosophila larval muscle. FEBS Letters 243, 337-342.

DiAntonio, A., Petersen, S.A., Heckmann, M., Goodman, C.S., 1999. Glutamate receptor expression regulates quantal size and quantal content at the Drosophila neuromuscular junction. J. Neurosci. 19(8), 3023--3032. 
Dingledine, R., Borges, K., Bowie, D., Traynelis, S.F., 1999. The glutamate receptor ion channels. Pharmacol. Rev. 51(1), 7-61.

Dudel, J., Franke, C., Hatt, H., 1992. Rapid activation and desensitization of transmitter-liganded receptor channels by pulses of agonists. Ion Channels (Ed by T. Narahashi) 3rd ed., pp. 207-260. Plenum Press, New York.

Egebjerg, J., Bettler, B., Hermans-Borgmeyer, I., Heinemann, S., 1991. Cloning of a cDNA for a glutamate receptor subunit activated by kainate but not AMPA. Nature 351, 745-748.

Eberle, A.N., Bódi, J., Orosz, G., Süli-Vargha, H., Jäggin, V., Zumsteg, U., 2001. Antagonist and agonist activities of the mouse agouti protein fragment (91-131) at the melanocortin-1 receptor. J. Recept. Signal Transduct Res. 21(1), 25-45.

Featherstone, D.E., Rushton, E., Rohrbough, J., Liebl, F., Karr, J., Sheng, Q., Rodesch, C.K., Broadie, K., 2005. An essential Drosophila glutamate receptor subunit that functions in both central neuropil and neuromuscular junction. J. Neurosci. 25(12), 3199-3208.

Frerking, M., Schmitz, D., Zhou ,Q., Johansen, J., Nicoll, R.A., 2001. Kainate receptors depress excitatory synaptic transmission at CA3-->CA1 synapses in the hippocampus via a direct presynaptic action. J. Neurosci. 21(9), 2958-2966.

Grabauskas, G., Lancaster, B., O'Connor, V., Wheal, H.V., 2007. Protein kinase signalling requirements for metabotropic actions of kainate receptors. J. Physiol. 579, 363-373.

Gration, K.A., Lambert, J.J., Ramsey, R.L., Rand, R.P., Usherwood, P.N., 1981. Agonist potency determination by patch clamp analysis of single glutamate receptors. Brain Res. 230, 400-405.

Guerrero, G., Reiff, D.F., Agarwal, G., Ball, R.W., Borst, A., Goodman, C.S., Isacoff, E.Y., 2005. Heterogeneity in synaptic transmission along a Drosophila larval motor axon. Nature Neuroscience 8, 1188 - 1196.

Harrison, D.A., Cooper, R.L., 2003. Characterization of development, behavior, and neuromuscular physiology in the phorid fly: Megaselia scalaris. Comp. Biochem. Physiol A. 136, 427-439.

He, P., Southard, R.C., Whiteheart, S.W., Cooper, R.L., 1999. Role of alphaSNAP in promoting efficient neurotransmission at the crayfish neuromuscular junction. J. Neurophysiol. 82, 3406-3416. 
Heckmann, M., Dudel, J., 1997. Desensitization and resensitization kinetics of glutamate receptor channels from Drosophila larval muscle. Biophys J. 72(5), 2160-2169.

Hegarty, D.M., Mitchell, J.L., Swanson, K.C., Aicher, S.A., 2007. Kainate receptors are primarily postsynaptic to SP-containing axon terminals in the trigeminal dorsal horn. Brain Res. 1184, 149-159.

Hollman, M., Hainemann, S., 1994. Cloned glutamate receptors. Annu. Rev. Neurosci. 17, 31-108.

Holscher, C., Anwyl, R., Rowan, M. J., 1997. Activation of group-II metabotropic glutamate receptors blocks induction of long term potentiation and depotentiation in area CA1 of the rat in vivo. Eur. J. Pharmacol. 322, 155-163.

Hruby, V.J., Agnes, R.S., Davis, P., Ma, S.W., Lee, Y.S., Vanderah, T.W., Lai, J., Porreca, F., 2003. Design of novel peptide ligands which have opioid agonist activity and CCK antagonist activity for the treatment of pain. Life Sci. 73(6), 699704.

Jan, L.Y., Jan, Y.N., 1976. Properties of the larval neuromuscular junction in Drosophila melanogaster. J. Physiol. 262, 189-214.

Kamiya, H., Ozawa, S., 1998. Kainate receptor-mediated inhibition of presynaptic $\mathrm{Ca} 2+$ influx and EPSP in area CA1 of the rat hippocampus. J. Physiol. 509, 833845.

Kamiya, H., Ozawa, S., 2000. Kainate receptor-mediated presynaptic inhibition at the mouse hippocampal mossy fibre synapse. J. Physiol. 523, 653-665.

Kamiya, H., Ozawa, S., Manabe, T., 2002. Kainate receptor-dependent shortterm plasticity of presynaptic $\mathrm{Ca} 2+$ influx at the hippocampal mossy fiber synapses. J. Neurosci. 22, 9237-9243.

Kerchner, G.A., Wilding, T.J., Li, P., Zhuo, M., Huettner, J.E., 2001. Presynaptic kainate receptors regulate spinal sensory transmission. J. Neurosci. 21(1), 59-66.

Kurdyak, P., Atwood, H.L., Stewart, B.A., Wu, C.F., 1994. Differential physiology and morphology of motor axons to ventral longitudinal muscle in larval Drosophila. J. Comp. Neurol. 350, 463-472.

Lerma, J., 2003. Roles and rules of kainate receptors in synaptic transmission. Nat. Rev. Neurosci. 4(6), 481-495.

Lee, C.J., Kong, H., Manzini, M.C., Albuquerque, C., Chao, M.V., MacDermott, A.B., 2001. Kainate receptors expressed by a subpopulation of developing 
nociceptors rapidly switch from high to low $\mathrm{Ca} 2+$ permeability. J. Neurosci. 21(13), 4572-4581.

Li, H., Cooper, R.L., 2001. Maintaining synaptic efficacy at the neuromuscular junction in Drosophila larva during normal development and prolonged life with the ecdysoneless mutant. Neurosci. 106, 193-200.

Li, H., Peng, X., Cooper, R.L., 2002. Development of Drosophila larval neuromuscular junctions: Maintaining synaptic strength. Neurosci. 115, 505-513.

Liebl, F.L., Featherstone, D.E., 2005. Genes involved in Drosophila glutamate receptor expression and localization. BMC Neurosci. 6, 44.

Littleton, J.T., Ganetzky, B., 2000. Ion channels and synaptic organization: analysis of the Drosophila genome. Neuron 26, 35-43.

Lipsky, R.H., Goldman, D., 2003. Genomics and variation of ionotropic glutamate receptors. Ann. NY Acad. Sci. 1003, 22-35.

Logsdon, S., Johnstone, A.F.M., Viele, K., Cooper, R.L., 2006. The regulation of synaptic vesicles pools within motor nerve terminals during short-term facilitation and neuromodulation. Appl. Physiol. 100(2), 662-671.

Marrus, S.B., Portman, S.L., Allen, M.J., Moffat, K.G., DiAntonio, A., 2004. Differential localization of glutamate receptor subunits at the Drosophila neuromuscular junction. J. Neurosci. 24, 1406-1415.

Mayer, M.L., 2005. Glutamate receptor ion channels. Curr. Opin. Neurobiol. 15(3), 282-289.

McLarnon, J.G., Quastel, D.M., 1988. A voltage clamp study of the glutamate responsive neuromuscular junction in Drosophila melanogaster. Canadian J. Physiol. Pharmacol. 66, 321-327.

Nicoll, R.A., Mellor, J., Frerking, M., Schmitz, D., 2000. Kainate receptors and synaptic plasticity. Nature. 406(6799), 957.

Park, Y., Jo, J., Isaac, J.T., Cho, K., 2006. Long-term depression of kainate receptor-mediated synaptic transmission. Neuron 49(1), 95-106.

Patlak, J.B., Gration, K.A., Usherwood, P.N., 1979. Single glutamate-activated channels in locust muscle. Nature 5705, 643-645.

Pauly, T., Schlicksupp, A., Neugebauer, R., Kuhse, J., 2005. Synaptic targeting of $\mathrm{N}$-methyl-D-aspartate receptor splice variants is regulated differentially by receptor activity. Neurosci. 131(1), 99-111. 
Pawlu, C., DiAntonio, A., Heckmann, M., 2004. Postfusional control of quantal current shape. Neuron 42(4), 607-618.

Pinheiro, P.S., Mulle, C., 2008. Presynaptic glutamate receptors: physiological functions and mechanisms of action. Nat. Rev. Neurosci. 9, 423-436.

Qin, G., Schwarz, T., Kittel, R.J., Schmid, A., Rasse, T.M., Kappei, D., Ponimaskin, E., Heckmann, M., Sigrist, S.J., 2005. Four different subunits are essential for expressing the synaptic glutamate receptor at neuromuscular junctions of Drosophila. J. Neurosci. 25(12), 3209-3218.

Rasse, T.M., Fouquet, W., Schmid, A., Kittel, R.J., Mertel, S., Sigrist, C.B., Schmidt, M., Guzman, A., Merino, C., Qin, G., Quentin, C., Madeo, F.F., Heckmann, M., Sigrist, S.J., 2005. Glutamate receptor dynamics organizing synapse formation in vivo. Nature Neurosci. 8(7), 898-905.

Rozas, J.L., Paternain, A.V., Lerma, J., 2003. Noncanonical signaling by ionotropic kainate receptors. Neuron 39, 543-553

Ruffner, M.E., Cromarty, S.I., Cooper, R.L., 1999. Depression of synaptic efficacy in Drosophila neuromuscular junctions by the molting hormone (20Hydroxyecdysone). J. Neurophysiol. 81, 788-794.

Schoepp, D.D., 2002. Metabotropic glutamate receptors. Pharmacol. Biochem. Behav. 73(2), 285-286.

Schoepp, D., Bockaert, J., Sladeczek, F., 1990. Pharmacological and functional characteristics of metabotropic excitatory amino acid receptors. Trends Pharmacol. Sci. 11, 508-515.

Schramm, M., Dudel, J., 1997. Metabotropic glutamate autoreceptors on nerve terminals on crayfish muscle depress or facilitate release. Neurosci. Lett., 234, $31-34$.

Schuster, C.M., Ultsch, A., Schloss, P., Cox, J.A., Schmitt, B., Betz, H., 1991. Molecular cloning of an invertebrate glutamate receptor subunit expressed in Drosophila muscle. Science 254(5028), 112-114.

Seeburg, P.H., 1993. The TINS/TiPS Lecture. The molecular biology of mammalian glutamate receptor channels. Trends Neurosci. 16(9), 359-365.

Shinozaki, H., Ishida, M., 1981. Quisqualate action on the crayfish neuromuscular junction. J. Pharmacobiodynamics 4, 42-48. 
Shinozaki, H., Shibuya, I., 1974. A new potent excitant, quisqualic acid: effects on crayfish neuromuscular junction. Neuropharmacol. 13, 665-672.

Sigrist, S.J., Thiel, P.R., Reiff, D.F., Schuster, C.M., 2002. The postsynaptic glutamate receptor subunit DGluR-IIA mediates long-term plasticity in Drosophila. Neurosci. 22(17), 7362-7372.

Sigrist, S.J., Reiff, D.F., Thiel, P.R., Steinert, J.R., Schuster, C.M., 2003. Experience-dependent strengthening of Drosophila neuromuscular junctions. J. Neurosci. 23(16), 6546-6556.

Sparks, G.M., Dasari, S., Cooper, R.L., 2004. Actions of MDMA at glutamatergic neuromuscular junctions. Neurosci. Res. 48(4), 431-438.

Stewart, B.A., Atwood, H.L., Renger, J.J., Wang, J., Wu, C.F., 1994. Improved stability of Drosophila larval neuromuscular preparation in haemolymph-like physiological solutions. J. Comp. Physiol. A 175, 179-191.

Stewart, B.A., Schuster, C.M., Goodman, C.S., Atwood, H.L., 1996. Homeostasis of synaptic transmission in Drosophila with genetically altered nerve terminal morphology. J. Neurosci. 16(12), 3877-3886.

Sun, Y., Olson, R., Horning, M., Armstrong, N., Mayer, M.L., Gouaux, E., 2002. Mechanism of glutamate receptor desensitization. Nature 417, 245-253.

Thiagarajan, T.C., Lindskog, M., Tsien, R.W., 2005. Adaptation to synaptic inactivity in hippocampal neurons. Neuron 47(5), 725-737.

Tour, O., Parnas, H., Parnas, I., 2000. On the Mechanism of Desensitization in Quisqualate-Type Glutamate Channels. J. Neurophysiol. 84,1-10.

Verdoorn, T.A., Burnashev, N., Monyer, H., Seeburg, P.H., Sakmann, B., 1991. Structural determinants of ion flow through recombinant glutamate receptor channels. Science 252(5013),1715-1718.

Völkner, M., Lenz-Bohme, B., Betz, H., Schmitt, B., 2000. Novel CNS glutamate receptor subunit genes of Drosophila melanogaster. J. Neurochem. 75(5), 17911799.

Zhang, D., Kuromi, H., Kidokoro, Y., 1999. Activation of metabotropic glutamate receptors enhances synaptic transmission at the Drosophila neuromuscular junction. Neuropharmacol. 38, 645-657. 


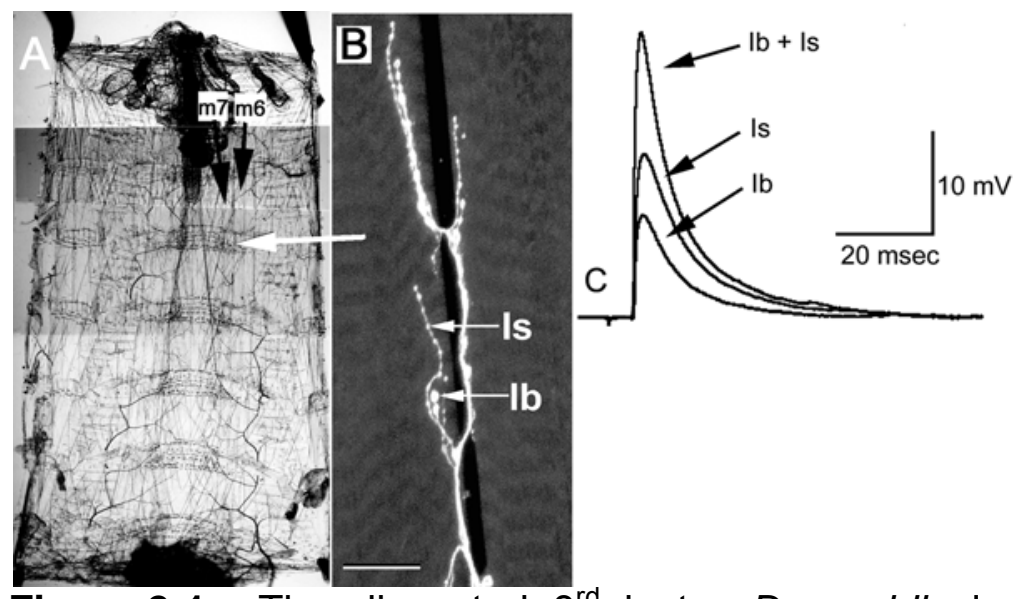

Figure 2-1: The dissected $3^{\text {rd }}$ instar Drosophila larva preparation. (A) The preparation is pinned at the four corners to keep the preparation taut. The ventral abdominal muscle $\mathrm{m} 6$ is used in this study. The segmental nerves are stimulated by placing the nerve into a suction electrode and recruiting the Is and $\mathrm{Ib}$ motor neurons. (B) The terminals of $\mathrm{Ib}$ and Is on $\mathrm{m} 6$ and $\mathrm{m} 7$ are readily observed after treatment with fluorescently tagged anti-HRP antibody (Scale: $50 \mu \mathrm{m})$. (C) Elicited excitatory postsynaptic potentials (EPSPs) in $\mathrm{m} 6$ are measured with an intracellular recording. Representative individual responses from the $\mathrm{Ib}$ and Is motor axons as well as the composite $\mathrm{lb}$ and Is response are shown for a early 3rd instar. 

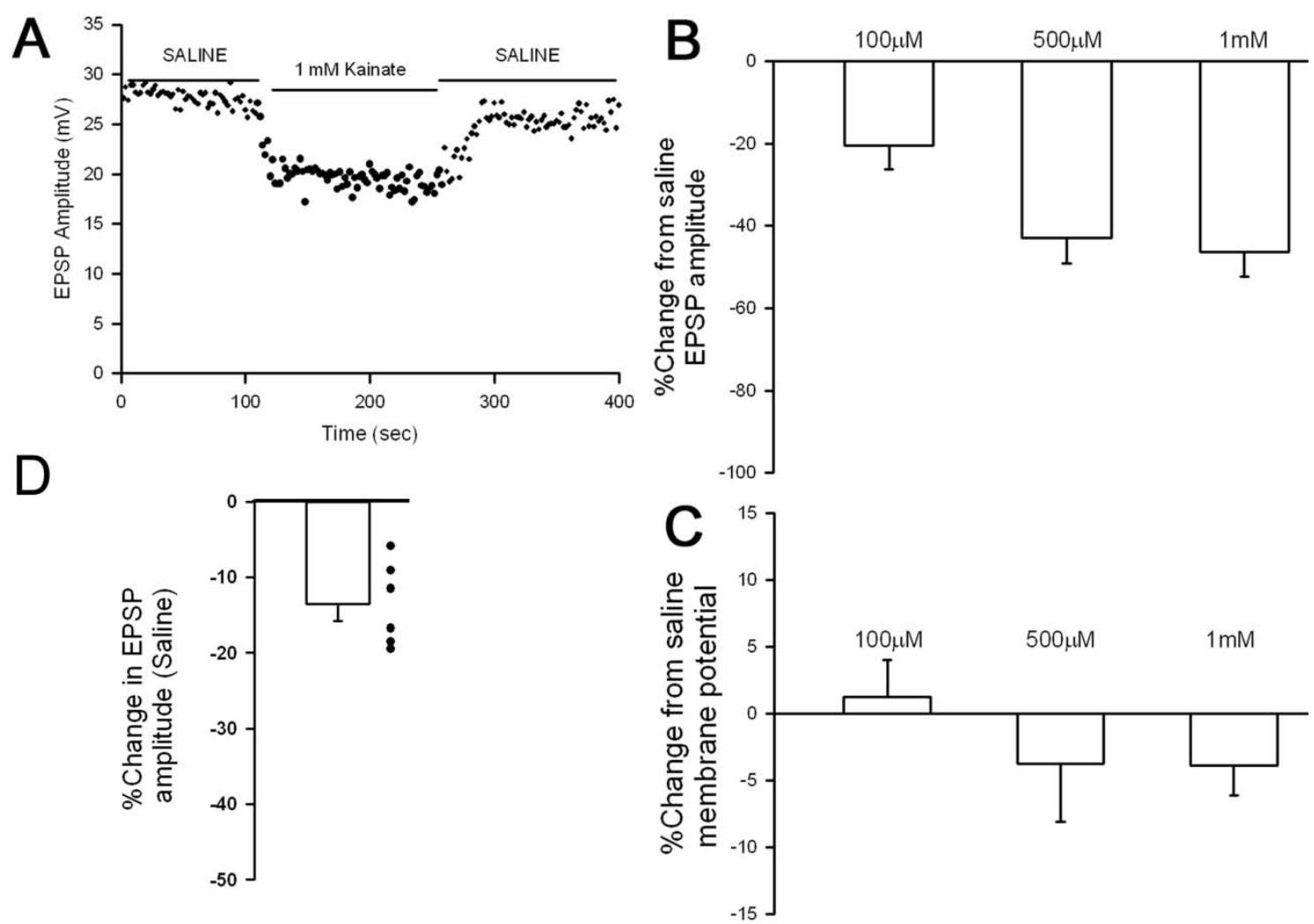

Figure 2-2: The effect of kainate on the evoked EPSP amplitudes. (A) Application of $1 \mathrm{mM}$ kainate reduces the EPSP rapidly and the effect can be partially washed out. (B) The dose-response effect in damping the EPSP amplitude. For all concentrations of kainate the EPSP amplitudes are reduced. (C) The dose-response effects on the resting membrane potential for $\mathrm{m} 6$. The resting membrane potentials do not appear to be changed. With the maintaining resting membrane potential, reduced EPSP amplitudes are induced by the antagonistic action of kainate. (D) Sham control for exchanging the saline bath and time revealed a $13.5 \%$ reduction in the EPSP amplitude. When compared to sham control, both 500 $\mu \mathrm{M}$ and $1 \mathrm{mM}$ still show significantly reduced EPSPs (around $46 \%$ decrease) 


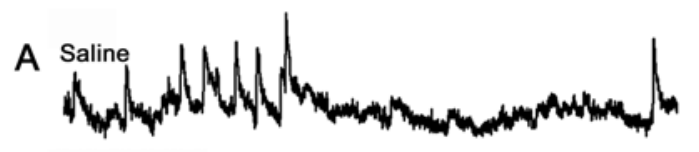

Domoic Acid (1 mM)
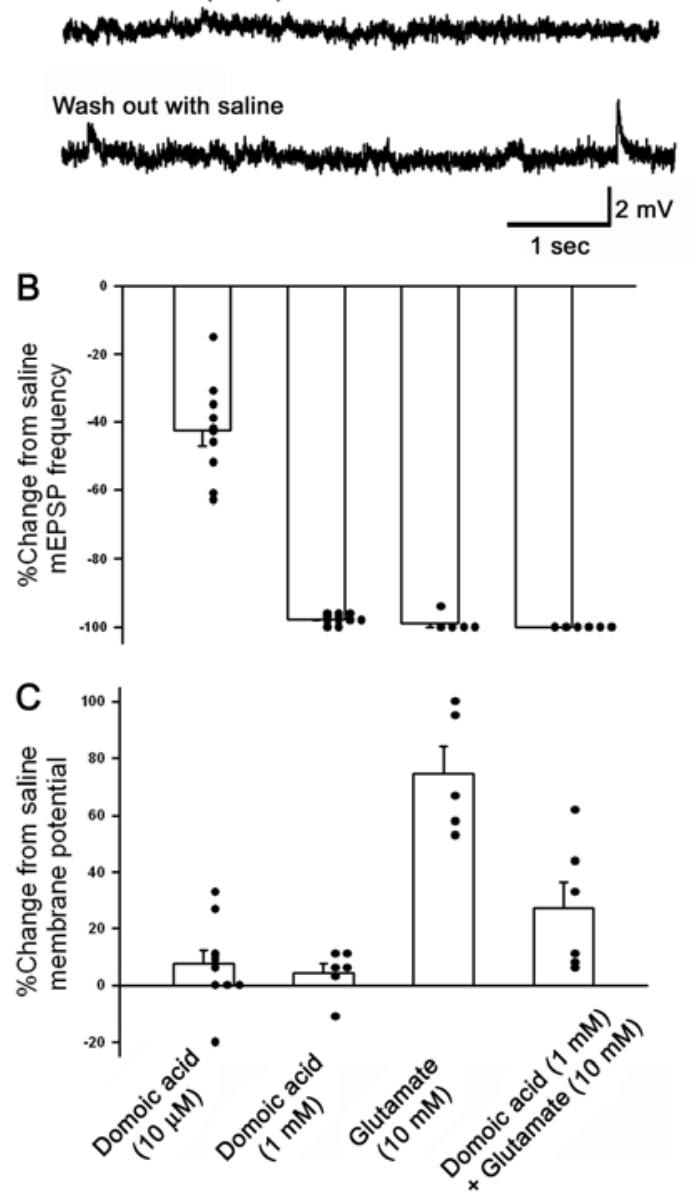

Figure 2-3: Effects of domoic acid (agonist for AMPA and kainate receptors) at the NMJ. Domoic acid is a potent kainate receptor agonist. (A) Spontaneous or mEPSPs (miniature EPSPs) are reduced in amplitude upon exposure to $1 \mathrm{mM}$ of domoic acid. With washing out the domoic acid, the spontaneous responses gradually come up again. (B) The frequency of mEPSPs is reduced by domoic acid in a dose-dependent manner. Glutamate mimics the effect. (C) Domoic acid did not produce a consistent effect on the resting membrane potential; however glutamate produced a drastic depolarization fitting for agonistic action. Domoic acid attenuates the effect of glutamate on membrane depolarization. So, domoic acid appears to be blocking the glutamate action on the muscle. 


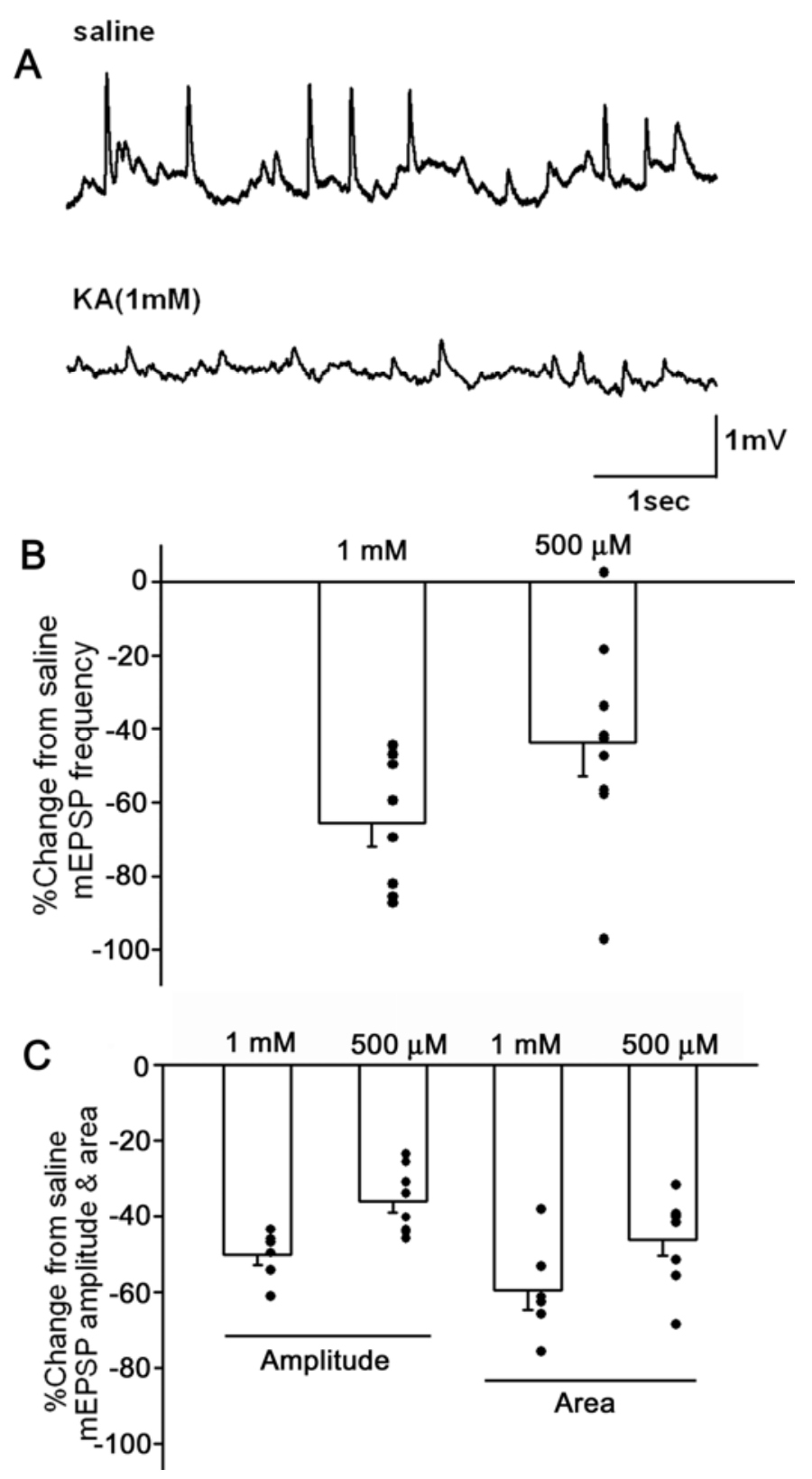

Figure 2-4: Effects of kainate (1 and $0.5 \mathrm{mM}$ ) on mEPSPs properties. (A) Spontaneous or mEPSPs (miniature EPSPs) are reduced in amplitude upon exposure. (B) The frequency of mEPSPs is reduced by domoic acid in a dose-dependent manner, as well as the (C) amplitude and area of the mEPSPs. Kainate seems to be acting presynaptically as well as postsynaptically. However, the problem of this measuring is that the mEPSPs can be buried within the noise as the amplitude of mEPSPs are reduced. 

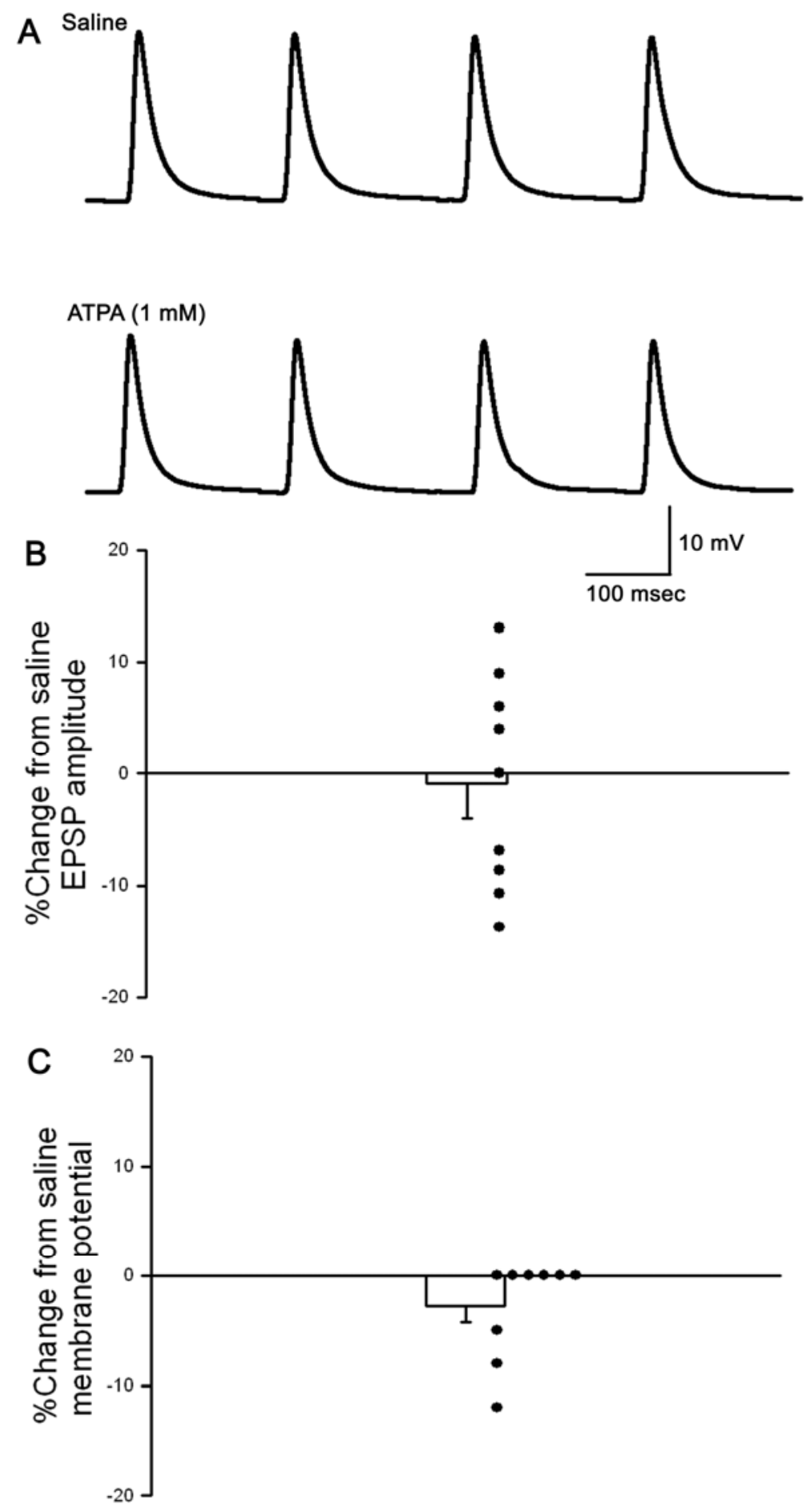

Figure 2-5: Effects of ATPA ( $1 \mathrm{mM}$; a selective agonist of vertebrate GluR5, kainate receptors) on the amplitude of evoked EPSPs (A), (B) as well as the resting membrane potential of the muscle (C). ATPA does not appear to alter the EPSP amplitude or the resting membrane potential. 

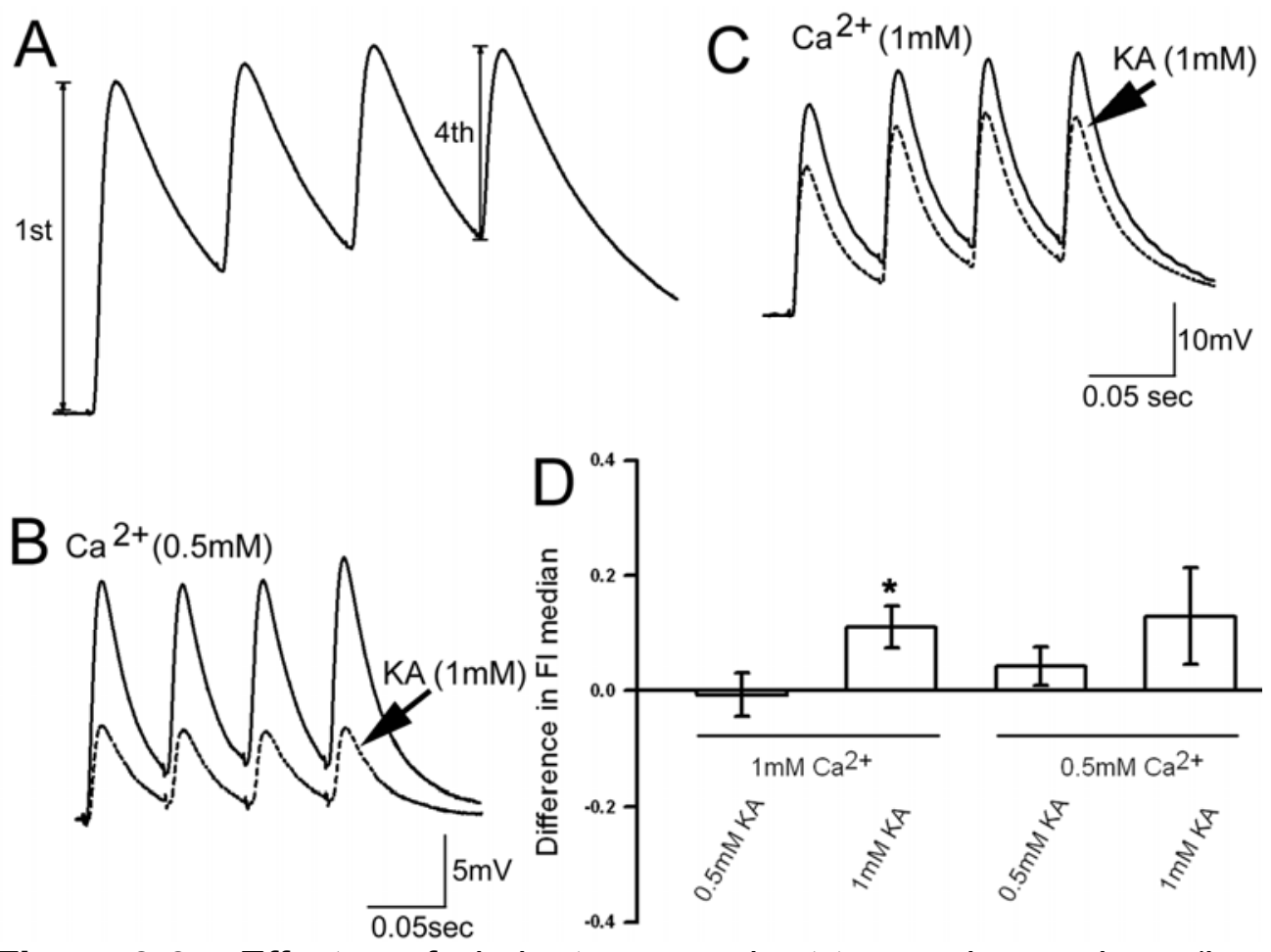

Figure 2-6: Effects of kainate on short-term depression (i.e., negative facilitation) with varied $\left[\mathrm{Ca}^{2+}\right]_{0}$. (A) A four pulse stimulus train delivered at $20 \mathrm{~Hz}$ results in an initial large EPSP followed by depressed responses. The procedure for measuring EPSP amplitudes is indicated. Facilitation index $(\mathrm{FI})$ is then calculated from these measures. (B) A typical response for the effect of kainate $(\mathrm{KA} ; 1 \mathrm{mM})$ on the pulse train in the presence of $0.5 \mathrm{mM}$ $\left[\mathrm{Ca}^{2+}\right]_{0}$. (C) A typical response for the effect of KA (1 mM) on the pulse train in the presence of $1 \mathrm{mM}\left[\mathrm{Ca}^{2+}\right]_{\mathrm{o}}$. (D) The difference in facilitation index $(\mathrm{FI})$ is shown for both $1 \mathrm{mM}$ and $0.5 \mathrm{mM} \mathrm{KA}$ with either $1 \mathrm{mM}$ or $0.5 \mathrm{mM}\left[\mathrm{Ca}^{2+}\right]_{\mathrm{o}}$. The differences in the median values are obtained from the difference in $\mathrm{FI}$ saline to exposure of KA within each preparation. ( $n=5$ for each paradigm; * $p<0.05$ Wilcoxon Rank Sum Test for the effect of KA as compared to saline). 


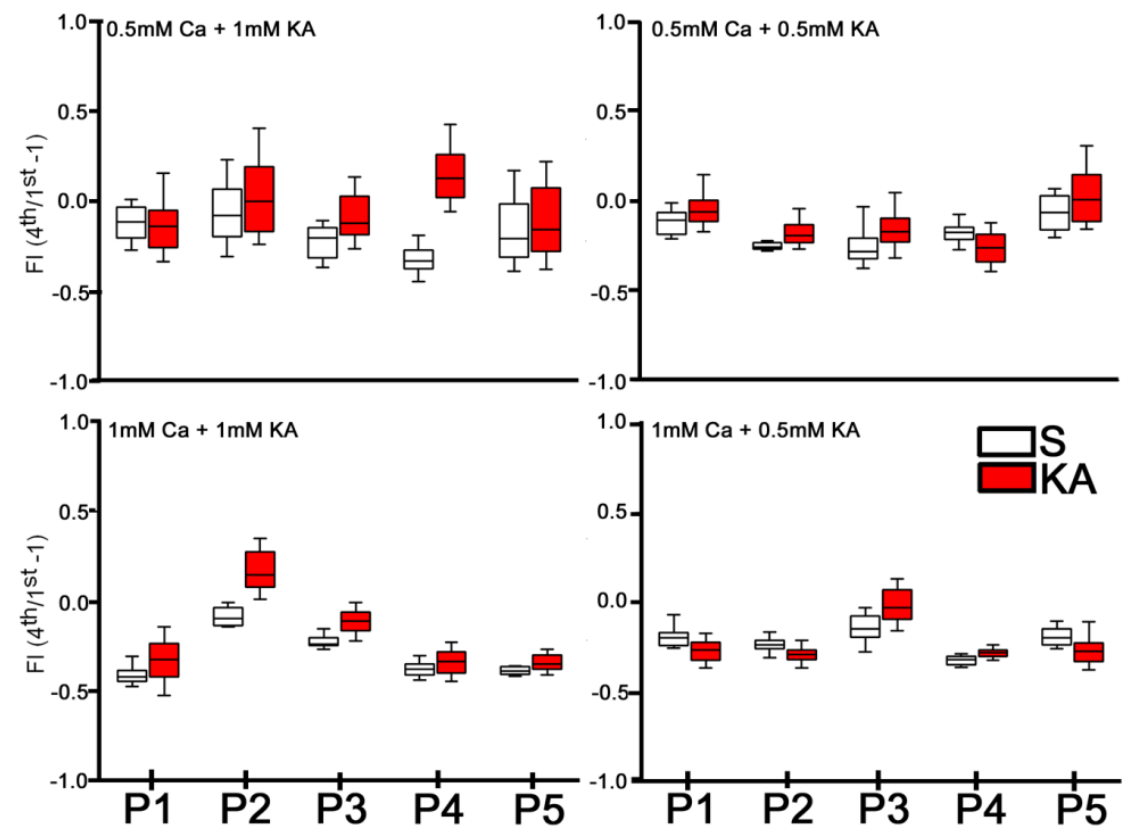

Figure 2-7: Effects of kainate (KA) and calcium on FI. Box and whisker plots for $\mathrm{FI}$ in various conditions are shown as before and after exposure to kainate, for each of the five preparations in the four experimental conditions. The plots show the median, $25^{\text {th }}, 75^{\text {th }}$ percentiles and range. In almost all preparations, median values of $\mathrm{FI}$ index seem to be the same before and after exposure to kainate. So, kainate does not appear to have a presynaptic action. With $1 \mathrm{mM} \mathrm{Ca}^{2+}$ and $1 \mathrm{mM}$ kainate, both median and variation of $\mathrm{FI}$ index are increased in five out of five preparations after exposure to kainate. However, after changing the [kainate] into $0.5 \mathrm{mM}$, neither median nor variation of facilitation index shows a consistent increase in values. So, kainate seems to be acting as a competitive antagonist. 


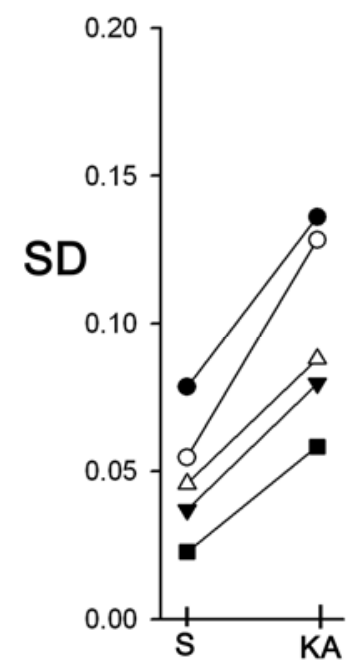

Figure 2-8: The standard deviation (SD) for each preparation before and during exposure to kainate $(1 \mathrm{mM})$ with $1 \mathrm{mM}\left[\mathrm{Ca}^{2+}\right]_{\mathrm{o}}$ produced a consistent trend in increasing in the variability among the data sets while exposed to kainate (KA) as compared the saline (S) baseline $(p<0.05$, Wilcoxon Rank Sum Test; also $p<0.5$ Student's $t$-test). 

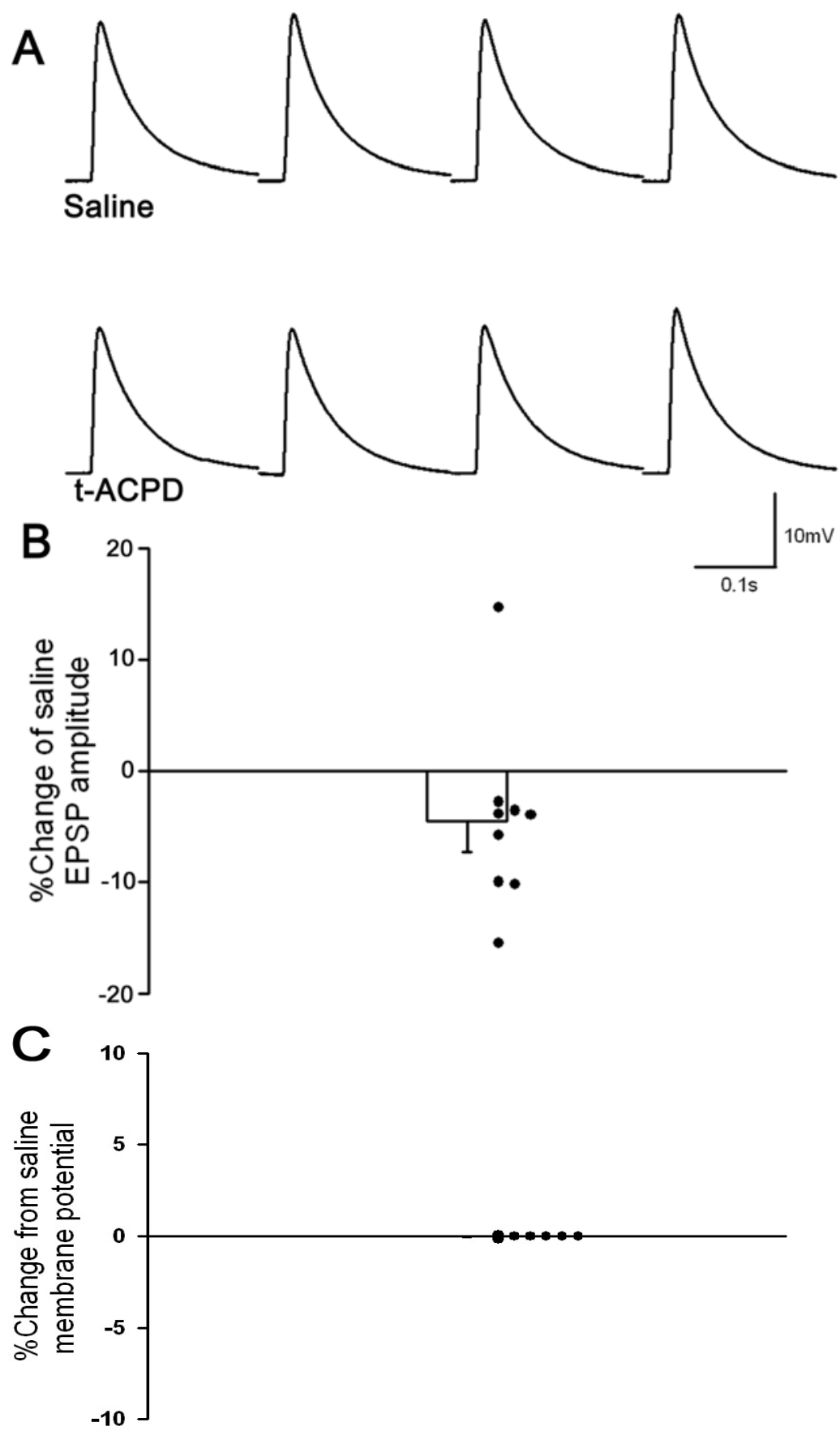

Figure 2-9: Action of t-ACPD on evoked EPSPs and resting membrane potential. (A) Evoked EPSPs in saline and during exposures to tACPD $(50 \mu \mathrm{M})$. (B) The mean percent change in the amplitude of the evoked EPSPs with exposure to t-ACPD. (C) The mean percent change in the resting membrane potential with exposure to t-ACPD. $\mathrm{t}-\mathrm{ACPD}$ does not appear to have an action on the postsynaptic muscle or the glutamate receptors. 
GluR5

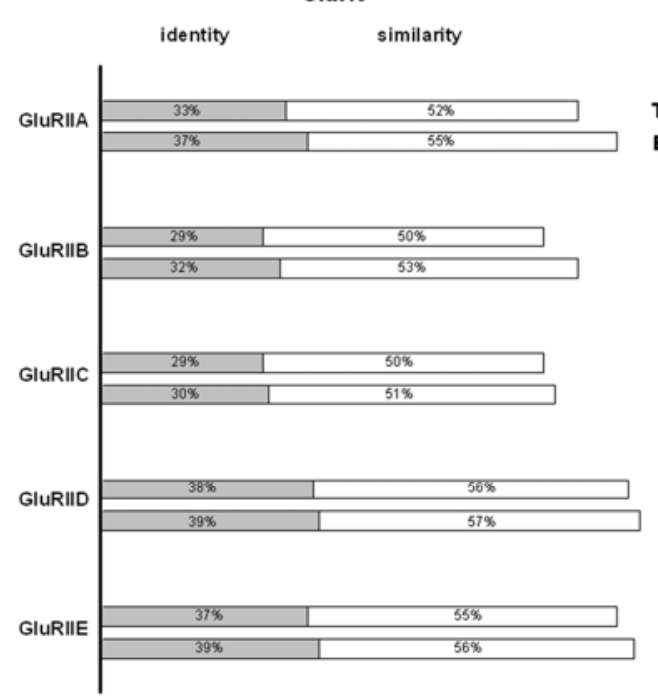

GluR6
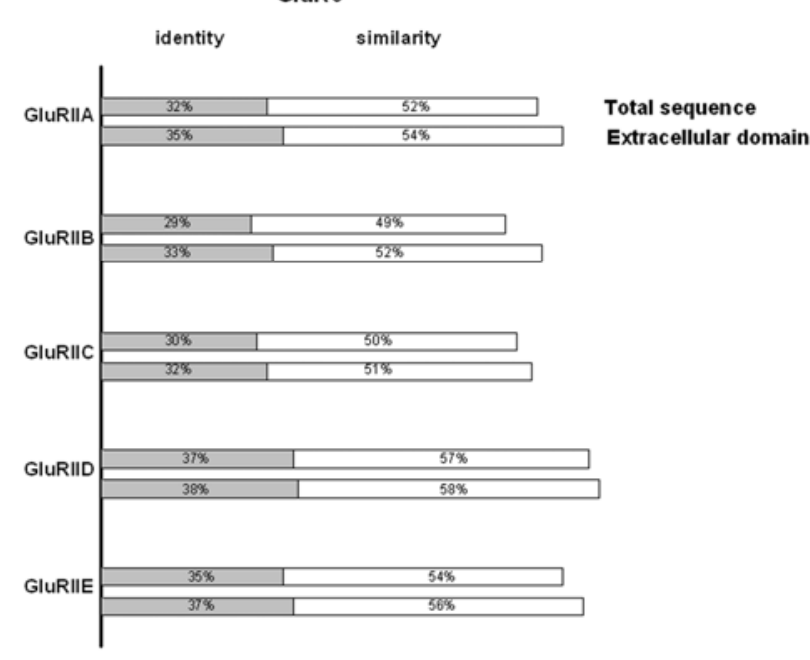

GluR7

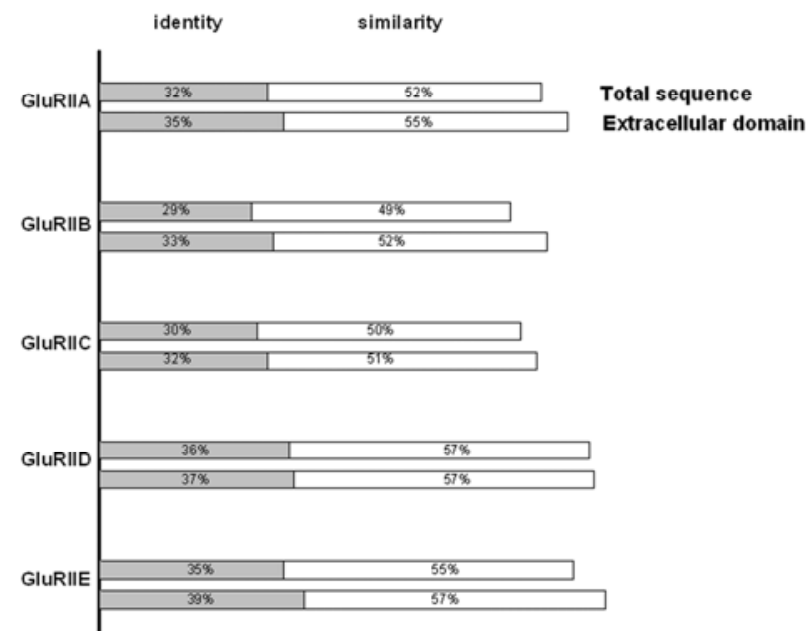

KA2
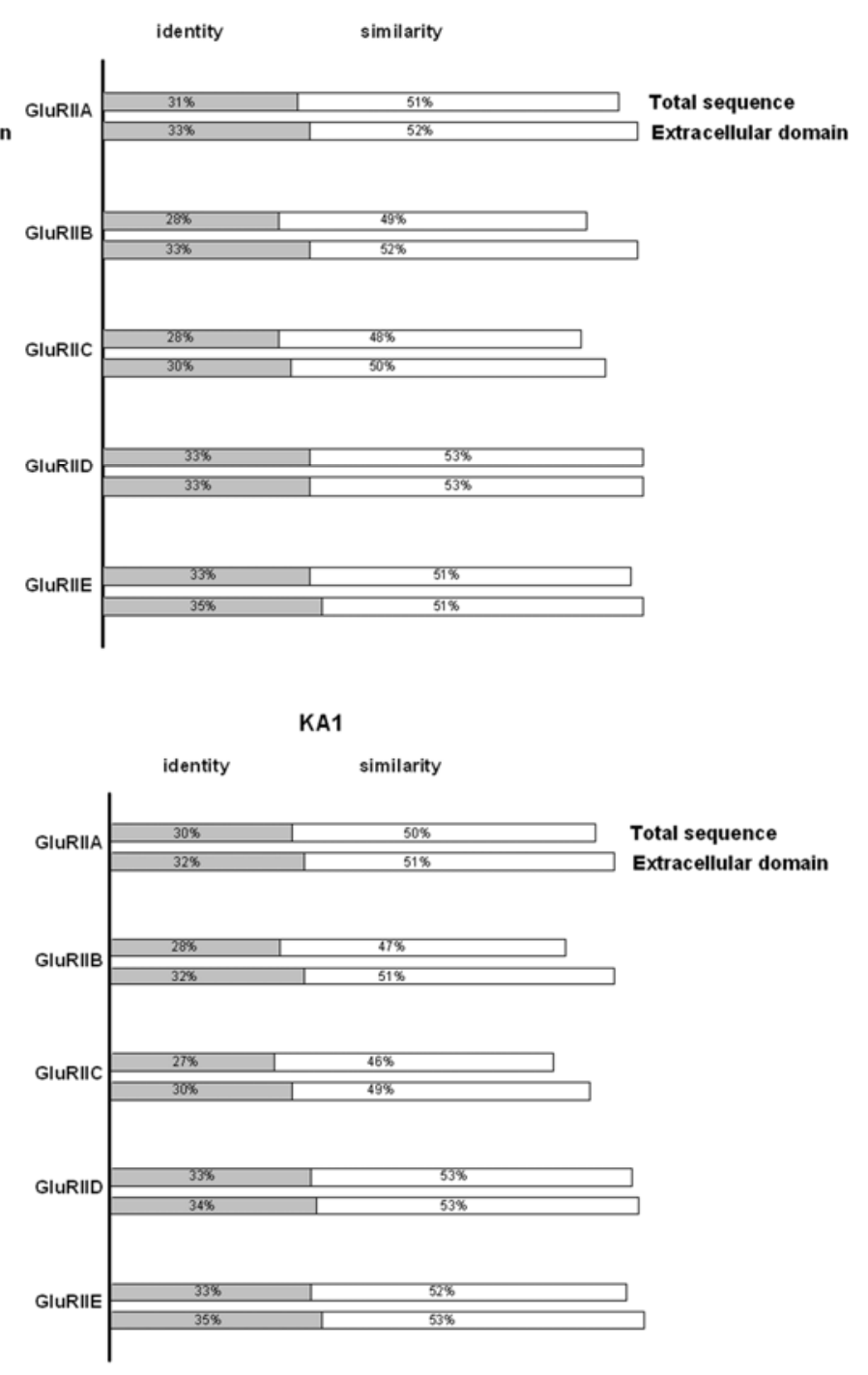
Figure 2-10: Sequence comparison of five postsynaptic receptor subunits identified from Drosophila muscle mRNA to the five known human kainate receptor subunits. Each two sequences were compared by using a blastP alignment. The top bar is a comparison for the total protein sequence. The bottom bar is a comparison of the putative extracellular domains among the proteins. The gray area is the percentage of identical sequence and the white are is the percent in similarity based on the blastP (NCBI) algorithm. The five human receptor subunits used are: (A) GluR5; (B) GluR6; (C) GluR7; (D) $\mathrm{KA} 1$; and (E) KA2.

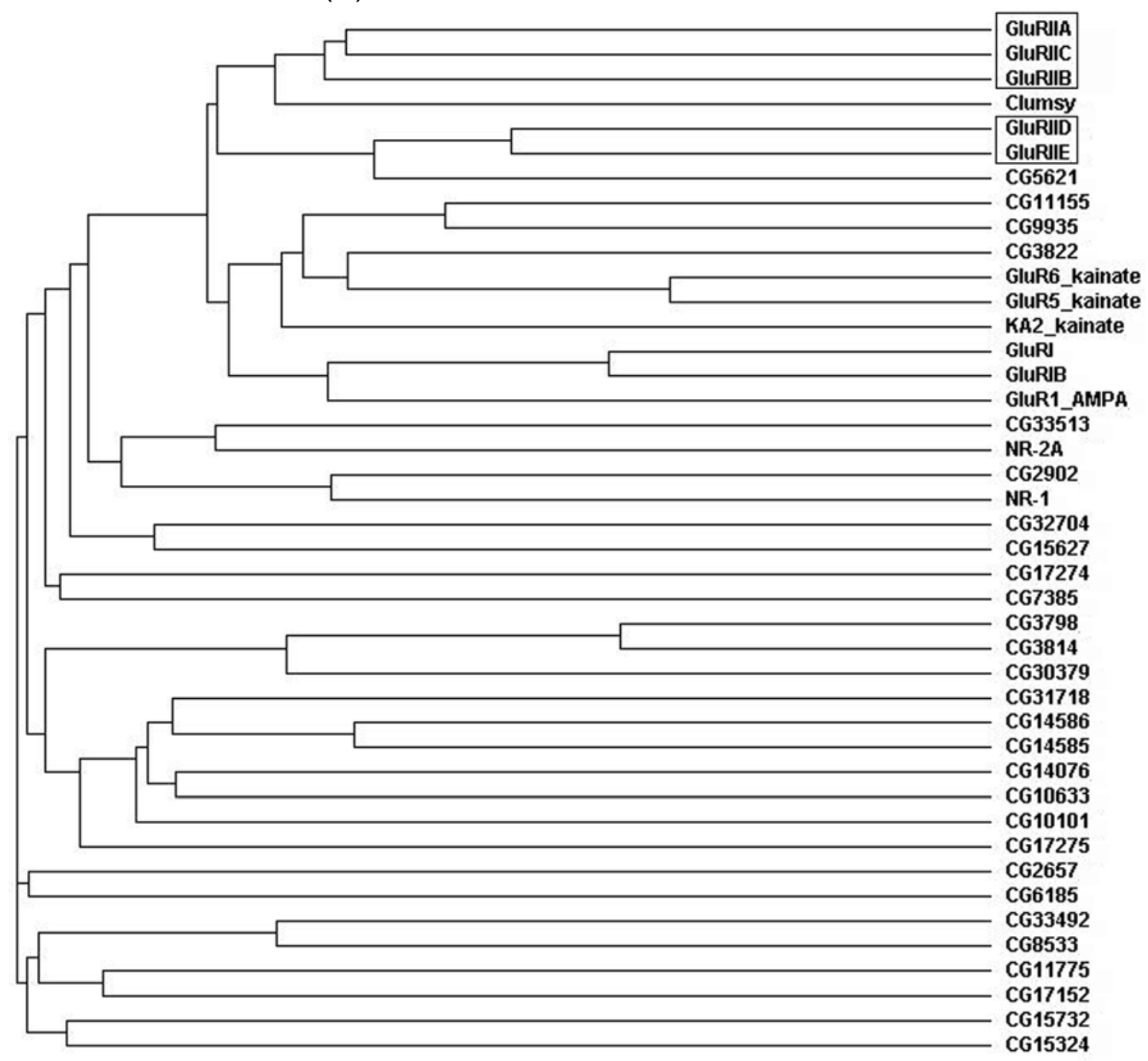

Figure 2-11: Phylogeny tree comparing all known putative 36 ionotropic glutamate receptor subunits in the Drosophila genome with human glutamate receptors (kainate receptor subunits GluR5, GluR6 and KA2; AMPA receptor subunit GluR1; NMDA receptor subunits NR-1 and NR-2). The comparison was performed by ClustalW2 (EMBL$\mathrm{EBI})$. 
Author's Name - Junyoung Lee

Birthplace - Jeju, South Korea

Birthdate - January 9, 1982

\section{Education}

Bachelor of Science in Chemistry

Korea Military Academy

Febuary - 2004

Military Commission

Second Lieutenant: 2004 - 2005

First Lieutenant: 2005 - 2007

Captain: 2007 - present

\section{$\underline{\text { Publications }}$}

Lee, J.-Y., Bhatt, D., Bhatt, D., Chung, W.-Y., and Cooper, R.L. (2009) Furthering pharmacological and physiological assessment of the glutamatergic receptors at the Drosophila neuromuscular junction. (In Review).

\section{Abstracts}

1. Lee, J.Y., Chung, W.Y., Logsdon, S., Johnstone, A. F. M., Cooper, R.L. (2008) The regulation of synaptic vesicle pools within motor nerve 
terminals. Society for Integrative and Comparative Biology. Annual meeting. January 2-6, 2008, San Antonio, TX.

2. Lee, J.Y., Chung, W.Y., Logsdon, S., Johnstone, A. F. M., Cooper, R.L. (2008) The regulation of synaptic vesicle pools within motor nerve terminals. Annual meeting of the BlueGrass Chapter of the Society for Neuroscience. March12, 2008. Univ. of KY.

3. Lee, J.-Y., Bhatt, D., Bhatt, D., Chung, W.-Y., Lee, N.-T. and Cooper, R.L. (2008) Pre- \& Post-synaptic Actions of Kainate: Negative Feedback at Glutamate-ergic Nerve Terminals. Annual meeting of Society for Neuroscience. Washington, DC.

4. Lee, J.-Y., Bhatt, D., Bhatt, D., Chung, W.-Y., Lee, N.-T. and Cooper, R.L. (2008) Pre- \& Post-synaptic Actions of Kainate: Negative Feedback at Glutamatergic Nerve Terminals. Kentucky Academy of Sciences. Annual meeting. November 1, 2008, Lexington, KY (Univ. of KY campus).

5. Lee, J.-Y., Bhatt, D., Bhatt, D., Chung, W.-Y., Lee, N.-T. and Cooper, R.L. (2009) Pre- \& Post-synaptic Actions of Kainate: Negative Feedback at Glutamate-ergic Nerve Terminals. Society for Integrative and Comparative Biology. Annual meeting. January 3-7, 2008, Boston, MA.

\section{Outreaching}

Judging local science fairs

1. 2008. Judged at Central KY Regional Science and Engineering Fair. Held at the Univ of $\mathrm{KY}$

2. 2008 \& 2009 Judged at Glendover elementary school.

3. $2008 \& 2009$ Judged at Morton middle school. 